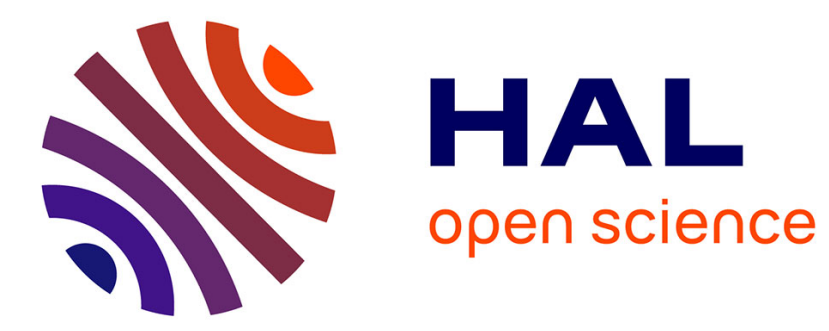

\title{
Granular and particle-laden flows: from laboratory experiments to field observations
}

Renaud Delannay, Alexandre Valance, Anne Mangeney, Olivier Roche, Patrick Richard

\section{- To cite this version:}

Renaud Delannay, Alexandre Valance, Anne Mangeney, Olivier Roche, Patrick Richard. Granular and particle-laden flows: from laboratory experiments to field observations. Journal of Physics D: Applied Physics, 2017, 50 (5), pp.40. 10.1088/1361-6463/50/5/053001 . hal-01481019

HAL Id: hal-01481019 https://hal-univ-rennes1.archives-ouvertes.fr/hal-01481019

Submitted on 17 Oct 2017

HAL is a multi-disciplinary open access archive for the deposit and dissemination of scientific research documents, whether they are published or not. The documents may come from teaching and research institutions in France or abroad, or from public or private research centers.
L'archive ouverte pluridisciplinaire HAL, est destinée au dépôt et à la diffusion de documents scientifiques de niveau recherche, publiés ou non, émanant des établissements d'enseignement et de recherche français ou étrangers, des laboratoires publics ou privés. 


\title{
Granular and particle-laden flows: from laboratory experiments to field observations
}

\author{
R Delannay $^{1}$, A Valance ${ }^{1}$, A Mangeney ${ }^{2,3}$, O Roche ${ }^{4}$, P Richard ${ }^{5}$ \\ ${ }^{1}$ Institut de Physique de Rennes, UMR CNRS 6251, Université de Rennes 1, Campus de Beaulieu \\ Bâtiment 11A, 263 av. Général Leclerc, 35042 Rennes CEDEX, France \\ ${ }^{2}$ Université Paris Diderot, Sorbonne Paris Cité, Institut de Physique du Globe de Paris, Seismology \\ group, 1 rue Jussieu, 75005 Paris, France \\ ${ }^{3}$ ANGE Team, CEREMA, INRIA, Lab. J. Louis Lions, Paris, France \\ ${ }^{4}$ Laboratoire Magma et Volcans, Université Blaise Pascal-CNRS-IRD, OPGC, Campus Universitaire les \\ Cézeaux, 6 avenue Blaise Pascal, 63178 Aubière, France \\ France \\ ${ }^{5}$ LUNAM Université, IFSTTAR, MAST, GPEM, site de Nantes, Route de Bouaye, 44344 Bouguenais \\ cedex, France
}

E-mail: renaud.delannay@univ-rennes1.fr

\begin{abstract}
This review article provides an overview of dry granular flows and particle fluid mixtures, including experimental and numerical modeling at the laboratory scale, large scale hydrodynamics approaches and field observations. Over the past ten years, the theoretical and numerical approaches have made such significant progress that they are capable of providing qualitative and quantitative estimates of particle concentration and particle velocity profiles in steady and fully developed particulate flows. The next step which is currently developed is the extension of these approaches to unsteady and inhomogeneous flow configurations relevant to most of geophysical flows. We also emphasize that the up-scaling from laboratory experiments to large scale geophysical flows still poses some theoretical physical challenges. For example, the reduction of the dissipation that is responsible for the unexpected long run-out of large scale granular avalanches is not observed at the laboratory scale and its physical origin is still a matter of debate. However, we believe that the theoretical approaches have reached a mature state and that it is now reasonable to tackle complex particulate flows that incorporate more and more degrees of complexity of natural flows.
\end{abstract}

\section{Introduction}

Geophysical granular and particle laden flows are common phenomena at the surface of the Earth and other planets (Figures 1 and 2). They consist of solid-fluid mixtures driven by gravity and that propagate in an ambient fluid (except on some extraterrestrial planets) over generally complex topographies. Investigating these phenomena is important because they play a significant role in the global sediment cycle and contribute to shape the landscape. Furthermore, gravitational flows and natural phenomena they can generate, such as tsunamis for instance (Kawamura et al. (2014)), represent severe natural hazards for the populations and infrastructures. Determination of their occurrence and magnitude can also help inferring major climate changes as well as the characteristics of triggering mechanisms. Indeed, gravitational flows are closely related to climatic, volcanic, and seismic activity and thus represent proxies for the time change of these activities (Calder et al. (2002), Hibert et al. (2011, 2014a), DeRoin and McNutt (2012)). This is also of paramount importance to infer 
the current or past presence of water on planets such as Mars (Mangold et al. (2010)). Their study is of economic interest as accumulation of deposits in submarine environments may form large hydrocarbon reservoirs while submarine landslides may destroy sea bed infrastructures such as transoceanic communication cables.

Table 1. Main types of geophysical flows and typical ranges of values of most relevant parameters.

\begin{tabular}{|c|c|c|c|c|c|c|c|c|c|}
\hline Flow type & $\begin{array}{c}\text { setting, } \\
\text { ambient fluid }\end{array}$ & $\begin{array}{l}\text { interstitial } \\
\text { fluid }\end{array}$ & $\begin{array}{c}\text { particle } \\
\text { size } \\
(\mathrm{m}) \\
\end{array}$ & $\begin{array}{c}\text { particle } \\
\text { density } \\
\left(\mathrm{kg} \mathrm{m}^{-3}\right)\end{array}$ & $\begin{array}{c}\text { particle } \\
\text { volume } \\
\text { fraction }\end{array}$ & $\begin{array}{c}\text { volume } \\
\left(\mathrm{m}^{3}\right)\end{array}$ & $\begin{array}{c}\text { velocity } \\
\left(\mathrm{m} \mathrm{s}^{-1}\right)\end{array}$ & $\begin{array}{l}\text { thickness } \\
\text { (m) }\end{array}$ & $\begin{array}{c}\text { runout } \\
\text { distance } \\
(\mathbf{k m})\end{array}$ \\
\hline $\begin{array}{l}\text { Subaerial } \\
\text { landslides, } \\
\text { rockfalls, } \\
\text { rock or } \\
\text { debris } \\
\text { avalanches }\end{array}$ & $\begin{array}{l}\text { subaerial, } \\
\text { extraterrestrial }^{\text {(a) }}\end{array}$ & $\begin{array}{l}\text { air, none, } \\
\text { small } \\
\text { water } \\
\text { content }\end{array}$ & $10^{-3}-10^{1}$ & $\begin{array}{l}\sim 2000- \\
3000\end{array}$ & $\sim 0.4-0.7$ & $\begin{array}{l}10^{0}-10^{10} \\
10^{9}-10^{13(a)}\end{array}$ & $10^{-1}-10^{2}$ & $10^{-1}-10^{2}$ & $\begin{array}{l}10^{0}-10^{1} \\
10^{1}-10^{2}\end{array}$ \\
\hline $\begin{array}{l}\text { Submarine } \\
\text { landslides }\end{array}$ & subaqueous & water & - & - & - & $10^{0}-10^{13}$ & . & $10^{-1}-10^{2}$ & $10^{1}-10^{2}$ \\
\hline $\begin{array}{l}\text { Turbidity } \\
\text { currents }\end{array}$ & subaqueous & water & $10^{-4}-10^{-1}$ & $\begin{array}{l}\sim 1500- \\
2500\end{array}$ & $\begin{array}{l}\sim 0.001- \\
0.1\end{array}$ & $10^{6}-10^{10}$ & $10^{0}-10^{1}$ & $10^{1}-10^{2}$ & $10^{1}-10^{3}$ \\
\hline $\begin{array}{l}\text { Snow } \\
\text { avalanches } \\
\text { (dense }^{(b)} \text {, } \\
\text { powder }^{\left(c^{2}\right)}\end{array}$ & subaerial & air (water) & $10^{-4}-10^{-1}$ & $\begin{array}{l}\sim 100- \\
1000\end{array}$ & $\begin{array}{l}\sim 0.1- \\
0.4^{\text {(b) }} \\
\sim 0.001- \\
0.01^{\text {(c) }}\end{array}$ & $10^{4}-10^{6}$ & $10^{1}-10^{2}$ & $10^{0}-10^{1}$ & $10^{-1}-10^{0}$ \\
\hline $\begin{array}{l}\text { Pyroclastic } \\
\text { density } \\
\text { currents } \\
\text { (dense }^{(\mathrm{d})} \text {, } \\
\text { dilute }^{(\mathrm{e})} \text { ) }\end{array}$ & $\begin{array}{l}\text { subaerial, } \\
\text { subaqueous, } \\
\text { extraterrestrial }\end{array}$ & $\begin{array}{l}\text { volcanic } \\
\text { gases, air }\end{array}$ & $10^{-6}-10^{0}$ & $\begin{array}{l}\sim 500- \\
3000\end{array}$ & $\begin{array}{l}\sim 0.1- \\
0.5^{(\mathrm{d})} \\
\sim 0.001- \\
0.01^{(\mathrm{e})}\end{array}$ & $10^{4}-10^{8}$ & $\begin{array}{l}10^{0}-10^{1(\mathrm{~d})} \\
10^{1}-10^{2(\mathrm{e})}\end{array}$ & $10^{0}-10^{2}$ & $10^{0}-10^{2}$ \\
\hline $\begin{array}{l}\text { Debris } \\
\text { flows, } \\
\text { lahars }\end{array}$ & $\begin{array}{l}\text { subaerial, } \\
\text { extraterrestrial }\end{array}$ & water & $10^{-4}-10^{0}$ & $\begin{array}{l}\sim 2000- \\
3000\end{array}$ & $\sim 0.2-0.8$ & $10^{4}-10^{9}$ & $10^{0}-10^{1}$ & $10^{0}-10^{1}$ & $10^{0}-10^{2}$ \\
\hline
\end{tabular}

${ }^{\mathrm{a}-\mathrm{e}}$ See corresponding values of parameters.

Table 1 presents the main types of geophysical flows and gives ranges of values of the most relevant parameters (see some examples in Figures 1 and 2). The broad terminology associated with these flows reflects their great variety in terms of nature, triggering mechanisms, environments, temporal and spatial scales, and associated mechanical behavior (e. g. Coussot and Meunier (1996), Hungr et al. (2001, 2013)). We focus in this paper on the propagation phase while the study of the initiation phase is a crucial problem at the heart of hazard assessment studies that would require a paper in itself. The terminology considered here is that commonly used in literature based on the nature of the material involved, on the modes of flow generation and on dynamical aspects, but the meaning of the terms may differ between different geophysical communities. Some basic information is given here as a guide for the reader because the different types of flows will be referred to hereafter. Subaerial landslides, rock or debris avalanches are generated by the gravitational destabilization of cliffs, mountain slopes, and volcanic edifices. Rockfalls, rock or debris avalanches are dense granular flows for which the interstitial air or water, if present, is assumed to have a negligible influence on the dynamics (Hsü (1975), Hayashi and Self (1992)), while the term landslide may be used for dense granular flows partially filled or not with water. On Earth, these flows can be very small (rockfalls) but may also have volumes up to $\sim 10^{9} \mathrm{~m}^{3}$ and may contain ice from glaciers at high elevation and/or latitudes (Huggel et al. (2008), Favreau et al. (2010), Moretti et al. (2012)). Extraterrestrial events such as those on the planet Mars may also be very small (Mangold et al. (2010), Johnsson et al. (2014)) or reach huge volumes of up to $\sim 10^{13} \mathrm{~m}^{3}$ (McEwen (1989), Lucas et al. (2011, 2014)). In submarine 
environments, landslides initiate on continental margins or on oceanic ridges, are assumed to be more or less coherent solid masses, and may be more voluminous than their subaerial equivalents (Hampton et al. (1996), Masson et al. (2006)). While submarine landslide activity is rarely observed, recent high resolution surveys of underwater depth of ocean floors reveals small to large landslide deposits that shape the morphology of medio-oceanic ridges (Cannat et al. (2013)). In contrast, turbidity currents are generally smaller flows with low particle volume fraction (Normack et al. (1993), Meiburg and Kneller (2010), Cantero et al. (2012)). Snow avalanches can be dry or wet and the distinction is made between dense slab avalanches, of moderate thickness and high particle concentration, and powder snow avalanches of larger thickness and much lower particle concentration (Hopfinger (1983), Ancey (2001), Schweizer et al. (2003)). Volcanoes generate hot mixtures of volcanic gas and particles, called pyroclastic density currents, through lateral explosion of a magma body or the gravitational collapse of a lava dome or of an eruptive column (Druitt (1998), Branney and Kokelaar (2002), Roche et al. (2013a), Dufek (2016)). Pyroclastic flows and surges represent the dense and dilute end-members of mechanisms, respectively, and often coexist as a dense underflow is commonly overridden by a dilute turbulent ash cloud. Debris flows are dense mixtures of water and solid particles generated in various subaerial environments on Earth and on other planets (Iverson (1997), Iverson et al. (1997), Zanuttigh and Lamberti (2007), Mangold et al. (2010)) and they can derive from landslides (Scott et al. 2001). Lahars are debris flows resulting from the mixing of pyroclastic material with water during subglacial volcanic eruptions or remobilization of pyroclastic deposits by rains (Doyle et al. (2011), Lube et al. (2012)).

Geophysical flows can incorporate their ambient (i.e. surrounding) fluid and/or the substrate on which they propagate. Entrainment of the ambient fluid into a turbulent flow, in both aerial and subaqueous settings, decreases the particle concentration (along with sedimentation) and increases the flow thickness (Hallworth et al. (1993)). It occurs preferentially when the bulk density of the flow is close to that of the ambient fluid, which favors the growth of instabilities at the flow-ambient interface. Hence, fluid entrainment may be particularly efficient in turbidity currents, powder snow avalanches, and dilute pyroclastic density currents (Sparks et al. (1993)). In the latter case, heating and thermal expansion of the entrained air can render the flow buoyant, which leads to spectacular ascending plumes (Woods and Wohletz (1991)).

Geophysical flows such as turbidity currents, snow avalanches, and pyroclastic density currents often have a downward increasing concentration in particles as they consist in a relatively dense basal layer and a more dilute upper layer (Valentine (1987), Nishimura and Ito (1997), Gladstone and Sparks (2002)). The nature of the gradient (gradual or sharp) and the degree of interaction between the two layers are poorly understood. The particles in the currents are often of different natures (rock, pumice, sand, ice, etc.) and shapes. This is likely to affect their coefficients of friction and elastic restitution, for instance, which may in turn affect the flow behaviour and the morphology of the deposit (Goujon et al. (2007), Schneider et al. (2011)). They can also have different densities, typically in the range $1000-3000 \mathrm{~kg} \mathrm{~m}^{-3}$. An important characteristic of many geophysical flows is that their grain size range commonly spans several orders of magnitude due to the initial composition of the material released and to efficient fragmentation processes (Figure 2a-b). The smallest particles are often micrometric silts, clays, or ashes, whereas the largest blocks can be of metric to decametric size. Strong heterogeneities in particle size are observed in many deposits in the directions normal and parallel to the topography (Weidinger et al. (2014)). Furthermore, the particle volumetric concentration often changes drastically during emplacement. Segregation of the particles according to their size and/or density also modifies the arrangement of the granular network (e.g. Félix and Thomas (2004), Hill and Yohannes (2011), Johnson et al. (2012), Gray and Hutter (1997), Hill and Tan (2014), Hill and Fan (2016)). Note that segregation of large particles toward the top of the flow is commonly called "normal" by physicists (Brazil nut effect) and "reverse" by geophysicists or engineers, which may be a source of confusion in the literature. For instance, in gravity driven shear flows, Savage and Lun (1988) mention kinetic sieving and squeeze expulsion driving grains into an inversely graded configuration for large grains at the top and small grains at the bottom. Segregation, along with grain size range variations due to particle fragmentation and/or aggregation, can significantly change some flow properties such as hydraulic permeability, which plays a key role in the coupling of the granular and fluid phases. Indeed, pore pressure diffusion may be fundamental and its timescale depends essentially both on the flow hydraulic permeability and on the fluid viscosity and compressibility (e.g. 
Pierson (1986), Iverson (1997), Major and Iverson (1999), Iverson and Vallance (2001)). For instance, rapid pressure diffusion due to the permeability increase caused by segregation of coarse particles at the front and lateral margins of debris and pyroclastic flows is thought to cause frictional resistance higher than that in the main body (Major and Iverson (1999), Jessop et al. (2012)). The process of pore pressure diffusion when the particle concentration increases with time (i.e. deflating flows) and/or the interstitial fluid is highly compressible (i.e. gas-particle flows) is yet to be well characterized (e.g. Montserrat et al. (2012)). In these cases, some fundamental insights can be gained from laboratory experiments (e.g. Pailha et al. (2008), Pailha and Pouliquen (2009), Roche (2012), Kaitna et al. (2014), Valverde and Soria-Hoyo (2015)).

Interaction of the flows with their underlying substrate is evidenced by impact marks and scoured surfaces (Sparks et al. (1997), Calder et al. (2000), Pittari and Cas (2004), Le Friant et al. (2004), Conway et al. (2010), Eggenhuisen et al. (2011)). Geophysical flows can also incorporate underlying substrates made of unconsolidated granular materials that often result from earlier events, which increases the mass of the flowing material (Gauer and Issler (2004), Hungr and Evans (2004), Mangeney et al. (2007b, 2010), Berger et al. (2011), Doyle et al. (2011), Mangeney (2011), Iverson (2012), McCoy et al. (2012), Roche et al. (2013a), Farin et al. (2014), Edwards and Gray (2015)). For instance, the mass of snow avalanches on steep slopes can increase by a factor of four through entrainment (Sovilla et al. (2006)). Features typical of basal frictional heating and melting may be evidence of reduced friction due to lubrification of the contacts between the particles or to increase of pore fluid pressure (Legros et al. (2000), Goren and Aharonov (2007), De Blasio and Elverhøi (2008), Goren et al. (2010), Weidinger et al. (2014), Lucas et al. (2014)).

The high variety of geophysical flows renders the development of a unified understanding and theoretical description of these phenomena very difficult. Besides their diversity, natural flows are fundamentally non-permanent, transient phenomena whose propagation is characterized by three steps (i.e., initiation, propagation, and deposition) that commonly involve different physical processes. Initiation spans from nearly instantaneous release of material to continuous supply that can last up to several minutes. Propagation can be characterized by complex flow dynamics, as shown for instance by long-lived debris flows for which surface waves may develop so that several pulses may arise from a single event (Zanuttigh and Lamberti (2007)), or by complex interaction with the underlying topography (Favreau et al. (2010), Moretti et al. (2015)) and an erodible substrate (Moretti et al. (2012)). Deposition typically does not occur everywhere at the same time due to spatio-temporal variations of the flow properties and/or topographical effects as parts of the material come to halt while others are still moving. This is the case for example in self-channelling flows where static lateral levees develop bordering a mobile flow in a channel, partly due to segregation generating coarsegrained levees (Pouliquen et al. (1997), Félix and Thomas (2004), Mangeney et al. (2007a), Jessop et al. (2012), Johnson et al. (2012), Woodhouse et al. (2012), Kokelaar et al. (2014)).

The propagation and energy dissipation of geophysical granular flows are controlled simultaneously by (i) interactions between the particles (friction and collisions) and with the underlying bed, (ii) interactions between the particles and the interstitial fluid filling the interstices between them, (iii) viscous shear of the interstitial fluid (Iverson (1997)), and (iv) shear with the ambient fluid. The interstitial fluid can be different from the ambient fluid. It is either a liquid or a gas (possibly containing fine particles), which is commonly water or air, so that differences in fluid density and viscosity promote various intensities of stresses. The interstitial fluid phase is particularly critical in the dense regime as interstitial pore fluid pressure may arise and damp particles interactions, especially when the fluid is highly viscous and/or compressible. Note that water possibly incorporated during propagation may transform into vapor due to frictional heating or in case of a hot flow.

The measurement and theoretical description of these flows and of the physical processes involved in a natural environment are still open and extremely challenging problems for earth scientists, giving rise to equally challenging physical issues. Table 2 highlights key questions related to these phenomena in the domain of physics and geophysics. This somehow arbitrary separation between physical and geophysical aspects reflects the extreme complexity and variety of the natural flows as well as the difficulty to relate field observation to simple physical experiments and theoretical description. In other words the relevance of using small-scale experiments to model natural flows is questionable. Most of the physical studies on granular flows consider significant simplifications regarding the material properties and the boundary conditions. They commonly involve unidirectional 
flows of spherical particles on inclined planes of a length of a few meters (see section 3). The grain size range is often slightly polydisperse and the flow underlying boundary is either flat or covered with glued beads while the influence of lateral walls is often ignored. Even in these very simple systems, a description of the flow in term of rheology, energy dissipation mechanisms, jamming, and flow regime transitions remains essentially incomplete. Field observations by geologists and geophysicists or simple granular flow experiments or models by physicists both provide an extremely simplified view of the natural physical processes at work. One of the major challenges is to make the link between these complementary approaches by taking advantage of the wealth of field data on natural events and of the recent development in the physics of granular flows. Beyond the establishment of constitutive relations that can be used to simulate natural flows, the experimental, theoretical and numerical investigations on simple granular systems may highlight some key processes that guide interpretation of field data.

Table 2: Main questions related to granular geophysical flows, schematically separated into geophysical and physical issues.

\section{Geophysics}

How to detect geophysical flows and to assess their related hazards (i.e., flow runout distance, area of deposit, impact pressure on structures, etc.) and indirect impact (tsunamis, etc.)?

What is the contribution of gravitational flows in erosion processes and relief evolution at the surface of the Earth and other planets?

How are gravitational flows related to external forcing (climatic, volcanic, seismic activity)? Could they provide indicators or precursors of these forcing processes?

What physical processes may be at the origin of the high mobility of large natural landslides?

How to quantify and model erosion/deposition processes, solid/fluid interaction, polydispersity and fragmentation at the natural scale?

How to retrieve the mechanisms of propagation and the characteristics of the flows (mass, presence of water, erosion processes, initiation processes, degree of particle-particle and fluid-particle interactions, etc.) from their deposit and/or from the generated seismic or geophysical signal on Earth and on other planets ?

\section{Physics}

What is the role played by the interstitial fluid according to the nature of the flow, and conversely, both being coupled? How to describe the grains/fluid coupling, taking into account in particular dilatation/compression effects?

Can we obtain constitutive relations giving a complete description of the granular flows and of their transitions (jammed, dense, dilute)?

How can be captured the boundary conditions and how do they affect the flow? This includes fixed interfaces that are generally associated to an effective friction, and mobile interfaces related to erosion/deposition processes.

How to quantify and describe theoretically the evolution of granular size distribution in space and time (segregation, fragmentation processes) and its coupling with the flow?

\section{Physics and Geophysics}

How to measure granular and fluid stresses, particle volume fraction, etc. in both experimental and natural flows? 

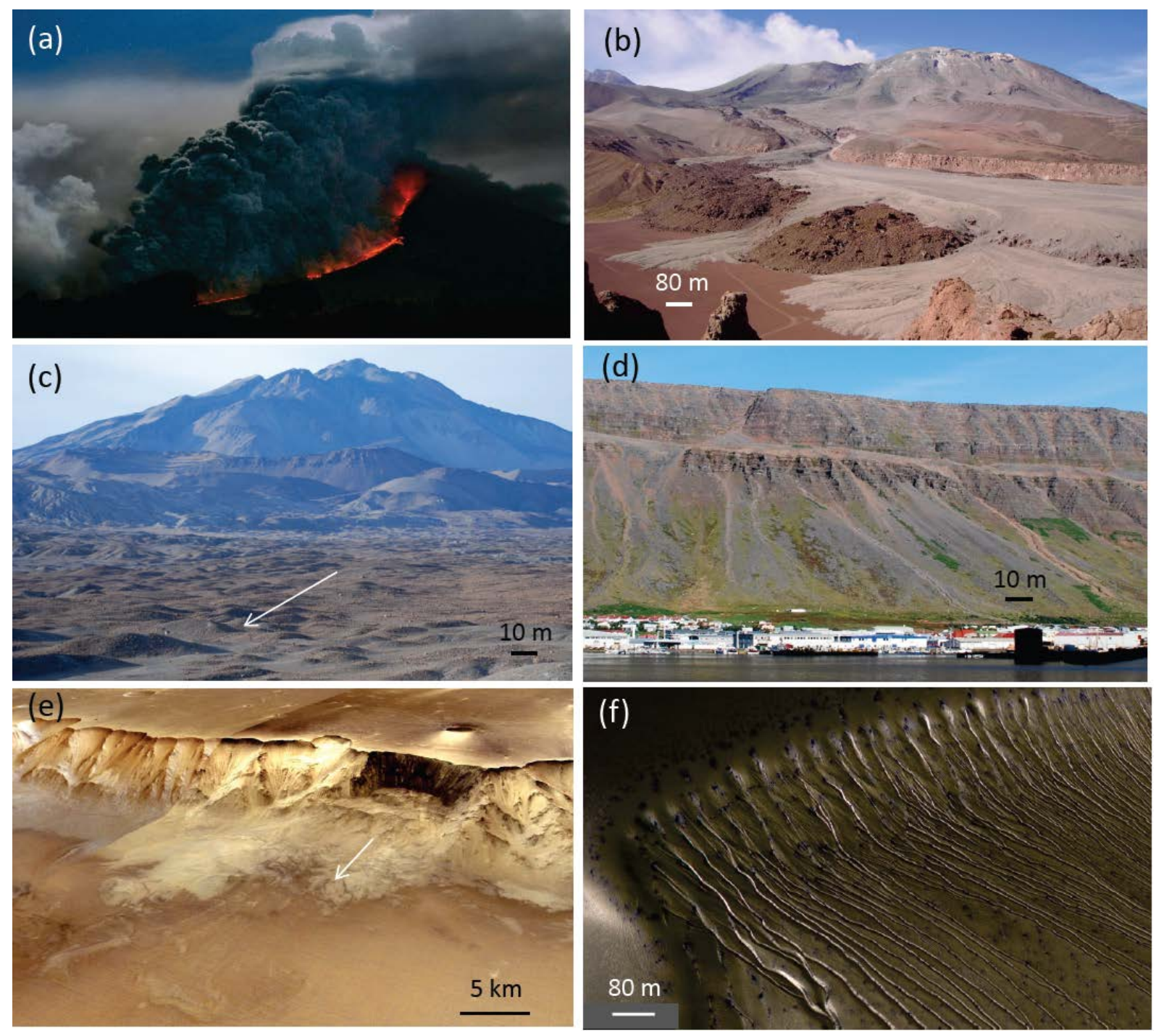

Figure 1. Example of natural granular gravitational flows. (a) Pyroclastic flows at dawn in January 2014 on Sinabung volcano, Indonesia. Note the basal hot flow and the upper ash cloud; picture by O. Grunewald. (b) Deposits of the 1993 pyroclastic flows on Lascar volcano, Chile (Jessop et al. (2012)). (c) Hummocky deposits of the $26 \mathrm{~km}^{3}$ Socompa debris avalanche. (d) Erosive debris flows threatening the downslope village of Isafjordur in Iceland ; pictures by A. Mangeney. (e) Ganges Chasma landslide in Valles Marineris on the planet Mars ( $7^{\circ} 15 \mathrm{~N} ; 50^{\circ} 28 \mathrm{~W}$ ) - Image HRSC/DLR/ESA (Lucas et al. (2014)). (f) Gullies on the mega-dune of Russell crater on the planet Mars $\left(5^{\circ} 15 \mathrm{~N} ; 1^{\circ} 52 \mathrm{E}\right)$ - Image HiRISE/NASA/JPL-Caltech/Univ. of Arizona (Lucas (2010)). 

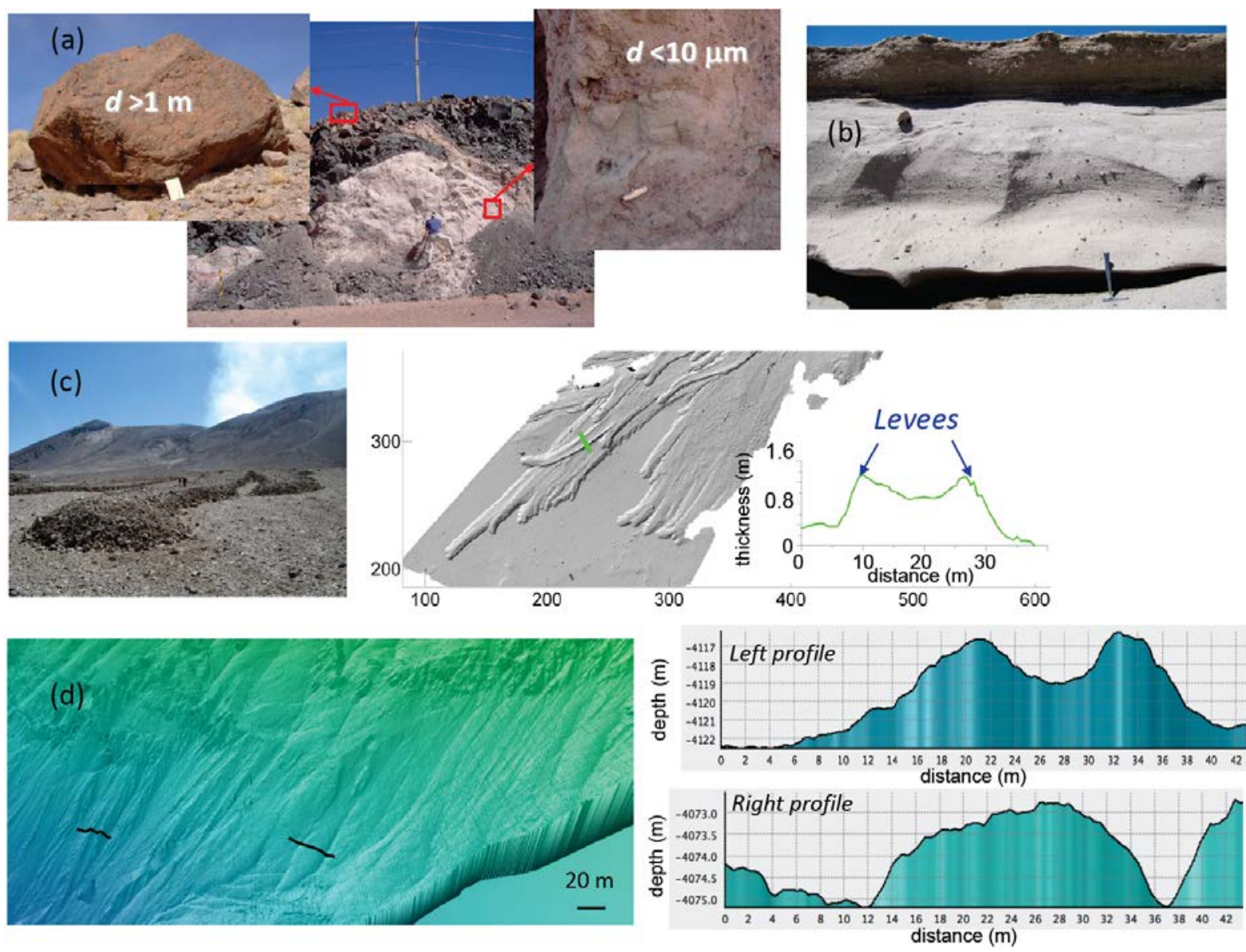

Figure 2. (a) Deposit of the Socompa debris avalanche, Chile, showing the polydispersity of the deposit with boulders of diameter $d$ larger than $1 \mathrm{~m}$ to fine particles of diameter smaller than $10 \mu \mathrm{m}$; a zoom within the matrix made of small particles shows a block very well preserved while the block itself and the surrounding matrix are completely pulverized; picture: A. Mangeney. (b) Pyroclastic flow deposit consisting of a matrix of fine ash (size $<500 \mu \mathrm{m})$ with blocks (>10 cm), Tutupaca volcano, Peru; picture: O. Roche. (c) Front of the 1993 Lascar pyroclastic flow deposits. Morphometry (right) obtained by scanner laser measurements showing the lateral levees surrounding a central channel of lower thickness (Jessop et al. (2012)). (d) Submarine flow deposits on the Mid-oceanic ridge in Krasnov with levee-channel and rounded deposits (Cannat et al. (2013)).

\section{Physical Concepts, Theoretical and Numerical modeling}

\subsection{Forces acting on particles}

2.1.1 Particle/particle forces. In dry granular flows, particles interact via contact forces including collisions and enduring contacts. A collision involving two macroscopic grains is inelastic (in contrast to collision of molecules) and thus dissipates energy. The dissipation is commonly characterized by a coefficient of restitution, $e$, which is defined as the ratio of the post-collision relative velocity to the pre-collision relative velocity (Louge and Keast (2001)). In dilute granular flows, energy dissipation occurs primarily via dissipative binary collisions. In contrast, in dense granular flows, particles remain in contact within a finite time and dissipate energy via enduring contacts which involve primarily solid friction. Dry solid friction is characterized by a friction coefficient, $\mu$, defined as the ratio of the shear force to the normal force required to initiate sliding, which is material dependent.

2.1.2 Fluid/particle forces. In the presence of an interstitial fluid, particles are subjected to additional forces. First, a particle undergoes a fluid resistance force which is opposite to its relative motion. This drag force, $F_{\mathrm{d}}$, can be expressed formally as:

$$
F_{\mathrm{d}}=C\left(u_{\mathrm{f}}-u_{\mathrm{p}}\right)
$$


where $u_{\mathrm{p}}$ and $u_{\mathrm{f}}$ are the velocities of the particle and fluid, respectively. The factor $C$ depends on various parameters such as the particle Reynolds number, the local solid volume fraction $\phi$, etc. For dilute flows of smooth spherical particles, at small particle Reynolds number, the fluid drag reduces to the Stokes force ( $C=3 \pi d \eta_{\mathrm{f}}$, where $\eta_{\mathrm{f}}$ is the fluid dynamic viscosity and $d$ the grain diameter), while at high Reynolds particle number, the drag is independent of the fluid viscosity and scales quadratically with the relative particle velocity ( $C \approx 0.5(\pi / 8) d^{2} \rho_{\mathrm{f}}\left(u_{\mathrm{f}}-u_{\mathrm{p}}\right)$, where $\rho_{\mathrm{f}}$ is the fluid mass density). Note that (2.1) holds in principle only for steady particle motion. For unsteady motion, additional forces should be considered, such as the virtual mass effect and the Basset force (Johnson (1998)).

Second, when two particles come close to each other, they may undergo a repulsive force known as lubrication force. This force arises from hydrodynamic pressure in the interstitial fluid being squeezed out from the space between two particles and is expressed as:

$$
F_{\mathrm{L}} \approx(3 \pi / 2) \eta_{\mathrm{f}} \delta u_{\mathrm{p}} / h
$$

where $h$ is the gap between the particles and $\delta u_{\mathrm{p}}$ their relative approaching velocity. As a consequence, the interparticle contact forces may be drastically altered in the presence of an interstitial fluid. Gondret et al. (2002) showed experimentally that the dissipation in a binary collision of particles immersed in a fluid is increased in comparison with the dry case and can be expressed in terms of an effective restitution coefficient $e_{\text {eff }}$ which follows a master curve depending solely of the Stokes number $S t=\rho_{\mathrm{p}} \delta u_{\mathrm{p}} d / \eta_{\mathrm{f}}$ where $\rho_{\mathrm{p}}$ is the particle mass density. At small Stokes number (i.e. $S t<10$ ) the ratio $e_{\text {eff }} / e_{\text {dry }}$ drops to zero, it approaches 1 at high Stokes number $\left(S t>10^{4}\right)$ and, on a relatively large interval, $e_{\text {eff }}$ can be approximated by (Yang and Hunt (2006)):

$$
e_{\mathrm{eff}} \approx e_{\mathrm{dry}}-62\left(1+e_{\mathrm{dry}}\right) / S t
$$

If the interstitial fluid is the air, lubrication force can be safely neglected for millimeter particle size, whereas in a liquid, the latter may play a significant role.

Third, particles may be sensitive to the turbulent fluctuations of the fluid flow. This is the case for fine particles in a turbulent fluid flow. The associated transport is called "turbulent suspension". The dimensionless number for determining whether turbulent suspension is effective or not is the Rouse number: Rouse $=w_{\mathrm{p}} / \mathrm{Ku}_{*}$. It is a ratio between the particle fall velocity $w_{\mathrm{p}}$ and the vertical fluctuating grain velocity as a product of the von Karman constant $\kappa$ and the shear velocity $u *$. Rouse number smaller than unity indicates that turbulent suspension is effective.

\subsection{Flow classification and dimensionless numbers}

Among granular flows and particle liquid mixtures, three major categories of flows can be identified according to their solid fraction (the typical values given here correspond to spherical monosized particles): very dilute $(\phi<0.01)$, dilute $(0.1<\phi<0.5)$ and dense flows $(0.5<\phi<0.59)$. For very dilute flows, particle/particle interactions play a minor role. These flows include dilute turbidity currents, powder snow avalanches and pyroclastic currents. The motion of particles is driven by the turbulent liquid flow and gravity forces. On the contrary, for dilute and dense flows, particle/particle interactions play an important role. These flows include aerial rock and debris avalanches, landslides, submarine avalanches, debris flows and dense pyroclastic currents. Dense flows can be divided into three subcategories depending on the nature of the particle/particle interactions: (i) particle inertiadominated regime, (ii) viscous resistance-dominated regime and (iii) fluid inertial resistancedominated regime. This classification is based on the analysis of the time scale of the particle displacement (see Courrech du Pont et al. (2003a) and Cassar et al. (2005) for further details).

For these different regimes, we can introduce a dimensionless ratio $I$ defined as the ratio of the characteristic particle time scale to the characteristic time equal to the inverse of the flow shear rate $\dot{\gamma}$ (for sake of simplicity we consider here a simple shear flow): 
- Particle inertia-dominated regime (i): $I=I_{\mathrm{pi}}=\sqrt{\rho_{\mathrm{p}} \dot{\gamma}^{2} d^{2} / P_{\mathrm{p}}}$

- Viscous resistance-dominated regime (ii): $I=I_{\mathrm{v}}=\frac{\eta_{\mathrm{f}} \dot{\gamma}}{P_{\mathrm{p}}}$

- Fluid inertia-dominated regime (iii): $I=I_{\mathrm{fi}}=\sqrt{\rho_{\mathrm{f}} \dot{\gamma}^{2} d^{2} / P_{\mathrm{p}}}$.

$P_{\mathrm{p}}$ is the confining pressure, $\rho_{\mathrm{p}}$ and $\rho_{\mathrm{f}}$ are the particle and fluid density respectively. Note that we drop the numerical factor in the definition of I to make it consistent with the usual definition of inertial number (see Eq. (2.10)). The inertial regime (or Bagnoldian regime) has raised a great interest in the granular physics community and various theoretical approaches have been developed to model this flow regime. Two emblematic approaches are the granular kinetic theory for collisional granular flows and the so called " $\mu(I)$ ” rheology for dense granular flows.

Alternative dimensionless numbers have been introduced in the literature (Iverson (1997)) on the basis of the comparison of particle and fluid stresses. One can mention the Savage and Bagnold number defined respectively as the ratio of the inertial grain shear stress to the weight of flowing layer per unit surface

$$
N_{\text {Sav }}=\frac{\rho_{\mathrm{p}} \dot{\gamma}^{2} d^{2}}{\left(\rho_{\mathrm{p}}-\rho_{\mathrm{f}}\right) g H}
$$

and the ratio of inertial grain shear stress to viscous shear stress,

$$
N_{\text {Bag }}=\frac{\phi \rho_{\mathrm{p}} \dot{\gamma}^{2} d^{2}}{(1-\phi) \eta_{\mathrm{f}} \dot{\gamma}}
$$

where $H$ is the thickness of granular layer. The Savage number $N_{\text {Sav }}$ is nothing but the square of the inertial number $I_{\mathrm{pi}}$ in which the confining pressure is driven by the hydrostatic force $\left(P_{\mathrm{p}} \sim\left(\rho_{\mathrm{p}}-\rho_{\mathrm{f}}\right) g H\right.$ ). The Bagnold number is a simple combination of $I_{\mathrm{pi}}$ and $I_{\mathrm{v}}: N_{\mathrm{Bag}} \sim I_{\mathrm{pi}}{ }^{2} / I_{\mathrm{v}}$. High Bagnold number thus refers to particle inertia-dominated regime.

\subsection{Dry granular flows}

We focus here on the inertial regime, where the stress due to the grains is far larger than that exerted by the fluid, so that one may ignore the effects of the ambient fluid and consider inter-particle interactions only.

2.3.1 Collisional Granular flows: kinetic theory. In rapid granular flows, the grains interact mainly through binary collisions in a way that is reminiscent of molecules interacting in a gas. There are nevertheless significant differences between a "granular gas" and a molecular gas. The disparity of sizes between grains and molecules is not the direct cause of these differences. The fact that grain collisions are not elastic, and thus dissipative, has major implications concerning the behaviour of grain assemblies: granular gases are always in non-equilibrium states.

It is common to define the granular temperature in a slightly different way than for molecular gases: $T$ is defined as the average (over volumes and time ranges) of the square of the grain velocity fluctuations. This statistical definition does not need to refer to a state of equilibrium. One of the main applications of the concept of granular temperature is the construction of kinetic theories. The term "kinetic theory" is often used in different contexts. The more general one is a mesoscopic approach where the state of the system is described using the distribution function specifying the distribution of particle positions and velocities. In most cases, it cannot be solved exactly. The other one, called Chapman-Enskog expansion, is an approximation where the two particle distribution function is expressed as the product of the single particle velocity distribution functions, and the pair distribution at contact. This approach neglects the correlations. The hydrodynamic equations of motion resulting from the application of these theories resemble the compressible Navier-Stokes equations: 


$$
\begin{aligned}
& \partial\left(\rho_{\mathrm{p}} \phi\right) / \partial t+\nabla \cdot\left(\rho_{p} \phi \mathbf{u}\right)=0 \\
& \rho_{\mathrm{p}} \phi(\partial \mathbf{u} / \partial t+\mathbf{u} \cdot \nabla \mathbf{u})=\nabla \cdot \boldsymbol{\sigma}+\mathbf{f}
\end{aligned}
$$

Here, $\phi$ is the volume fraction, $\mathbf{u}$ the mean velocity, $\boldsymbol{\sigma}$ the stress tensor and $\mathbf{f}$ the body force density. The equation for the energy density, $e$, contains a sink term $\Gamma_{d}$ that represents the loss of energy due to the inelasticity of the collisions (q is the energy flux vector):

$$
\rho_{p} \phi(\partial e / \partial t+\mathbf{u} \cdot \nabla e)=-\nabla \cdot \mathbf{q}+\boldsymbol{\sigma}: \nabla \mathbf{u}-\Gamma_{d}
$$

This term is responsible for the existence of many phenomena that characterize granular gases. The hydrodynamic description is completed by constitutive relations expressing $\sigma$, $\mathbf{q}$ and $\Gamma_{d}$ in terms of the hydrodynamic fields. Kinetic theory provides local constitutive relations through the application of the Chapman-Enskog expansion. The constitutive relation for $\tau$ is expressed as in a Newtonian fluid:

$$
\boldsymbol{\sigma}=[-p+(\lambda-2 \eta / 3) \nabla \cdot \mathbf{u}] \mathbf{1}+\eta\left[\left(\nabla \mathbf{u}+(\nabla \mathbf{u})^{T}\right)\right]
$$

The energy flux vector $\mathbf{q}=-\kappa \nabla \cdot T$, the pressure $p=\rho_{\mathrm{p}} \phi\left(1+4 \phi g_{12}\right) T$, the viscosities $\lambda$ and $\eta$, the conductivity $\kappa$ and the sink term $\Gamma_{\mathrm{d}}$ are given in terms of the volume fraction, the granular temperature $T=2 e / 3$ and the pair distribution at contact $\mathrm{g}_{12}(\phi)$ (Jenkins and Savage (1983), see also Delannay et al. (2007) and references herein).

The solutions of these equations have had some success in describing relatively dilute flows or moderately dense flows in the absence of gravity (Azanza et al. (1999), Forterre and Pouliquen (2001), Xu et al. (2003)).

In the dense limit, this approximation generally fails. An improvement of the models to incorporate the effect of correlations is necessary to describe the dense flow regime. The challenges for applying such ab initio theories for dense granular flows are discussed in Delannay et al. (2007) and Kumaran (2015).

2.3.2 Dense granular flows. In the dense flow regime, collisions still exist, but they can involve more than two bodies and become so frequent that very strong energy dissipation occurs (inelastic collapse). Contacts between particles tend to be enduring and long-lasting; the contact network can percolate through particles. The enduring frictional contacts induce correlations between the velocities and/or locations of the particles.

There have been several attempts to extend the simple kinetic theory to include higher volume fractions, greater dissipation in collisions, and multiple or enduring contacts. The existing constitutive relations of the kinetic theory need to be modified by the incorporation of the correlations associated with the enduring and/or repeated contacts. However, the appropriate form of this modification has yet to be determined. Up to now no constitutive equations are available in the dense regime.

Several studies (Da Cruz et al. (2005), GDR MiDi (2004)) have pointed out that simple dimensional arguments could provide an interesting framework for constitutive laws for dense granular flows. These may be phrased as empirical constitutive relations for the shear stress and normal stress in steady homogeneous shearing. An assembly of rigid frictional spheres of diameter $d$ and mass density $\rho_{\mathrm{p}}$, confined under a normal stress $P$ in between two bumpy planes, is sheared at a given shear rate $\dot{\gamma}$ by applying a shear stress $\tau$. If one neglects the finite size of the sample, the nondimensional inertial number $I$ defined above (sec. 2.2) is the only dimensionless parameter of the problem. As a consequence, the shear stress has to be proportional to $P$ times a function of $I$. The ratio of tangential to normal stresses is the effective coefficient of friction $\mu$ :

$$
\tau / P=\mu(I) \text { with } I=\dot{\gamma} d / \sqrt{P / \rho_{p}}
$$


The form of the function $\mu(I)$ can be obtained from the numerical simulations of plane shear (Da Cruz et al. (2005)) or indirectly by experimental measurements for flow down inclined planes (cf. section 3.2). The function $\mu(I)$ goes from a minimum value $\mu_{\mathrm{s}}$ for very low $I$ (quasi-static regime) up to an asymptotical finite value $\mu_{2}$ when $I$ increases. It can be expressed by the following expression (Jop et al. (2005)):

$$
\mu(I)=\mu_{S}+\left(\mu_{2}-\mu_{S}\right) /\left(1+I_{0} / I\right)
$$

where $I_{0}$ is a constant. The values of coefficients are material-dependent, for instance typical values for glass bead are $\mu_{\mathrm{s}}=\tan \left(21^{\circ}\right), \mu_{2}=\tan \left(33^{\circ}\right)$ and $I_{0}=0.28$ (GDR Midi (2004), Jop et al. (2005)).

Another relation, also derived from dimensional analysis, gives the variation of the volume fraction; $\phi$ is, in general, an affine decreasing function of $I$ (Da Cruz et al. (2005)). This relation completes the constitutive laws:

$$
\phi=\phi_{\max }-\left(\phi_{\max }-\phi_{\min }\right) I
$$

Typical values are $\phi_{\max }=0.6$ and $\phi_{\min }=0.5$ (Pouliquen et al. (2005)). The latter equation is only for $I$ lower that 1, i.e. for volume fraction larger than $\phi_{\text {min }}$.

A generalization of this approach to tensorial constitutive relations for incompressible 3D flows has been proposed (Jop et al. (2006)):

$$
\boldsymbol{\sigma}=-P \mathbf{I}+\boldsymbol{\tau} \text {, with } \boldsymbol{\tau}=\dot{\boldsymbol{\gamma}} \text {, and } \eta=\frac{\mu(I) P}{\|\dot{\gamma}\|}, I=\frac{\|\dot{\gamma}\| d}{\sqrt{P / \rho_{p}}}
$$

$\mathrm{P}$ represents an isotropic pressure, by definition it is the negative one-third the trace of the stress tensor, and $\tau$ is the deviatoric component. The effective viscosity $\eta$ is a function of the second invariant $\|\dot{\gamma}\|$ of the strain rate tensor $\dot{\gamma}$. Its definition is related to the friction coefficient law $\mu(I)$ (2.11).

This relation can be extended for the compressible case by replacing the strain rate tensor by its deviatoric component in (2.13) (Cortet et al. (2009), Börzsönyi et al. (2009)). It implies, in addition, a univocal dependence of the volume fraction on the inertial number.

Considering (2.13), in the limit of a shear rate going to zero, one can see that the material flows only if the following Drucker-Prager-like yield criterion is satisfied: $\|\tau\|>\mu_{\mathrm{s}} P$. Below the threshold, the medium behaves locally as a rigid body. Note that the constitutive relation (2.13) can be separated into two terms: a Drucker-Prager yield stress term involving the constant friction coefficient $\mu_{\mathrm{s}}$, and a viscous term with a viscosity depending on both the pressure and the norm of the strain rate tensor (Ionescu et al. (2015)). The specificity compared to classical Bingham or Herschel-Bulkley fluids is that the yield stress depends on the local pressure and the effective viscosity depends both on the shear rate and on the local pressure.

The $\mu(I)$ rheology had some success in reproducing quantitatively experimental observations in relatively simple situations such as granular column collapses (cf. section 3.4.2). Nevertheless, (2.13) contains very strong assertions: stress and strain rate tensors have to be aligned, and the second invariants should satisfy the relation:

$$
\frac{\|\boldsymbol{\tau}\|}{P}=\mu(I)
$$

where the function $\mu(I)$ follows (2.11). The tensorial relation (2.13) has been tested by numerical simulations using DEM (see section 2.7) in different situations (Cortet et al 2009, Börzsönyi et al. (2009)). It turns out that the alignment between stress and strain rate tensors fails. Interestingly, the relation (2.14) works better. But it does not seem to give a friction coefficient law similar to (2.11). A logarithmic decay is observed (Cortet et al. (2009)) for vanishing values of $I$ (leading to very low 
values of the friction coefficient) and a non-monotonic variation of $\mu(I)$ is reported at larger values of $I$ (with high value for the friction coefficient) (Börzsönyi et al. (2009)). A granular rheology that generalises the $\mu(I)$ model and incorporates first and second normal stress differences has been recently proposed (McElwaine et al. (2012)).

Another questionable point is that such a rheology does not use the notion of granular temperature which is at the base of the kinetic theory (Jenkins and Richman (1985)) even in the case of dense flows. The friction function is purely phenomenological without explicit links between the coefficients used and the grain properties. Moreover, the variation of the effective friction $\mu$ with the inertial number $I$ - which has been obtained empirically and not theoretically - has been recently questioned (Holyoake and McElwaine (2012)). Clearly, a link between the $\mu(I)$-rheology and the grain-scale physics is still missing. Note also that it has been recently shown (Barker et al. (2015)) that, in the incompressible limit, the $\mu(I)$ rheology is only mathematically well-posed for intermediate values of the inertial number. At high and low inertial numbers the equations are ill-posed, i.e. the growth rate of small perturbations increases infinitely fast in the high wavenumber limit. The practical significance of this is that two-dimensional time-dependent simulations in the ill-posed regime will exhibit short wavelength instabilities that will get progressively stronger as the resolution is enhanced, i.e. the results will be grid-dependent. This points out that there is some important missing physics (force chains, effect of grain stiffness) for both high and low inertial numbers where the problem is ill-posed.

The $\mu(I)$-rheology is a local rheology in its current form, it is thus not valid close to the jamming transition (Staron et al. (2010), Deboeuf et al. (2005)), or when non-local effects are not negligible (Nichol et al. (2010), Reddy et al. (2011)). As already mentioned, the enduring frictional contacts induce correlations between the velocities and locations of the particles. These spatial correlations become very important, both in the force network and velocity fluctuations, near the jamming transition. To incorporate these non-local effects in the constitutive relations is not an easy task. It is a generic question emerging in the study of other complex fluids near jamming: foams, emulsions, colloidal suspensions. Some of the attempts to develop non local theories are described in GDR MiDi (2004), Delannay et al. (2007), Henann and Karmin (2013) and Jop (2015). For example, Jenkins (2006, 2007) modified the kinetic theory by introducing a length associated with the size of particle clusters into the expression of the rate of collisional dissipation. This theory has been extended to very dissipative, frictional spheres (Jenkins and Berzi (2010)). The latter theory has the capacity to reproduce, at least qualitatively, experimental results of dense, unconfined, inclined granular flows over a rigid bumpy base and dense, confined flows over an erodible base (cf. section 3.2).

\subsection{Modeling particle- fluid mixtures}

2.4.1 Introduction. Debris flows, landslides or submarine avalanches are geophysical events characterized by the flow of a mixture of liquid and particles down a slope. The rheology of this mixture presents significant modeling challenge. We lack a full understanding of how these flows are initiated but there is a growing understanding of processes governing flows, once motion has been triggered.

2.4.2 Single-phase model. Single-phase models have been employed to describe fluid particle mixture. They are based on a unique rheological relation between the shear stress and the shear strain rate and consider the mixture as a continuum. They typically employ a non-newtonian rheology to incorporate the effect of grain interaction (Takahashi (1991), Coussot (1994), Chen and Ling (1998), Brufau et al. (2000)). The rheologies adopted range from rigid-viscous (yield, followed by a linear viscous stress, Bingham (1922)) to collisional (shear and normal stresses quadratic in the shear rate, Bagnold (1954)). These approaches become inappropriate when the fluid phase and the granular skeleton have a differential motion, inducing gradients of fluid pressure. The relative motion between the fluid and the particle phase can be created by various mechanisms: e. g. the development of hydrostatic pressure gradients or simply the compaction or dilation of the granular network. The last point is well illustrated when the flow is initiated or comes to rest as the solid volume fraction of the granular phase changes. Granular materials are indeed known to change volume when sheared: a dense packing dilates while a loose one compacts (Reynolds (1886)). When the granular material is saturated with a 
fluid, the change in volume fraction induces a pore pressure gradient (Iverson et al. (2000)), which in turn either alters the particle motion (dilation) or reduces interparticle friction (compaction).

2.4.3 Two-phase model. More elaborate models distinguish between the two phases and assume that they interact through drag and buoyancy. These models are referred to as "two-phase" or "two-fluid" models (Anderson and Jackson (1967), Drew (1983), Bedford and Drumheller (1983), Anderson et al. (1995), Jackson $(1997,2000)$ ) and are based on an averaging of mass and momentum balance laws for fluid and solid constituents.

Our purpose here is not to list all the two-phase models of the literature but rather to discuss about the different key assumptions made in these models. For this, we shall first focus on simple flow configurations corresponding to unidirectional, steady and fully developed flows before addressing issues concerning unsteady or inhomogeneous flows.

2.4.4 Steady and fully developed regimes. We consider a unidirectional, steady and fully developed flow down an incline, consisting of a mixture of granular material and interstitial fluid, each of constant specific mass density $\rho_{\mathrm{p}}$ and $\rho_{\mathrm{f}}$, respectively.

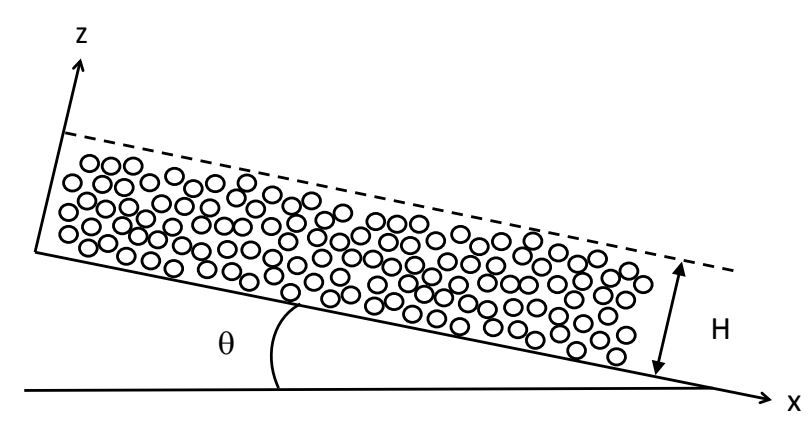

Figure 3. Sketch of a steady and fully developed flow down an incline of angle $\theta$

In this simple flow configuration, the momentum equations take the following forms,

$$
\begin{array}{r}
\frac{d \tau_{x z, \mathrm{p}}}{d z}+C \phi\left(u_{\mathrm{f}}-u_{\mathrm{p}}\right)+\rho_{\mathrm{p}} \phi g \sin \theta=0 \\
-\frac{d P_{\mathrm{p}}}{d z}-\phi \frac{d P_{\mathrm{f}}}{d z}+\rho_{\mathrm{p}} \phi g \cos \theta=0
\end{array}
$$

for the particle phase, and

$$
\begin{array}{r}
\frac{d \tau_{x z, \mathrm{f}}}{d z}-C \phi\left(u_{\mathrm{f}}-u_{\mathrm{p}}\right)+\rho_{\mathrm{f}}(1-\phi) g \sin \theta=0 \\
-(1-\phi) \frac{d P_{\mathrm{f}}}{d z}+\rho_{\mathrm{f}}(1-\phi) g \cos \theta=0
\end{array}
$$

for the fluid phase. $u_{\mathrm{p}}$ and $u_{\mathrm{f}}$ stand for the velocities of the solid and fluid constituents, respectively, $\tau_{x z, \mathrm{p}}$ and $\tau_{x z, \mathrm{f}}$ for the particle and fluid shear stress, $P_{\mathrm{p}}$ and $P_{\mathrm{f}}$ for the particle and fluid pressure, and $\phi$ for the solid volume fraction. The factor $C$ which quantifies drag, depends on various parameters (Reynolds number, solid fraction ...). The simplest form of the latter factor that incorporates viscous and form drag and concentration dependence reads as (Dallavalle (1943), Richardson and Zaki (1954)): 


$$
C=\frac{\rho_{\mathrm{f}}}{d(1-\phi)^{3.1}}\left(\frac{3}{10}\left|u_{\mathrm{f}}-u_{\mathrm{p}}\right|+18.3 \frac{\eta_{\mathrm{f}}}{\rho_{\mathrm{f}} d}\right)
$$

To complete the system equations, closure models for both the fluid and particle shear stress should be supplemented. For turbulent liquid flows, the fluid shear stress can be described by a simple classical turbulent closure based on the mixing length theory (Berzi and Jenkins (2008)) as:

$$
\tau_{x z, \mathrm{f}}=-(1-\phi) \rho_{\mathrm{f}}\left(v_{\mathrm{f}}+v_{\mathrm{t}}\right) \frac{\partial u_{\mathrm{f}}}{\partial z}
$$

where $v_{\mathrm{t}}$ is the turbulent viscosity modeled by $v_{\mathrm{t}}=l^{2} \partial_{\mathrm{z}} u_{\mathrm{f}}$. The spatial length $l$ is the mixing length and is proportional to the distance from the flow base ( $l=\kappa z$, where $\kappa$ is the von Karman constant) for a turbulent liquid flow free of particles. The presence of particles is however expected to influence the turbulence and thus to modify the mixing length. In absence of any experimental data, the simplest approximation is to use the law (2.18). However, more elaborate models have been developed to account for the influence of particles on fluid turbulence (Jenkins and Hanes (1998), Hsu et al. (2003, 2004), Cantero et al. (2012)).

The closure for the particle phase is another crucial issue which is not yet solved. Depending of the flow regime, different closure models have been proposed.

(i) For collisional flows of heavy grains (called sheet flows), where collisional dissipation dominate viscous dissipation (Jenkins and Hanes (1998)), the particle shear stress can be derived from the kinetic theory of granular gas (see section 2.3.1). The particle pressure and the particle viscosity are thus given in terms of the granular temperature. This simple model has the capacity to predict profiles of particle concentration and particle mean velocity in steady and fully developed sheet flows.

(ii) For dense liquid particle mixture, several recent approaches employing the " $\mu(I)$ " rheology for the particle shear stress closure (cf. section 2.3.2) have been developed by Cassar et al. (2005). For dense viscous granular suspension, a more refined relation has been proposed by Boyer et al. (2011). According to the flow regime (inertial or viscous), the pertinent inertial parameter to be considered is respectively $I_{\mathrm{pi}}$ and $I_{\mathrm{v}}$. In this context, Berzi and Jenkins (2008) have considered the problem of steady and fully developed flows of particles and fluid down a slope. They made the assumption that the fluid rheology is described by an eddy viscosity model (cf. (2.18)) and the granular phase by a shear-ratedependent friction law $\mu(I)$. They investigated the case where the particle inertia dominates the fluid drag (i.e., particle inertia-dominated regime). In other words, they assumed that $I=I_{\mathrm{pi}}$. This approach was shown to be successful for predicting quantitatively the velocity and density profiles observed in the experiments of Armanini et al. (2005) in which a mixture of fluid and particles is recirculated through an inclined channel. This approach is restricted to steady and fully developed flows and therefore disregard dilatancy effects.

For describing bed load transport in laminar flows, Aussilous et al. (2013) have implemented a two-phase continuum model using a shear-rate-dependent friction law $\mu\left(I_{\mathrm{v}}\right)$ for the granular phase and a volume-fraction-dependent viscosity for the fluid phase. This approach provides reasonable predictions for particle velocity and particle density profiles.

2.4.5 Unsteady flows. We shall now discuss the capability of the two-phase approaches to model the transient dynamics during which the mixture can contract or dilate.

During the transient, part of the relative vertical displacements between the fluid and the granular phase are caused by the dilation and compaction of the granular material.

These displacements affect the pressure field. The classical approach is to split the fluid pressure into two contributions: $P_{\mathrm{f}}=P_{\mathrm{f}}^{\mathrm{h}}+P_{\mathrm{f}}^{\mathrm{e}}$, where $P_{\mathrm{f}}^{\mathrm{h}}$ is the hydrostatic fluid pressure, verifying $\nabla P_{\mathrm{f}}^{\mathrm{h}}=\rho_{\mathrm{f}}$ g and $P_{\mathrm{f}}^{\mathrm{e}}$ is the excess pore-fluid pressure.

The key issue is thus to provide a closure relation between the particle volume fraction and the excess pore pressure. Iverson (1997) argued that it is reasonable to assume that the particle volume fraction is a function of only the excess pore pressure and that the change in particle volume fraction due to changes in excess pore pressure can be expressed in terms of the compressibility coefficient 
$K=\partial \phi / \partial P_{\mathrm{f}}^{\mathrm{e}}$ of the sediment-water mixture. Within this hypothesis, the equation for the particle volume fraction reduces to a standard diffusion equation that can readily be solved.

Other closure equations can be found in the literature. For example, Pailha and Pouliquen (2009) consider an equation relating the relative variation of the particle volume fraction to the shear-induced dilatancy angle. More precisely, their model is inspired from the critical state theory proposed by Roux and Radjai (1998) and is based on the concept of the dilatancy angle $\psi$, which gives the rate of dilation (compaction) of the material under shear. Based on this theory, the closure equations for the particle shear stress and pressure are modified and rewritten as:

$$
\begin{aligned}
& \tau_{x y, \mathrm{p}}-\tau_{x y, \mathrm{p}}^{\mathrm{eq}}=\tan \psi P_{\mathrm{p}} \\
& \phi-\phi^{\mathrm{eq}}=\frac{\tan \psi}{K}
\end{aligned}
$$

supplemented by

$$
\frac{1}{\phi} \frac{d \phi}{d t}=\tan \psi \dot{\gamma}
$$

where $\dot{\gamma}$ is the local particle shear rate. $\tau_{x y, \mathrm{p}}^{\mathrm{eq}}$ and $\phi^{\mathrm{eq}}$ are the stress and the volume fraction in the steady regime (Pailha and Pouliquen (2009)). This model represents the simplest shear-rate dependent critical state theory for granular/fluid mixtures and has been used successfully to model submarine avalanches (cf. next section).

\subsection{Boundary conditions}

The physical principles on which the equations of continuum models are based are the balance laws of mass and momentum (and energy) and constitutive closure equations describing the mechanical behaviour of the material involved. These equations are then used together with boundary conditions to predict the motion of the system.

The behaviour of granular flows contrasts with the one of single phase newtonian fluids as the former depend crucially on the nature of the boundaries (i.e., the properties of the base atop which the flow occurs and the possible presence of lateral confinement). Most experimental and numerical studies generally consider bumpy, flat frictional or erodible basal boundaries. Bumpy boundaries usually consist of particles glued on a flat substrate. In most studies, glued particles are the same as those of the flowing material. However, the bumpiness can be increased or decreased by using grains larger or smaller than the flowing grains. The spatial repartition of the grains can also be used to modify the bumpiness of the boundary.

The conditions at the boundaries, for granular flows, differ from the one used for Newtonian fluids. For the latter, no-slip conditions at solid boundaries is often achieved. In contrast, a granular flow slips relative to a boundary; so, in addition to collisional dissipation at the boundaries, fluctuation energy can be generated by slip (Richman (1988)). Consequently, boundaries can either provide or remove fluctuation energy from the flow.

Establishing ab initio boundary conditions for granular gases is complex: in addition to the difficulty of solving the Boltzmann equation near a solid boundary, one has to know the properties of the collision between the particle and the boundary. The feed-back effect of the boundary on the distribution function of the incoming particles must be taken into account. Approximate boundary conditions at solid surfaces describing the way energy fluctuation is provided to the flow can be applied to flows down bumpy inclined (Richman and Marciniec (1990), Jenkins (1994), Delannay et al. (2007), Kumaran (2015)).

Within the framework of the $\mu(I)$ rheology, boundary conditions also cause difficulties. For example, the effect of changing the degree of bumpiness of a bumpy base is not clear: does it only modify the shear and velocity in a boundary layer? Or does it change the whole bulk rheology (Goujon 
et al. (2007)). It has been shown that relatively small changes at the boundary can induce transitions from disordered to ordered flow states (Silbert et al. (2002), Kumaran and Maheshwari (2012), Kumaran and Bharathraj (2013)). The slip velocity at the boundary (flat or, to a lesser extent, bumpy) should be related to the physical characteristics of the grains and the boundary (Artoni et al (2009), Artoni et al. (2012)).

Free surface flows induce other questions concerning boundary conditions. Phase transition from liquid to gas may occur at the free surface. As the pressure decreases, the number $I$ becomes very large and the kinetic regime appears. This transition is neither predicted nor described by the $\mu(I)$ rheology. Another transition also appears in free conditions: as will be seen in section 3.3, unconfined flows can create lateral levées, which correspond to erodible boundaries. It can be seen as a jamming/unjamming transition. The same behaviour is also observed on very dissipative bases (Louge et al. (2015)), and when there is the spontaneous formation of a heap, called SSH (see section 3.2). The flowing "liquid" zone is in contact with a quasi-static part where the displacement of the grains decreases exponentially. The number $I$ vanishes when approaching this part. The $\mu(I)$ rheology does not describe this quasi-static regime, which probably requires a non-local rheology, as already mentioned (section 2.3.2).

In the case of a flow over an erodible base composed of the same material, different approaches have been developed to describe the erosion/deposition process. One of them is the BCRE model (Bouchaud et al. (1994), Boutreux et al. (1998)). Assuming a thin flow on a pile slope close to the angle of repose, this model provides a continuum phenomenological description of the surface dynamics by two variables: $b(x, t)$, the local height of the pile (profile of immobile grains) and $h(x, t)$, the thickness of flowing grains. These variables are dynamically coupled by an interaction term $\Gamma(b, h)$ allowing conversion from flowing to static grains and vice versa (deposition/erosion). The mass conservation is then expressed as:

$$
\begin{aligned}
& \frac{\partial b}{\partial t}=-\Gamma \\
& \frac{\partial h}{\partial t}+U \frac{\partial h}{\partial x}=D \frac{\partial^{2} h}{\partial x^{2}}+\Gamma
\end{aligned}
$$

where $U$ is the constant drift downhill velocity of the rolling grains, and $D$ is a constant diffusion coefficient. To close the model, a phenomenological relation between the interaction term $\Gamma$ and the local slope $\theta$ has to be written. A first approach is to set the amplitude of exchange rate with the distance to a neutral inclination angle $\theta_{\mathrm{n}}: \Gamma=\gamma_{c} R\left(\theta-\theta_{n}\right)$ so that if $\theta>\theta_{\mathrm{n}}$ erosion is dominant (else it is deposition), $\gamma_{c}$ is the collision frequency of a mobile grain with the static phase. When $h$ is thicker than a few grain diameters $(h>\lambda)$ the previous exchange term is not realistic, grains from the upper layers do not interact with the static phase (Boutreux et al. (1998)): $\Gamma=v_{\text {up }}\left(\theta-\theta_{n}\right)$, where $v_{\text {up }}$ is a constant corresponding to $\gamma_{c} \lambda$. In their recent review on granular surface flows, Iverson and Ouyang (2015) have listed the alternative formulations of the exchange rate term that have been proposed in the literature. We shall point out that this simple phenomenological model can be formally derived from hydrodynamic models based on Saint-Venant equations (e.g., Gray (2001), Aradian et al. (2002), Bouchut et al. (2008)). However, as highlighted in Bouchut et al. (2008), the energy balance of these models is not always consistent. More refined descriptions of erosion and deposition processes can be developed from the knowledge of the constitutive law of the granular fluid coupled with a yield criterion without additional phenomenological law for the erosion rate (Bouchut et al. (2016), Lusso et al. (2015a)). The exchange rate cannot be derived explicitly except in very simple configurations.

For fluid particle mixture continuum models, the same difficulties arise for accounting properly for what happens at the boundaries and for deriving appropriate boundary conditions. Recently, an interesting approach has been introduced by Ancey and Heyman (2014) as a microstructural model of bedload transport, which describes the advection and scattering of coarse particles carried by a turbulent water stream down a sloping granular bed. Fluctuations of the particle flux are generated by particle exchanges with the bed consisting of particle entrainment and deposition. The evolution of the 
number of moving particles is described probabilistically using a coupled set of reaction-diffusion master equations.

Another key issue in fluid particle mixture continuum models is to describe what happens at the free surface. In particular it is important to describe different free surfaces for the solid and fluid phases to make it possible for the fluid to be sucked or expelled from the granular material when dilatancy effects are accounted for (Bouchut et al. (2015b)).

\subsection{A simplified model: depth averaged approach}

Practically, solving the mass and momentum conservation full set of equations requires a prohibitive computational cost when applied to natural flows over real topography and simplifications are necessary.

A first approximation is to assume that the flow is thin compared to its extent along the slope (i. e. small aspect ratio). This is the case for most geophysical flows that are a few meters thick and travel distances of several hundred meters to several kilometers. Asymptotic development of the equations based on this thin-layer approximation makes it possible to neglect some terms such as the vertical acceleration, generally leading to the hydrostatic pressure assumption. Thin layer models solving the resulting simplified $2 \mathrm{D}$ or 3D equations have been proposed based on the partial fluidization method that takes into account an order parameter describing the state of the granular matter from static to flowing (Aranson and Tsimring (2002), Mangeney et al. (2007b), Aranson et al. (2008)) or by using visco-plastic models with a yield stress separating static and flowing zones (Bouchut et al. (2016), Lusso et al. (2015a)).

Assuming thin-layer flows together with averaging the equations over the depth of the flow makes it possible to further reduce the computational cost as proposed in the pioneering work of Savage and Hutter (1989) partially based on former german (Voellmy (1955)) and russian (Grigorian et al. (1967)) works on snow avalanches (Figure 4). In that case, the whole granular material is assumed to be flowing. The resulting system (so-called Saint-Venant or shallow-water) involves two unknowns, the thickness and the mean (depth-averaged) velocity of the flowing mass. This hydrodynamic model is widely used to simulate natural flows. The first order asymptotic development leads essentially to a hydrostatic pressure (including centrifugal forces) even though attempts have been made to include the vertical acceleration (Denlinger and Iverson (2004)). The mass and momentum conservation equations in the simplest $1 \mathrm{D}$ case (i. e. flow over a $2 \mathrm{D}$ topography $b(x)$ ) then read

$$
\begin{aligned}
\frac{\partial h}{\partial t}+\frac{\partial}{\partial X}(h \bar{u}) & =0 \\
\frac{\partial}{\partial t}(h \bar{u})+\frac{\partial}{\partial X}\left(h \bar{u}^{2}\right) & =g h \sin \theta+\frac{\partial}{\partial X}\left(g \cos \theta \frac{h^{2}}{2}\right)-\mu h\left(g \cos \theta+\frac{\bar{u}^{2}}{R}\right) \frac{\bar{u}}{|\bar{u}|}
\end{aligned}
$$

where $\bar{u}(X, t)$ is the depth-averaged velocity in the downslope direction $X$ (see Figure 4) and $|\bar{u}|$ it's absolute value, $h(X, t)$ is the flow thickness, $\theta(X, t)$ the slope angle, and $R(X, t)$ the curvature radius of the topography. In that case, the momentum equation reduces to the balance between the acceleration, the gravity force, the force related to the pressure gradient and the friction force including centrifugal acceleration effects.

Formally, the friction terms derived from the Coulomb friction law based on this depth-averaged asymptotic method appear both in the basal shear stress and in the pressure gradient term when anisotropy of normal stresses is taken into account (e. g. Gray et al. (1999), Denlinger and Iverson (2004)). However, because accurate development of the thin-layer approximation for a Coulomb friction law has never been done over a 3D arbitrary topography and because anisotropy effects have been questioned for granular flows (e. g. Ertas et al. (2001)), the models generally only consider the term related to the basal shear stress to describe the friction dissipation (e. g. Mangeney-Castelnau et al. (2005)). As a result, these models loose the relative importance of the sliding and shearing contributions to the flow. Consequently, one of the key issues of the method is the modelling of the averaged friction dissipation at the base. The basal friction is generally described phenomenologically 
in the framework of Coulomb friction using friction coefficient which may be constant or depend on the inertial number (see for example Pouliquen and Forterre (2002), Mangeney-Castelnau et al. (2003), Mangeney et al. (2007a), Pirulli and Sorbino (2008)). Note, however, that fitting the basal friction dissipation (the only dissipation term in the model) to reproduce deposit extent of laboratory or field scale events result implicitly in an empirical quantification of the whole effective friction within the flow (Lucas et al. (2014)).

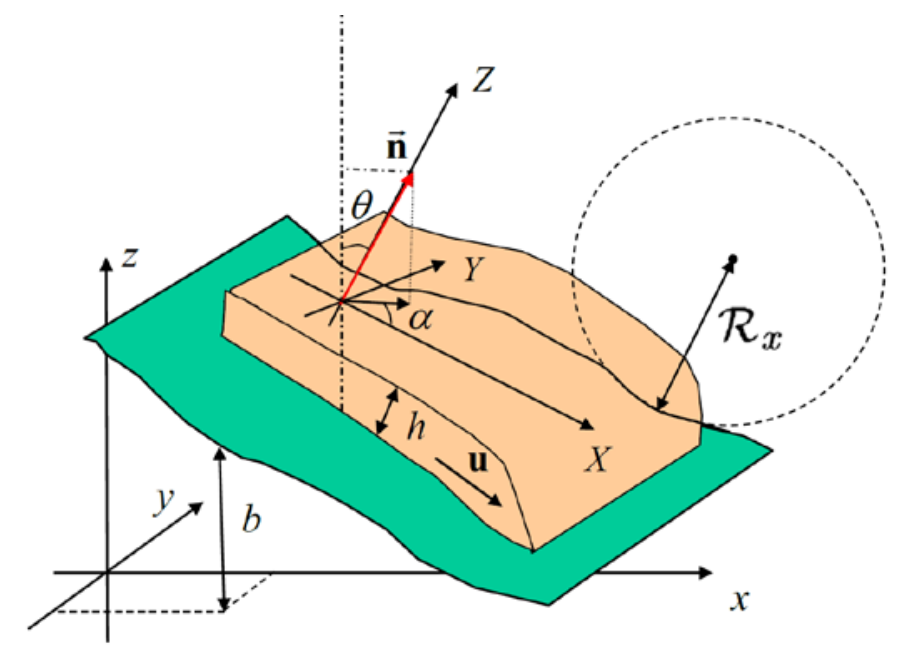

Figure 4. 2D depth-averaged thin-layer continuum model where the depth-averaged velocity field $\overline{\mathbf{u}}(x, y, t)$ and the flow thickness $h(x, y, t)$ are calculated at time $t$. Note that the velocity is averaged in the $Z$-direction, where $(X, Y, Z)$ is the variable reference frame linked to the topography $b(\mathrm{x}, \mathrm{y})$ and $\theta$ is the steepest slope angle. The curvature of the topography is a tensor and we represent here one of its component $\mathrm{R}_{X}$ (see Appendix A of Mangeney et al. (2007a)).

One of the main difficulties in deriving thin-layer depth-averaged models is to appropriately take into account topography effects, which is a key issue for application to natural landslides. Indeed, these flows are thin in the direction perpendicular to the flow and not in the vertical direction (Figure 4). For these reasons, a series of asymptotic developments have been performed in a variable reference frame linked to the topography for complicated but specific topographies (see Pudasaini et al. (2007) for a review). By considering a fixed reference frame but applying the thin-layer approximation in the direction perpendicular to the slope, Bouchut et al. (2003) and Bouchut and Westdickenberg (2004) derived a model that takes into account accurately all the curvature terms for flows over an arbitrary topography, including centrifugal forces ignored in other models (see Appendix A of Mangeney et al. (2007a)). These terms can be important in the dynamics of natural landslides (Favreau et al. (2010), Moretti et al. (2015)).

By assuming that the whole material flows, the Savage-Hutter model fails to capture the features of flows for which interaction with an erodible base prevails. This is the case for example for dambreak granular flows (see section 3.4). Indeed the transition between static and flowing regions in a depth-averaged model requires the determination of an equation for the evolution of the moving interface between the flowing and non-flowing parts, an interface that is not always well defined. As discussed in section 2.5, this point is currently a matter of debate, notably because a quantitative study of the erosion/deposition rate at the grain scale is still missing. Moreover, the proposed approaches are generally overly schematic to be extended to natural flows over real topography (Bouchut et al. (2008)). A key new approach using the depth-averaged $\mu(I)$-rheology and prescribed Bagnold velocity profiles (Gray and Edwards (2014)) explicitly model the formation of erosion-deposition waves (Edwards and Gray (2015)), where completely stopped regions can form between mobile wave crests. A depth-integrated model taking into account a linearization of the $\mu(I)$-rheology and prescribed Sshaped velocity profile was also proposed by Capart et al. (2015). This model reproduces granular flows over erodible beds. However, by prescribing a specific shape of the velocity profiles, these models cannot reproduce the different profiles observed in highly transient flows. A new alternative 
approach based on mutlilayer shallow models has been recently proposed that makes it possible to calculate the different velocity profiles within the shallow granular layer and thus the static/flowing transition (Fernandez-Nieto et al. (2016)).

The depth-averaged approach has been also used for modeling particles/liquid mixtures. In particular, depth-averaged mixture models and two-phase models have been developed by Iverson and Denlinger (2001), Denlinger and Iverson (2001) and Pitman and Lee (2005), Pelanti et al. (2008) respectively. In these versions of the two-phase flow equations, the flowing layer is assumed to be thin. This model allows describing, within a tractable approach, unsteady and non-uniform configurations encountered in real geophysical flows. In these works, the granular phase is described as a Coulomb material but dilatancy is disregarded. Bouchut et al. (2015a) showed that the constitutive equation required to close the system of two-phase flows has been replaced by two free surface boundary conditions in Pitman and Lee (2005), and Pelanti et al. (2008), leading to a non-dissipative energy equation for the resulting model. This problem can be solved by prescribing the incompressibility of the solid phase.

The coupling between the dilatancy of the granular layer and the pore pressure was taken into account in depth-averaged mixture models by Iverson (1997, 2005). In this approach, Iverson explicitly incorporates an equation of the pore fluid pressure as discussed earlier. The landslide is described as a rigid block sliding down a slope, the source of dilatancy being localized at the base. The pore pressure builds up at the base and diffuses through the granular phase which is assumed to behave as a poro-elastic medium. The limit of this approach is that the long term evolution and the fully developed regime cannot be described because no shear rate dependence is incorporated in the rheology of the granular phase. Later on Iverson and George (2014) incorporated the shear rate dependence.

Pailha and Pouliquen (2009) combine the depth-averaged two-phase approach with the rheogical model for fluid/granular mixtures complemented by the critical state theory by Roux and Radjai (1998). This model is able to describe both the dilatancy effects experienced by the granular skeleton during the initial deformations and the rheology of wet granular media when the flow is fully developed. The predictions of the model agree quantitatively with the experiments by Pailha et al. (2008) who investigated the role of the initial volume fraction on the dynamics of underwater avalanches. However, this model assumes uniform flow along the slope aligned direction. The extension of this model to non-uniform flow in the framework of thin-layer depth-averaged flows is proposed in Bouchut et al. (2015b). Following Iverson et al. (2000), these models assume that, when dilation occurs the fluid is sucked into the granular material, the pore pressure decreases and the friction force on the granular phase increases. On the contrary, in the case of contraction, the fluid is expelled from the mixture, the pore pressure increases, and the friction force diminishes. In order to enable the fluid to enter or exit the mixture, Bouchut et al. (2015b) proposed a two-layer model with a fluid layer on top of the two-phase mixture layer.

The description of submarine granular flows and generated water waves should combine the description of the two-phase granular flows and its interaction with the water column. Up to now, these processes are described by oversimplified approaches that decouple the submarine flow from the water motion (Le Friant et al., 2003) or by two-layer depth-averaged models that account explicitly from the avalanche/water interaction but without describing the dilatation/compression processes within the granular flow (Fernandez-Nieto et al. (2008)). Furthermore, in that case, the thin-layer approximation should be imposed in the downslope/normal direction for the flowing avalanche while it should be imposed in the horizontal/vertical direction for the description of generated water waves.

Other physical processes such as polydispersity and related granular segregation have been introduced in thin-layer depth-averaged models, successfully reproducing key experimental observations (e. g. Gray and Thornton (2005), Gray and Kokelaar (2010), Gray and Ancey (2011), Wiederseiner et al. (2011), van der Vaart et al. (2015)). As landslide characteristics change during time even for a single event, one of the challenges is to incorporate a fluid phase as well as erosion/deposition, fragmentation and segregation processes within a single model.

\subsection{An alternative: discrete approach}

Developed in the late 70's (Cundall and Strack (1979)), Discrete Element Modeling (DEM) may be seen as an outgrowth of molecular dynamics simulations used in statistical physics. In the past three 
decades, it has been widely used to study flowing or static granular media. It considers individual grains (usually, for simplicity's sake spheres or polyhedra) interacting through frictional collisions and/or enduring contacts. Interactions between grains are approximated by idealized force models that dissipate energy (see below). The position and velocity of each grain are obtained by integrating Newton's equations of motion. Relevant transport quantities and bulk properties as the velocity field, stress tensor, or local packing fraction can then be computed. Contrary to molecular systems, dissipation is an important characteristic of granular systems. It is thus necessary to employ realistic approximations to model energy loss in interacting grains. To that end, there are essentially two basic approaches: 'Hard' Particle Models - Contact Dynamics (CD) and Event-Driven (ED) - which consider grains to be infinitely stiff, and 'Soft' Particle Models (SPM) for which overlapping grains interact at compliant elastic, frictional contacts with dissipative elements providing inelasticity. In the latter case, the interaction is a function of an allowed overlap between colliding grains that model the contact deformation. As an example, the normal force $F_{n}$ between contacting grains can be approximated by the association of a spring of normal stiffness $k_{\mathrm{n}}$ and a dashpot of normal damping coefficient $\gamma_{\mathrm{n}}$ :

$$
F_{\mathrm{n}}=k_{\mathrm{n}} \delta+\gamma_{\mathrm{n}} \frac{\mathrm{d} \delta}{\mathrm{d} t}
$$

where $\delta$ is the grain overlap. In the presence of friction and contrary to the CD method (see below), the Coulomb law has to be regularized to avoid the discontinuity at zero sliding velocity. As an example, the tangential force can be modeled by a tangential spring

$$
F_{\mathrm{t}}=k_{\mathrm{t}} u_{\mathrm{t}}
$$

where $u_{\mathrm{t}}$ is the tangential displacement at the contact whose magnitude is truncated to satisfy the Coulomb law $\left|F_{\mathrm{t}}\right| \leq \mu\left|F_{\mathrm{n}}\right|$, where $\mu$ is the friction coefficient (Cundall and Strack (1979)).

Contact Dynamics (CD), by considering the grains as perfectly rigid bodies, neglects grain deformation. Thus, deformation of the granular medium is exclusively due to grain rearrangements. The volume exclusions of the perfectly rigid grains are formulated by unilateral constraints: the gap between grain surfaces must be larger or equal to zero. Consequently, the number of degrees of freedom depends on the number of aforementioned constraints and thus on the number of contacts that may change with time. For frictional grains, a second type of constraint has to be taken into account: the non-sliding condition if the Coulomb yield threshold is not satisfied. The contact laws are nonsmooth, as they express no functional dependence between relative velocities and contact forces. At each time step, iterative methods are used to determine both forces and velocities. The time step should be chosen much smaller (typically 100 times smaller) than the characteristic time of the system (e.g. the inverse of the shear rate). By contrast, in SPM simulations, the number of degrees of freedom does not change in time and integrating the equations of motion requires a time step that is small enough to resolve the grain deformations during collisions. Thus, the time steps used in SPM are generally smaller than those used in $\mathrm{CD}$, leading to larger computational times.

The Event-Driven method (ED) consists in advancing the system from event to event by repeating the following steps: (i) predict the next collision event between two grains, (ii) bring forward the system to that instant, and (iii) compute the velocities after the collision. This method is very precise because the grain dynamics can be solved analytically between collisions. It is also efficient as the effective time step matches the mean free time. Unfortunately it assumes binary collisions and is thus not suitable to simulate enduring contacts. Therefore ED is relevant to model collision-dominated flows but not systems where enduring contacts prevail. In the latter case, SPM and CD are more suitable.

DEM simulations mimic laboratory experiments. There are advantages of these simulations over experiments since they permit the measurement of any physical quantity without perturbing the system. Moreover, all parameters such as size and mass distribution, friction properties, grain restitution coefficient and eventually rigidity (i.e. Young modulus) can be tuned independently. For example, one can freeze the grain rotation or use perfectly elastic grains, which is difficult, if not 
impossible in the lab. The attractiveness of these simulations is thus obvious but their ability to reproduce laboratory experiments (i.e. a large number of grains over a long period of time) is recent. This is mainly due to the large amount of computational time needed to carry out such simulations. Simulating a natural avalanche in reasonable times is still impossible because of (i) the very large number of grains involved (several millions or billions) and (ii) their wide and generally unknown disparity of properties, which reduces the computation efficiency. However, it is an inescapable tool at laboratory scale.

DEM can be coupled with a continuum fluid model to describe fluid/particles flows. These hybrid methods have been developed recently for modelling bedload transport in rivers or aeolian sand transport in deserts (Carneiro et al. (2011), Duran et al. (2012)) and can be in principle used for describing debris flows.

\section{Experimental results and their simulation}

We focus here on experiments that are very simple compared to geophysical granular flows. Large scale experiments involving length-scale of tens of meters and real debris flow material are closer to natural flows but are less reproducible and make it difficult to separate the effects of the different processes involved. On the other hand meter-scale experiments involving simpler granular material (beads, sand, etc.) provide useful framework to identify the effects of individual mechanisms and for their modelling, even though the relative importance of the different processes may be very different compared to natural situation at this scale.

\subsection{Unjamming transition}

The existence of the unjamming transition is fundamental in controlling the behaviour of granular materials. The latter are jammed at rest and can sustain some load, but if a threshold shear stress is exceeded, part of the material starts to flow. In nature, geological processes like landslides or rock avalanches involve an unjamming transition of granular media.

For free surface flows of granular systems driven by gravity, the unjamming transition above a critical shear stress is evidenced by the existence of an angle of maximum stability of a pile: $\theta_{\mathrm{a}}$. It is the angle at which the flow starts; the angle of the pile relaxes then towards the smaller angle of repose $\theta_{\mathrm{r}}$. Parameters like humidity, system dimensions, friction between grains, bottom roughness, and packing fraction can influence the value of $\theta_{\mathrm{a}}$. Many studies have been devoted to these angles, most of them focusing on the succession of avalanches in a rotating drum (Fischer et al. (2008), GDR MiDi (2004), Courrech du Pont et al. (2003a, 2003b)), or on a continuously fed pile (Frette et al. (1996), Jaeger et al. (1989)). An alternative method for investigating the unjamming transition is to incline gently in the gravity field an initially horizontal box filled with grains (Aguirre et al. (2000, 2001), Boltenhagen (1999), Kiesgen de Richter et al. (2012)), or a plane on which has been prepared a granular layer of uniform thickness $h$ (GDR MiDi (2004), Pouliquen (1999)). The grains start flowing when the plane inclination reaches a critical angle $\theta$ depending on the layer thickness $h$. The thickness then slowly decreases; the material slows down, stops, and leaves a static layer of material on the bed of thickness $h_{\text {stop }}(\theta)$. By starting with different thickness $h$ of the initial layer the curve $h_{\text {stop }}(\theta)$ can be obtained. It divides the phase diagram $(h, \theta)$ in a region where no flow is possible $\left(h<h_{\text {stop }}\right)$ and a region where flow may occur $\left(h>h_{\text {stop }}\right)$. That critical thickness $h_{\text {stop }}$, which is influenced both by the bulk material and by the bumpiness of the incline (Goujon et al. (2003)), decreases with the inclination angle (see Fig. 5): $\theta_{1}$ corresponds to the angle where $h_{\text {stop }}(\theta)$ diverges, its value is generally close to the angle of repose of the corresponding static pile; $\theta_{2}$ corresponds to the angle where $h_{\text {stop }}(\theta)$ vanishes. A similar phase diagram was obtained in numerical simulations (DEM) where periodic boundary conditions are imposed both along the flow direction and in the transverse direction. The bumpy bottom was made of fixed particles (Silbert et al. (2001)).

\subsection{Steady and fully developed flows down inclines}

We will first focus on steady and fully developed flows, referred in the remaining of the paper as SFD flows, i.e. flows whose quantities, such as the flow height, mass flow rate $Q$, etc. , change neither with time (steady) nor with the position in the main flow direction (fully developed). Such systems have yielded a wealth of information useful to understand geophysical flows although the latter are generally unsteady and not fully developed. For sake of simplicity, physicists first focused on dry and 
nearly monosized grain flows which have two major advantages: (i) segregation is weak or absent and (ii) the effect of the surrounding and interstitial fluid can be neglected as long as grains are large enough. Despite this simple configuration, the observed regimes are numerous and the nature of the boundaries (flat, bumpy or erodible base and sidewalls, etc.) is of crucial importance.

Most of the studies of granular gravity-driven flows on inclines reported in the literature deal with flows of grains on bumpy surfaces, both in experiments and in DEM numerical simulations (GDR MiDi (2004), Delannay et al. (2007) and references therein). Such a configuration tends to avoid the inherent discontinuity and sliding observed at a flat base by gluing on the surface of the incline grains of the same nature as those involved in the flow. In these conditions a no-slip boundary condition at the base of the flow is observed, at least for moderate inclination angles. The use of very wide channels in most experiments is an attempt to avoid the influence of confinement due to the lateral boundaries (e.g. sidewalls). For the same purpose, most numerical simulations use periodic boundary conditions in the transverse direction.

One of the main observations for steady and fully developed flows down "unconfined" bumpy inclines is their limited domain of existence. They have indeed been observed only in a finite range of inclinations and thicknesses. As seen above, the flowing region is bounded by the $h_{\text {stop }}(\theta)$ curve in the phase diagram $(\theta-h)$. Moreover, a too thick layer and/or a too inclined plane are supposed to lead to accelerated flows (GDR MiDi (2004), Pouliquen (1999), Silbert et al. (2001)). However, it is worth noting that the experimental attainment of SFD flows is restricted by the physical length of the chute: a flow may require a length longer to that of the incline to reach its SFD state. Thus the measurement of the maximal angle above which an "accelerated regime" is observed may depend on the setup used (Brodu (2013)). Numerical simulation are subject to the same kind of restriction as the runs are limited in time and the above mentioned maximal angle may depend of this time.

Unidirectional SFD flows possess some noticeable properties (Pouliquen (1999), Silbert et al. (2001), GDR MiDi (2004)):

(a) the volume fraction is constant across the depth of the flow -and independent of the value of this depth- except for the boundary layers near the bottom and the surface;

(b) for thick flows, the velocity follows the so-called Bagnold profile i.e. it varies with the depth to the power $3 / 2$;

(c) for thin flows, when $h$ is slightly larger than $h_{\text {stop }}$ (and thus $\theta$ slightly larger than $\theta_{1}$ ), the velocity profile is close to linear (Rajchenbach (2003), Silbert et al. (2001));

(d) the Froude number defined by $\bar{u} /(g h)^{1 / 2}$, where $\bar{u}$ is the depth averaged velocity, is empirically found to be proportional to the ratio $h / h_{\text {stop }}$. This is consistent with the claim that when $h_{\text {stop }}=0$ (i.e. $\theta$ $>\theta_{2}$ ), the velocity diverges (accelerating flows).

The inclined-plane geometry has an interesting property: for SFD flows, if sidewall friction is negligible, the tangential and normal forces exerted on the base correspond exactly to the components of the flow weight. More generally, if we consider a material slab of arbitrary thickness and bounded by the free flow surface, the tangential and normal forces on the base of the slab correspond also exactly to the components of the slab weight. Their ratio, the effective friction coefficient, is thus always equal to the tangent of the angle of inclination: $\tan \theta$. In the frame of the $\mu(I)$ rheology described previously (section 2.3), it means that, for flows which are both SFD and invariant in the direction transverse to the flow, the inertial number is uniform in the flow and solely function of the inclination angle. It is thus easy to show that, for those flows, the Bagnold profile results from the $\mu(\mathrm{I})$ rheology (GDR MiDi (2004)). The number $I$ can then be related to $h_{\text {stop }}(\theta)$ through the aforementioned linear relationship between the Froude number and the ratio $h / h_{\text {stop }}$. The measure of the number $I$ for an inclination angle $\theta$, gives the function $\mu(I)$, as $\mu=\tan \theta$. Note that there is no reason why a SFD flow should not vary in the direction transverse to the flow. If it varies, neither the local ratio of shear to normal stress, nor the inertial number, is expected to remain uniform.

Up to this point, the overall situation appears to be simple, with a limited domain of the parameter space $(\theta-h)$ in which unidirectional Bagnold type dense SFD flows occur. As expected, when increasing the inclination angle $\theta$, these flows become unstable. Non-transverse invariant stripe state with spanwise vortices emerges naturally from their destabilization (Börzsönyi et al. (2009)). Nevertheless, this new state is also SFD, and, as explained before (section 2.3.2), the 3D extension of the $\mu(I)$ rheology is not able to describe it. 
Besides these studies on flows in wide channels (where side-wall friction is neglected), extensive experimental works have shed light on the crucial influence of sidewalls (Taberlet et al. (2003, 2004, 2008), Bi et al. (2005, 2006), Jop et al. (2005), Richard et al. (2008), Brodu et al. (2013, 2015)) on the flow behaviour. The so-called Sidewall-Stabilized Heap (SSH) is a spectacular effect: at any given inclination angle, above a critical flow rate, a static pile forms along the incline. The pile, whose angle increases with the flow rate, is stabilized by the flow atop it (Taberlet et al. (2003)). If the flow rate is constant in time, the flow atop this sidewall-stabilized heap is SFD. The influence of sidewalls on SFD flows on top of a static pile has been studied by carrying out experiments in setups of different widths, up to 600 particle diameters (Jop et al. (2005)).

The properties of flows occurring atop a SSH are different from that observed for SFD flows over bumpy inclines. For instance (i) the main-flow direction velocity profile is no more concave but convex, (ii) the volume fraction varies from zero to the random close packing with a characteristic length that scales with the tangent of the angle of the SSH and thus with the flow rate. Consequently, the gas, liquid and solid behaviours of granular systems are present at the same time in such systems and the corresponding rheology is much more complex than that observed for negligible confinement, especially at high flow rate and/or important confinement. Therefore, although the application of the $\mu(I)$ rheology to such flows could remain valid for regions that are neither quasi-static nor too rapid (Jop et al. (2006)) or for moderate confinement and flow rates, it is unable to capture the full behaviour of the flow in the general case (Jop (2015)). Moreover, in a similar context of confined surface flows, but not SFD, we have seen (section 2.3.2) that the 3D extension of the $\mu(I)$ rheology failed.

Surprisingly relatively few experimental studies (Savage (1979), Johnson et al. (1990), Ahn et al. (1991), Louge and Keast (2001)) have considered the case of flat frictional inclines. In contrast with a bumpy plane, the mean-flow direction velocity profile involves a considerable slip velocity at the base (Ahn et al. (1991), Artoni et al. (2012)). In their experiments, Louge and Keast (2001) observed SFD flows only for a given range of inclination angles $\left[\theta_{\min }, \theta_{\max }\right]$, independently of the flow height. Here also, the experimental attainment of SFD flows is restricted by the physical length of the chute. Nevertheless, if sidewall friction is negligible, it can be deduced from the force balance that an upper bound exists for $\theta$, its tangent is lower than the friction coefficient between the flat incline and a grain (Brodu et al. (2015)). The case of $\theta_{\min }$ is more complex and its value, when it is defined, depends crucially of the confinement (Brodu et al. (2013)).

Compared to the bumpy incline configuration, flows on flat frictional surfaces involve a much faster overall velocity, due to the presence of a basal layer of rolling grains, upon which slides the bulk of the unidirectional flow. The bottommost layer of grains is then an effective base for the flow bulk, which follows the aforementioned Bagnold scaling (Delannay et al. (2007), Artoni et al. (2012), Brodu et al. (2013)). The analogy with an effective bumpy base extends to the presence, at larger inclination angle, of a convective regime with similar velocity and density profiles. The $\mu(\mathrm{I})$ rheology is valid in the case of unidirectional flows but its use in the case of convective regime is more problematic (Brodu et al (2013)).

Recent extensive numerical works (Brodu et al. (2015)) studied flows down flat frictional inclines at high angle of inclination and/or flow rate, in the presence of sidewalls. They show that the system can achieve numerous steady and fully developed regimes which present non-trivial features including heterogeneous volume fraction, secondary flows, symmetry breaking and dynamically maintained order. Some of these regimes have been observed experimentally (Holyoake and McElwaine (2012)). Note that the very nature of the incline or of the sidewalls (flat or bumpy) is not crucial to obtain such regimes as long as grain-wall friction prevails over grain-grain friction.

A polydisperse system is more complex to study since it segregates that is, the smallest grains percolate to the bottom, while the largest are squeezed up toward the free surface (Savage and Lun (1988), Weiderseiner et al. (2011)). This process takes time to reach the steady state and it introduces a spatial heterogeneity within the system. The former point requires very long inclines to reach SFD flows or the use of numerical simulation with periodic boundary conditions in the main flow directions. For instance, Tripathi and Khakhar (2011) propose an adaption of the $\mu(I)$ rheology and test it by means of DEM simulations.

Granular/fluid systems in a steady and fully developed state have been investigated through flume experiments (e.g., Armanini et al. (2005), Larcher et al. (2007), Armanini et al. (2009), Kaitna et al. 
(2014)). For example, Armanini et al. (2005) examined particle/fluid mixture in steady uniform conditions by setting up a closed loop with an inclined channel and a high-speed conveyor belt. Such conditions make it possible to analyze the internal structure of the flow using high-speed video imaging through the sidewalls of the flume. These flows show a stratification of rheological regimes (Armanini et al. (2005), Larcher et al. (2007)): an external region, near to the free surface, dominated by nearly instantaneous contacts among the particles (collisional regime), an internal region dominated by prolonged contacts among the particles (frictional regime) and possibly a static bed in which the particles are immobile. Depending on the ratio of the solid to liquid discharges and on the channel inclination, different flow regimes can be sustained: either flows of the mixture over a rigid, nonerodible base (moderate slope and large volume mixture) or flows over an erodible base (larger slopes) (see for further details the review of Berzi et al. (2010) on debris flows). The experimental data drawn from these steady configurations provide invaluable information for testing two phase-flow models.

\subsection{Self-channelized granular flows and levées formation}

Another relevant configuration is truly unconfined flows, i.e. flows whose lateral extension is small compared to the corresponding size of the setup. In practice to obtain such kind of flows, grains are released from a reservoir located at the top of the plane by opening a gate whose width is significantly smaller than that of the incline. Depending on the input flux and on the plane inclination, different flow regimes and associated deposit morphologies are observed when a long lasting supply is imposed upslope of beds with inclination angles close to the friction angles of the granular material involved (Figure 5). Low flux and/or low slope angle lead to intermittent avalanches and rounded deposits, intermediate flux and slope promote self-channelized quasi-steady flows, and high flux and slope cause a roll-wave regime (Félix and Thomas (2004), Takagi et al. (2011)). Note that series of adjacent self-channelized flows form when the gate has the same width as that of the incline, and this phenomenon is called granular fingering (Pouliquen et al. (1997), Pouliquen and Vallance (1999)). In that case small perturbations at the flow front are amplified, which leads to more or less merged granular fingers.

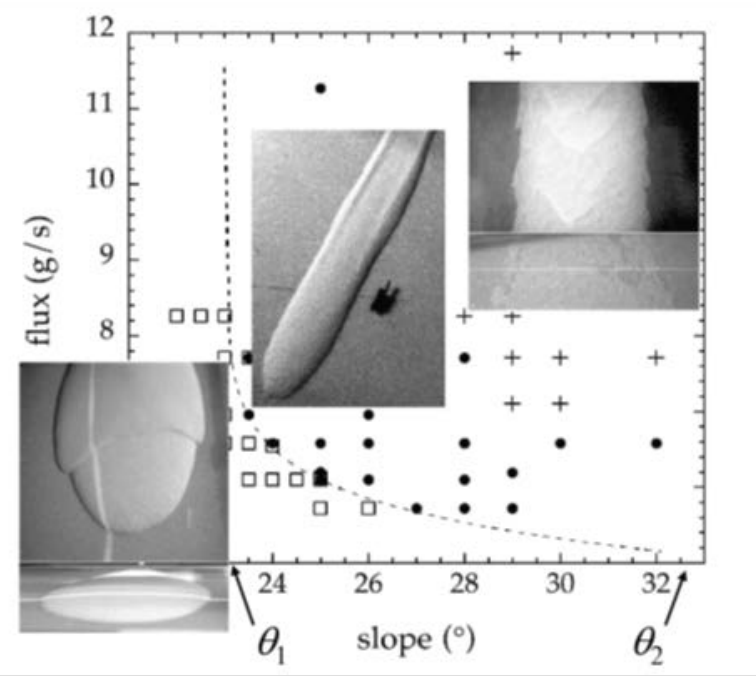

Figure 5. Flow regimes generated by a constant supply of glass beads with narrow size distributions (typically 300-400 $\mu \mathrm{m}$ ) over an inclined bed for different input flux and slope: ( $\square$ ) intermittent avalanches, $(\bullet)$ selfchannelling quasi-steady flow, $(+)$ roll wave regime. The dashed curve represents $h_{\text {stop }}(\theta)$. Figure adapted from Félix and Thomas (2004). Similar flow regimes and deposits are observed for sand particles for higher slopes and fluxes by Takagi et al. (2011).

In the intermediate regime, the flow creates its own channel with an almost constant width bordered by lateral levées along the slope and generates a levées-channel deposit as observed for some pyroclastic flows, snow avalanches, debris or submarine flows (see Figure 2c,d and section 4). The question is as to whether the physical processes at the origin of self-channeling and levées-channel deposit are essentially the same for all these geophysical flows. Important issues are to understand and 
quantify the role of these parameters in the flow dynamics and deposit morphology. For geophysical flows, the challenge is also to find scaling laws relating the deposit morphology to the flow dynamics.

The detailed and comprehensive laboratory experiments of Félix and Thomas (2004) show that self-channeling and levées-channels deposits can be obtained with dry granular flows. They show that self-channeling results from the building up of static lateral zones that develop behind the front during the flow, creating a steady flow in a natural channel of approximately constant width along the slope. The levées-channel morphology appears when the supply of granular material is stopped and the central channel is drained and its thickness decreases. The levées form from the static lateral zones, even though part of these zones collapse during the channel draining (Félix and Thomas (2004), Kokelaar et al. (2014)). When the imposed flux increases, both the total width of the flow (and deposit) $w$ and the front velocity increase, although the velocity seems to saturate at high flux (Takagi et al. (2011)), whereas the thickness of the flow $h_{\mathrm{fl}}$ and of the levées $h_{1}$ only slightly increase and the thickness of the central channel of the deposit $h_{\mathrm{c}}$ remains almost the same. When the slope angle increases, the width $w$ and the front velocity also increase while the morphology is characterized by a smaller thickness of the levées and of the central channel deposit.

Further laboratory experiments and simulations showed that the total width of the lobe $w$ increases slightly with time (Deboeuf et al. (2006); Mangeney et al. (2007a), Takagi et al. (2011)). Deboeuf et al. (2006) assumed that flows, in their experiments with glass beads, reach asymptotically a steady regime, in the long time limit. On the contrary, Takagi et al. (2011) showed that, at long time (after about one hour), glass beads flows become unstable with fluctuations of their width and thickness. On the other hand, long term flows of sand particles reach a steady regime (Takagi et al. (2011)). These contradictory assumptions and observations lead to different interpretation of the flow in terms of rheology (see section IV of Takagi et al. (2011)). This long term behavior, however, does not correspond to the time scale of natural flows that travel and deposit in much shorter time (maximum of $~ 10$ minutes at the field scale corresponding to 10's of seconds at the laboratory scale if the characteristic time scale is taken as $\sqrt{ }(h / g))$.
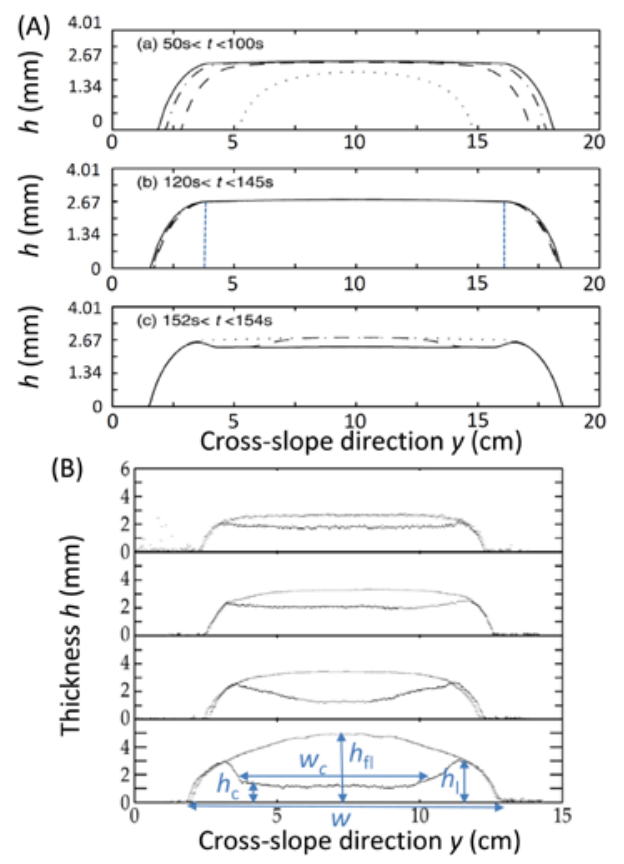

Figure 6: (A) Transverse profiles $h(y) / h_{\text {stop }}$ at a given downslope position along the plane during the flow of the granular lobe (input flux: $12 \mathrm{~g} / \mathrm{s}$, slope:25 $5^{\circ}$ (a) under constant supply at $t=60 \mathrm{~s}$ (dotted line), $t=70 \mathrm{~s}$ (dashed line), $t=80 \mathrm{~s}$ (dashdotted line), and $t=100 \mathrm{~s}$ (solid line); (b) under constant supply at $t=120 \mathrm{~s}$ (dashed line), $t=$ $130 \mathrm{~s}$ (dash-dotted line), and $t=144 \mathrm{~s}$ (solid line), the blue vertical short-dashed lines represent the limit between the inner flow and the quasi-static zones; and (c) during the draining phase at $t=152 \mathrm{~s}$ (dotted line), $t=153 \mathrm{~s}$ (dashed line), and $t=154 \mathrm{~s}$ (solid line). (B) Flow and deposit morphologies for an increasing polydispersity degree, from top to bottom (input flux: $8.15 \mathrm{~g} / \mathrm{s}$, slope: $25^{\circ}$ ). The morphometric parameters of the flow and 
deposit are added in blue on the bottom figure. Figure adapted from Mangeney et al. (2007a) and Félix and Thomas (2004).

Félix and Thomas (2004) showed that the morphologies of the flow and of the deposit are strongly sensitive to the degree of polydispersity (glass beads with different sizes) while the width and velocity of the flowing central channel seem to only slightly depend on it. In particular the levéeschannel morphology is significantly enhanced when the degree of polydispersity increases, i.e. when the size distribution of the grains is wider (Figure 6B). As polydispersity increases, small and large particles concentrate in the channel and the levees, respectively. With colored particles it is possible to evidence the segregation process: large particles migrate to the flow surface while small particles percolate downward through kinematic sieving and accumulate in the basal part of the flow. Because large particles at the flow surface have larger velocity than average, they migrate forward to the front zone where they (i) are directed to the quasi-static borders where they get trapped, or (ii) get entrained to the flow base and then are segregated towards the surface and recirculated (Pouliquen and Vallance (1999), Félix and Thomas (2004)). Based on the segregation model of Gray and Thornton (2005) and on an empirical three-dimensional velocity field resembling the experimental observations of water saturated granular material, Johnson et al. (2012) suggest that the segregation process may be more complicated, involving recirculation of large particles in spiral trajectories within the flow head and that are further advected to the flow edges and deposited to form large-particle-enriched levees. Using angular coarse grains together with more rounded fine particles, as it is frequently the case in nature, Kokelaar et al. (2014) showed that the run-out distance of self-channelized flows strongly depend on polydispersity. In that case, the material enriched in coarse irregular shaped particles within the lateral borders experience higher friction than the fines-enriched more rounded material flowing within the central channel (Pouliquen and Vallance (1999), Takagi et al. (2011), Kokelaar et al. (2014)). Run-out distance is enhanced as the coarse-grained borders prevent lateral spreading and as the channel consists mostly of finer grains with lower friction.

When granular flows are water-saturated, scaling issues are encountered in small-scale laboratory experiments, in particular due to the relatively strong effect of surface tension, pore-fluid shear resistance and cohesion at this scale compared to real situations and to the faster dissipation of excess pore-fluid pressure (Iverson (2015)). The large-scale USGS debris flow facility (95 m long, $2 \mathrm{~m}$ wide, and $1.2 \mathrm{~m}$ deep channel) in Oregon makes it possible to overcome this problem and to mimic real debris flows made of water-saturated mixtures of sand, gravel, and silt or clay. Typical volumes of $\sim 10$ $\mathrm{m}^{3}$ are released from rest on a $31^{\circ}$ inclined channel connected to a $2.4^{\circ}$ unconfined surface (Figure 7). The experimental flows reproduce the architecture of debris flows commonly observed in nature, which consist of a dilated, coarse-grained snout with intense particle collisions and of a nearly liquified, fine-grained body with surface roll waves (Iverson et al. (2010)). When reaching the runout area, self-channelized flows are observed. They are very similar to the self-channelized dry granular flows described above with levées-channel morphology, despite the very different nature of the material involved and the very different slope and input flux (Johnson et al. (2012)).

The presence of a fluid phase, however, may change the segregation processes and the frictional properties of the flow as shown for example by fingering instabilities of propagating granular fronts (Pouliquen and Vallance (1999)). Indeed, the presence of a viscous liquid may decrease the efficiency of segregation processes, which inhibits fingering. On the contrary, size-segregation occurs in granular material fluidized with air or water, thus increasing the permeability of the front and lateral quasistatic borders and reducing it in the inner flow so as to enhance retention of the pore-fluid pressure and significantly increase the flow mobility. This effect is promoted by the presence of fine silt and clay particles in water-saturated mixtures where high pore pressure is maintained up to deposition and even during later consolidation (Iverson (1997), Major and Iverson (1999)). This change in permeability also adds to the size-segregation of irregular coarse particles and more rounded fine particles to increase the difference of friction between the inner low-friction flow and the high friction borders. Therefore, the morphology of the deposit results from both the flow dynamics and the flow composition, making it difficult to relate the deposit characteristics to the flow properties. Félix and Thomas (2004), however, proposed a relation to estimate the flow velocity from the thickness of the levées and of the central channel (see section 4). 
Numerical simulations based on a thin-layer depth-averaged model using the $\mu(I)$ rheology at the leading order makes it possible to reproduce qualitatively most of the laboratory of self-channeling flows without taking into account segregation processes (Mangeney et al. (2007a)). In these configurations, the $\mu(I)$ rheology leads to increase the friction at the border of the flow where the thickness decreases rapidly (Mangeney et al. (2007a)). The simulations reproduce the self-channeling of the flow with quasi-static borders, the slight increase of the total flow width with time, the draining of the central channel when the supply stops, and the resulting levées-channel morphology. The simulated levées-channel deposit looks very similar to the deposit observed in the laboratory for almost monodisperse mixtures of beads (Figure 6). The velocity field is well reproduced with a quasiconstant downslope velocity in the central channel, with velocity decreasing and rotating toward the cross-slope direction when approaching the lateral borders of the granular mass (see e.g. Figure 17 of Mangeney et al. (2007a) and Figure 9 of Johnson et al. (2012)). Analysis of the downslope and crossslope forces shows that the balance between the friction force related to the $\mu(I)$ rheology and the thickness gradients plays a key role in the rotation of velocities near the front, in the occurrence of a quasi-static flow, and in the generation of static zones just behind the front (Figures 13 and 18 of Mangeney et al. (2007a)). By coupling a depth-averaged description of the preferential transport of large particles towards the front (Gray and Kokelaar (2010)) with a classical thin-layer depth-averaged model, Woodhouse et al. (2012) simulated finger formation in a bidisperse granular flow. Numerical simulations reproduce qualitatively the experimentally observed formation of a front rich in large particles, its instability and the subsequent evolution of elongated fingers bounded by large-rich lateral levees. However, the number of fingers increase with the numerical resolution, highlighting the illposedness of the equations in the investigated regime related to the lack of important physical processes in the model. The depth-averaged $\mu(\mathrm{I})$-rheology (Gray and Edwards (2014), Baker et al (2016)) provide a plausible means of regularizing the model and hence determining the development of segregation-induced fingers.

Simulations suggest that, contrary to the total width $w$, the width of the central channel $w_{\mathrm{c}}$ (almost equal to the width of the quasi-steady flow) is almost constant for a given flux and slope. By assuming that the flow thickness is about the same as the levées thickness and that the thickness of the central channel is about equal to $h_{\text {stop }}$, Mangeney et al. (2007a) suggest that the width of the central channel is proportional to the input flux as found in the experiments of Takagi et al. (2011), making it possible to estimate the flux (or the velocity) from the measured heights of the central channel and lateral levées of the deposit.

These experimental and numerical studies suggest that the mechanisms governing selfchannelling and levée formation could be generic to a wide variety of geophysical granular flows, involving or not polydispersity and/or a fluid phase. In other words, grain size segregation and accumulation of coarse particles at flow surface and margins is not necessarily the cause of selfchannelling (though it may contribute to enhance it). Note, however, that grain size segregation seems to be a prerequisite for granular fingering (Pouliquen et al. (1997), Pouliquen and Vallance (1999), Woodhouse et al. (2012)).

However, up to now, simulations of self-channeling flows and levees formation or analysis based on simple models as those described above were compared essentially qualitatively to experimental results. To go further in the understanding and constrain of the physical processes at work, quantitative comparison of simulated and observed thickness and velocity profiles should be performed. Accurate simulation of these processes would require two-phase models describing both the mass and momentum conservation of the solid and fluid constituents and their interaction coupled with segregation models. A key issue is to better describe the behavior at small Froude and inertial numbers corresponding to the regime experienced by the border of the flow (Figure 14 of Mangeney et al. (2007a)). 

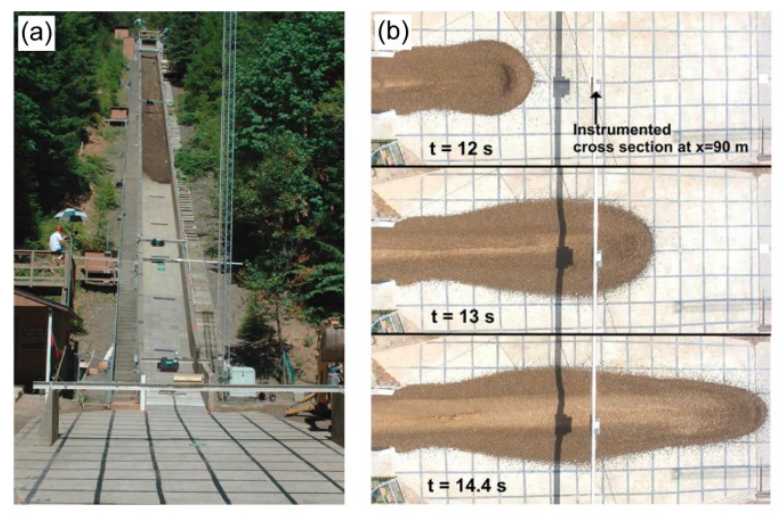

Figure 7: (a) USGS debris-flow large scale facility. (b) Vertical aerial photographs of a debris flow in the runout area, at different times after release. Scale is given by the 1-m grid. In Iverson et al. (2010).

\subsection{Dam-break granular flows}

3.4.1 Insight from laboratory experiments. Unsteady flow generated from the quasi-instantaneous release of a granular column (dam-break configuration, Figure 8) was considered in many experimental studies over the last decade. Such experiments represent simple analogues of geophysical granular flows and can also serve as tests for numerical simulations. Studies involved various types of granular materials with negligible (granular flows) to high internal pore fluid pressure (gas-particle flows and liquid-particle flows), and were conducted in unidirectional (2D) or axisymmetrical (3D) configurations on horizontal or inclined beds. They focused on flow kinematics and on scaling laws relating the morphological characteristics of the flow deposits (run-out, height) to the geometry of the initial column released.

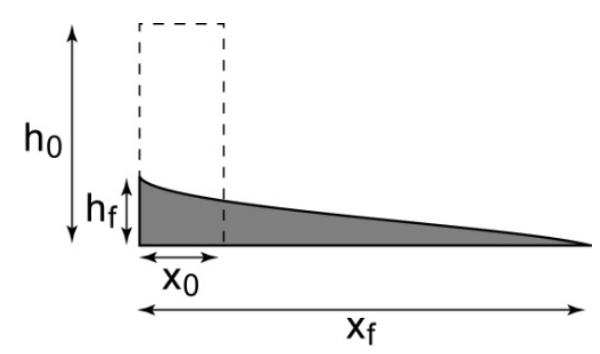

Figure 8. Dam-break granular flow experiments, with released initial column (dashed line) of height $h_{0}$ and length $x_{0}$, and flow deposit of length $x_{\mathrm{f}}$ and height $h_{\mathrm{f}}$.

Experiments show that the flow duration is controlled by a timescale proportional to that of particle free-fall, $t_{\mathrm{f}}=\left(h_{0} / g\right)^{1 / 2}$ where $h_{0}$ is the initial column height. Emplacement occurs in three phases: the flow front accelerates after release, then propagates at nearly constant velocity in some configurations, and finally decelerates until motion ceases (Lajeunesse et al. (2004, 2005), Lube et al. (2004, 2005), Balmforth and Kerswell (2005), Roche et al. (2008)). The run-out distance of dry flows is travelled by material originating from the outer surface of the upper third of the initial column (Thomson and Huppert (2007)). Only part of the granular mass flows while particle sedimentation occurs as the flow propagates, resulting in a basal static region overridden by an upper and frontal part, both being separated by an interface that propagates upwards until the flow is consumed (Lajeunesse et al. (2005), Lube et al. (2007, 2011), Mériaux (2006), Doyle al. (2007), Girolami et al. (2008, 2010), Farin et al. (2014), Ionescu et al. (2015), Lusso et al. (2015a)). Gas-particle flows with high pore pressure notably propagate like inertial fluid gravity currents at velocity $U \sim\left(2 g h_{0}\right)^{1 / 2}$ during most their emplacement, despite their high particle concentration, before they enter a frictional granular regime once pore pressure has decreased sufficiently through diffusion (Roche et al. (2008)).

Experiments on horizontal slope show that the characteristics of the final deposit depend primarily on the column aspect ratio defined as 


$$
R=\frac{h_{0}}{x_{0}}
$$

where $x_{0}$ is the initial column length (see Table 3 and references therein). The deposit shape varies from truncated cone, with slope close to the repose angle of the granular material at low $R$, to cones with slope down to a few degrees at high $R$ (Lajeunesse et al. (2004, 2005), Lube et al. (2004, 2005), Balmforth and Kerswell (2005), Siavoshi and Kudrolli (2005)). The normalized run-out distance

$$
x^{*}=\frac{x_{\mathrm{f}}-x_{0}}{x_{0}}
$$

and deposit height

$$
h^{*}=\frac{h_{\mathrm{f}}}{x_{0}}
$$

where $x_{\mathrm{f}}$ and $h_{\mathrm{f}}$ are the deposit length and height (Figure 8), are used to define scaling laws of the form

$$
x^{*}=\lambda R^{n}, h^{*}=\lambda^{\prime} R^{n^{\prime}}
$$

though a clear law for $h^{*}$ has not been defined yet. Note that $h_{0}$ instead of $x_{0}$ can be used as well to normalize these parameters, so that $x^{*}=\lambda R^{n-1}$ (resp. $h^{*}=\lambda{ }^{\prime} R^{n^{\prime}-1}$ ). Data for $h^{*}$ do not show systematic trends, but those for $x^{*}$ reveal that the value of the coefficient $\lambda$ and of the exponent $n$ depend respectively on the material properties and on the initial configuration (Table 3). Almost all studies show that $n=1$ (i.e. $x^{*}=\lambda R$ ) at low $R$, typically less than $1.6-3$, so that $x_{\mathrm{f}}-x_{0}=\lambda h_{0}$. In other words, the distance travelled by the flow from the edge of the initial column is simply proportional to the initial column height. At higher $R, n<1$ for unidirectional flows but $n$ approaches 1 as the channel width increases and side effects become weaker (Balmforth and Kerswell (2005), Mériaux (2006)). Results of Lacaze et al. (2008) are a notable exception, but their experiments involve flows only one particle wide whose dynamics might differ fundamentally from that of flows that are typically hundreds of particle diameter wide. In the axisymmetrical configuration $n=1 / 2$ at high $R$, which may reflect spreading of the granular mass (Lajeunesse et al. (2004)). Farin et al. (2014) recently showed that these scaling laws are not valid for granular collapse over slopes higher than about one half of the friction angle of the material involved $\left(\theta_{\mathrm{c}}\right)$. In that case, a last regime of slow, almost steady propagation occurs after the initial acceleration and deceleration phases typical of granular collapses over smaller slopes. In that case the normalized quantities vary also with the volume of the released mass.

Other things being equal, parameters causing run-out increase (i. e. higher $\lambda$ ) include the aspect ratio, the slope angle (Hogg (2007), Mangeney et al. (2010), Farin et al. (2014)), the volume of material released and the presence of on erodible granular substrate (Mangeney et al. (2010), Farin et al. (2014)), the material polydispersity with possible related friction reduction effects (Phillips et al. (2006), Roche et al. (2005), Meruane et al. (2012)), and high initial pore fluid pressure (Roche et al. (2011)). For flows within a viscous ambient fluid, low initial particle volume fraction dramatically increases the runout distance because pore pressure is generated due to fluid expulsion as the flowing material contracts. In contrast, initially dense flows with volume fraction close to the maximum packing travel about the same distance as dry flows (Figure 11b of Rondon et al. (2011)). The related mechanisms and their influence on the scaling laws are yet to be investigated in detail; in contrast, runout decrease (lower $\lambda$ ) is caused by both slow material release (i.e. low inertia, Mériaux (2006), increased material friction angles (Balmforth and Kerswell (2005), Roche et al. (2011)) and interparticle cohesion (Mériaux and Triantafillou (2008)). The presence of a thin layer of erodible material lying on an inclined bed may increase the maximum runout distance of a granular avalanche flowing down the slope by up to $40 \%$ and change the flow regimes at slopes higher than the critical angle $\theta_{\text {c }}$ (Mangeney et al. (2010) and Farin et al. (2014)). In that case, as for granular flows on steep 
slopes, a slow, almost steady propagation phase occurs. Note that there could be a variation of a few degrees of this critical angle as a function of the initial and boundary conditions, but this has not been demonstrated yet.

\subsubsection{Insight from analytical and numerical models}

Analytical solution of the thin-layer depth-averaged equations (Mangeney et al. (2010), Faccanoni and Mangeney (2013)) and numerical simulations show that the coefficient $\lambda$ varies inversely with the difference between the tangent of the slope angle and that of the material friction angle (Balmforth and Kerswell (2005), Kerswell (2005), Mangeney-Castelnau et al. (2005), Mangeney et al. (2010)):

$$
\frac{x_{f}}{x_{0}}=\frac{2 k}{\tan \delta-\tan \theta}
$$

with $k$ an empirical parameter ( $k \sim 0.5$ see Mangeney et al. (2010)). This contradicts the simple model proposed by Lajeunesse et al. (2005) where the runout distance is proportional to the friction coefficient $\mu=\tan \delta$. This dependence (3.5) is particularly well illustrated by gas-particle flows with high pore pressure (and hence low internal friction) whose run-out distance is larger (i.e. higher $\lambda$ values) than that of their dry granular counterparts (Roche et al. (2011) and Figure 10B).

While simulations using DEM and depth-averaged thin layer models reproduced the abovementioned experimental scaling laws, very few quantitative comparisons with the deposit extent (i.e. exact value of $\lambda$ ) and the dynamics of the spreading have been performed. Though thin-layer models reproduced the deposit extent with friction angles only a few degrees higher than the typical friction angle of the material involved (Kerswell (2005), Mangeney-Castelnau et al. (2005)) they overestimate the spreading velocity, in particular during the first instants after release. This is notably due to the neglect in thin-layer models of the vertical acceleration, which is particularly important in the beginning of the spreading. On the other hand, 2D DEM (contact dynamics) strongly overestimates the runout distance (up to $40 \%$ ) even with high grain/grain friction (Staron and Hinch (2005, 2007)). To overcome this issue, 3D DEM (molecular dynamics) by Girolami et al. (2012) included a dissipation related to the resistance to rolling to match the experimental results. Lacaze and Kerswell (2009) were able to reproduce quantitatively the runout extent using 3D DEM with a grain/grain friction coefficient of 0.5. Using a set of parameters typical of the $2 \mathrm{D}$ visco-plastic flow law $\mu(I)$ to compare with $2 \mathrm{D}$ DEM, Lagrée et al. (2011) overestimated the runout distance of granular collapse observed experimentally. However, 2D continuum simulation using pressure and/or strain-rate dependent viscoplastic (Ionescu et al. (2015)) or elasto-viscoplastic (Crosta et al. (2009)) flow laws were able to reproduce quantitatively the deposit of granular columns on horizontal and inclined planes, and even the detailed dynamics by taking into account the lateral wall friction (Ionescu et al. (2015), Martin et al. (2015)). These simulations show that a visco-plastic flow law derived from the $\mu(I)$ rheology makes it possible to reproduce quantitatively the transient regimes characterizing granular collapses over horizontal and inclined beds with rheological parameters derived from the experiments, without any fitting procedure. However, it has to be mentioned that for collapses of small aspect ratio typical of real landslides, a constant viscosity (not dependent on pressure and strain rate) defined in the sense of Ionescu et al. (2015) gives results very similar to those obtained with the variable viscosity derived from the $\mu(I)$ rheology (Ionescu et al. (2015)). Note that the initial opening of the gate has a significant effect on the spreading while it does not change the final deposits (Ionescu et al. (2015)), as also observed for solid-fluid mixture dam-break flows (Iverson and George (2014)). This has to be taken into account for quantitative comparison between simulation and experiments.

During granular collapse over rigid or erodible beds, part of the material is flowing over static material. This is qualitatively reproduced by DEM and continuum 2D models (e. g., Lagrée et al. (2011), Ionescu et al. (2015)) as well as by a multilayer shallow models that was able to reproduce for the first time the increase of runout distance of granular flows with increasing thickness of the underlying erodible bed (Fernandez-Nieto et al. (2016)). Analytical solution and numerical simulation of the time change of the static-flowing interface shows that its dynamics is closely related to velocity profiles and to the rheological properties of the material (yield, viscosity) (Lusso et al. (2015a, 2015b)) as discussed in section 2.5. In particular, the penetration of the static-flowing interface within the 
erodible bed is qualitatively reproduced in the continuum 2D models (Lusso et al. (2015a), Crosta et al. (2009), Mangeney et al. (2007b)). Quantitative comparison is missing, however.

\subsection{Waves or wave-like avalanches}

Waves that look similar to surges reported for debris flows (e. g. Iverson (1997), Zanuttigh and Lamberti (2007)) have long been observed in dry granular flows (Savage (1979)) (Figure 9). These individual waves or series of waves have different characteristics, depending on the initial and boundary conditions and on the material involved.

Soliton-like waves are observed for dry granular flows on rigid or erodible slopes with inclination angles close to the friction angles of the material involved (Figure 9). Pouliquen and Forterre (2002) showed that, when a three-dimensional granular cap is released on such an inclined plane covered by a thin erodible bed of thickness $h_{\text {stop }}$ made of the same granular material, a triangular shaped traveling wave that propagates at a constant downslope velocity puts into motion static material from the erodible bed at the front (erosion) and leaves material at the rear (deposition) (Figure 9a). Triangular shaped erosion/deposition waves have also been observed when a perturbation (Daerr (2001), Malloggi et al. (2006) (in air or water)) or a constant flux of material (Börzsönyi et al. (2005, 2008)) are imposed on top of a thin granular layer lying over an inclined plane. The morphology and dynamical properties of these waves or avalanches are qualitatively and quantitatively different for smooth glass beads compared to irregular-shaped granular materials such as sand (Forterre and Pouliquen (2003), Börzsönyi et al. (2005, 2008)) (Figure 9d and e). In particular, Börzsönyi et al. (2008) showed that distinct, well-resolved avalanches made of rough non-spherical grains are bigger and travel faster than those made of spherical glass beads. For inflow conditions close to the minimum depth $h_{\text {stop }}$ required for steady uniform flow to exist, a series of steadily travelling erosion/deposition waves develop (Edwards and Gray (2015)). Forterre and Pouliquen (2003) showed that sand flows are much more unstable than glass beads flows in their setup configuration and that they generate rollwaves (Figure 9f). These waves have smaller amplitudes than erosion/deposition waves, form in slightly deeper flows, and develop at a much larger distance from the perturbation. The most unstable modes (with large amplitude) of roll-waves occur close to the threshold conditions of the flow (low inclinations and thin flows). The generation of large-amplitude waves near the threshold of the flow may have strong implication for natural flows (see section 4).
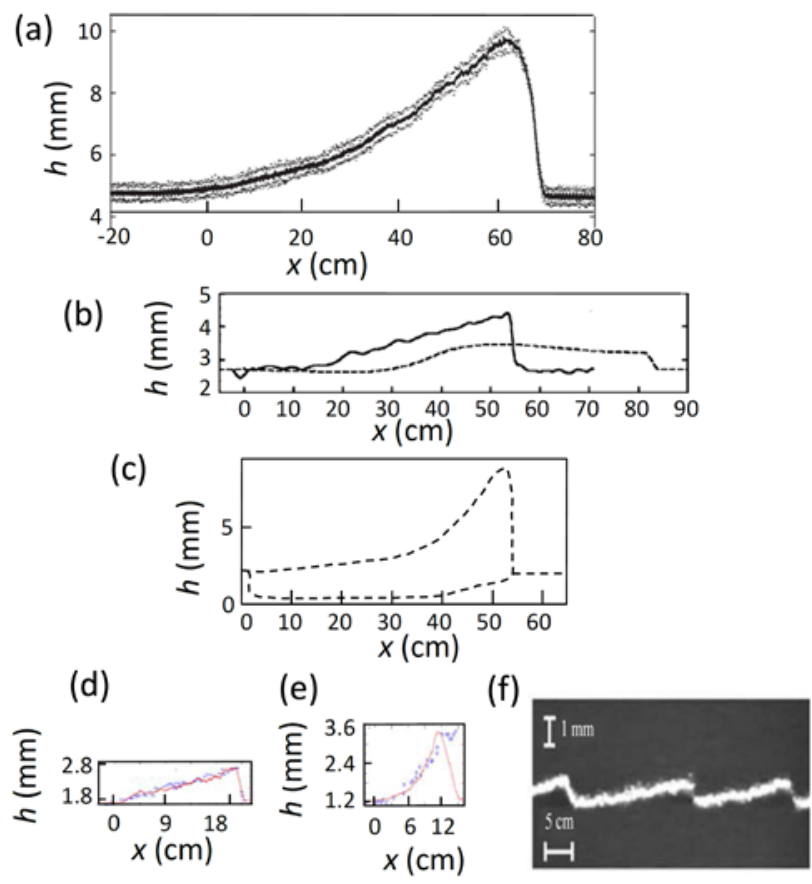

Figure 9. Waves (avalanches) observed for dry granular material flowing on inclined beds with slope angle close to the friction angles of the material involved : (a) Mean and standard deviation of the thickness profile of a sand avalanche generated by an upslope flow rate just below that required for steady flow (diameter $d=0.45 \mathrm{~mm}$, slope 
32 ${ }^{\circ}$ ) (Takagi et al. (2011)); (b) Observed (full line) and simulated with a depth-averaged thin layer model (dashed line) thickness profile of a steady wave created by the release of a granular cap on an initially static layer of glass beads of thickness $h_{\text {stop }}=2.7 \mathrm{~mm}$ (diameter $d=0.5 \mathrm{~mm}$, slope 23०) (Pouliquen and Forterre (2002)); (c) Simulation with the partial fluidization model of the experiment represented in (b) (Mangeney et al. (2007b)), the upper dashed line represents the granular flow free surface and the dahed line below represents the static/flowing interface; (d)-(e) thickness profile (red line) of waves generated by an upslope flux of (d) glass beads ( $d=0.5 \mathrm{~mm}$, slope $\left.24.3^{\circ}, h_{\text {stop }}=1.78 \mathrm{~mm}\right)$ and (e) sand particles $\left(d=0.4 \mathrm{~mm}\right.$, slope $\left.36.8^{\circ}, h_{\text {stop }}=1.2 \mathrm{~mm}\right)$ on an initially static layer made of the same grains and of thickness $h_{\text {stop }}$ (Börzsönyi et al. (2008)); (f) Thickness profile of roll-waves observed on a flow of sand particles $\left(d=0.8 \mathrm{~mm}\right.$, slope $\left.34^{\circ}\right)$ of thickness $h=4.6 \mathrm{~mm}$, generated by an upslope constant flow rate (Forterre and Pouliquen (2003)).

These waves provide a unique test condition for rheological models. Indeed, the precise characteristics of the wave development (amplitude, shape, velocity, stability threshold, growth rate, cutoff frequency, etc.) dramatically depend on the rheological properties of the material. Using the "partial fluidization model” proposed by Aranson and Tsimring (2002) that involves a viscosity proportional to the square root of the pressure and that describes explicitly the static-flowing transition through a phenomenological order parameter, Mangeney et al. (2007b) reproduced qualitatively the steady traveling erosion/deposition wave obtained experimentally by Pouliquen and Forterre (2002). Using the same model, Aranson et al. (2006) identified a family of propagating soliton-like avalanches with shape and velocity controlled by the inclination angle and the thickness of the substrate. At high inclination angles, the solitons display a transverse instability, followed by coarsening and fingering similar to the experimental observation of Malloggi et al. (2006). These simulations, however, only reproduce qualitatively the experimental results (see e. g. Figure 9b-c). Analysis of these waves has also been done with depth-averaged thin-layer models, where the whole layer of grains at a given downslope position is implicitly assumed to be either completely mobile or completely static. Pouliquen and Forterre (2002) showed that depth-averaged thin layer models with the $\mu(I)$ rheology implemented at leading order does not reproduce the erosion wave they observed experimentally (Figure 9b). Using the same model, Forterre and Pouliquen (2003) showed that roll waves observed for glass beads and for sand particles result from the same instability mechanism and that their different behaviour could be explained by the quantitative differences in the friction coefficient of the $\mu(I)$ rheology. This model, however, is unable to predict the observed cutoff frequency. The cutoff frequency results from the dissipation due to the longitudinal velocity gradients (the extensional viscosity term) that are neglected in leading order thin-layer models (Forterre (2006)). By adding a depth-averaged viscous term (Gray \& Edwards 2014), based on the $\mu(\mathrm{I})$-rheology, into the model, Edwards \& Gray (2015) showed that it was possible to predict the formation of erosion-deposition waves similar to those shown in figure 9, which have completely static zones between steadily traveling mobile regions. In particular, this theory was able to accurately capture the growth, coarsening dynamics and typical wave amplitudes and lengths, which is an important step forward in the goal to incorporate erosion and deposition into depth-averaged theories. Börzsönyi et al. (2005, 2008) use also depth-averaged thin layer models with the leading order $\mu(I)$ rheology to explain the difference between the avalanches generated by glass beads or sand, no quantitative comparison with the avalanche shape and velocity was achieved.

Waves at the interface between a flowing granular mass and an initially static erodible bed were also observed experimentally (Mangeney et al. (2010), Farin et al. (2014)). They could have tremendous effect on the amount of bed material entrained (Rowley (2011)). Modelling these waves could be a unique way to investigate rheological laws near the static-flowing transition. Furthermore, a challenging issue is to quantify the role of interstitial fluid and grain polydispersity on erosion/deposition and roll waves development to ultimately compare them with natural surges (e. g. Iverson and Denlinger (2001), Zanuttigh and Lamberti (2007), Zanuttigh and Ghilardi (2010)). Indeed, natural surges are strongly heterogeneous as they are generally composed of a first surge, mainly containing boulders, and of possible secondary waves that are generally muddier as shown in large scale experiments (see section 3.3 and Figure 7).

\subsection{Interaction of granular flows with complex topographies}

Experiments of granular flows over complex topographies provide useful tests for numerical models (see Pudasaini et al. (2007) for a review). Simulations of these experiments attempted to investigate 
the anisotropy of normal stresses in granular flows. Considering this anisotropy, however, did not permit reproducing quantitatively the experiments (Pirulli et al. (2007)), while adding different coefficient for along-slope and transverse normal stress anisotropy seemed to improve the results (McDougall and Hungr (2004)). Quantitative agreement with the experiments instead required to add an ad-hoc variation of the friction angle towards the tail of the flow (Gray et al. (1999)). In similar experiments by Iverson et al. (2004), avalanches of dry sand were confined within a sloping irregular 'valley' before they stopped on a horizontal plane. Using a depth-averaged thin layer model that took into account anisotropy of normal stresses and an approximation of the vertical acceleration that compensates the thin-layer approximation made in the horizontal/vertical reference frame (see section 2.6), they were able to simulate some characteristics of the flows, with friction coefficients measured independently with a tilt table apparatus. Details of the simulated dynamics and deposit were however quite different from those in the experiments (Iverson et al. (2004), their Figures 9, 10). Using a similar model that took into account all the curvature terms of the topography derived from the thinlayer approximation applied in a reference frame tangent to the topography, Lucas et al. (2014) (see their Figure 6 in the supplementary material) also reproduced these experiments but without including anisotropy of normal stresses. As a result of the complex interplay between topography and rheological effects and because of the difficulty of properly taking into account topography in depthaveraged thin-layer models, more detailed comparison of the simulated and observed dynamics and deposit should be performed to be able to really discriminate relevant rheological laws.

Granular flows around obstacles (Tai et al. (2001), Gray et al. (2003), Hákonardóttir and Hogg (2005), Vreman et al. (2007), Gray and Cui (2007), Faug et al. (2008), Benito et al. (2012)) are also of paramount importance given the applications to defensive structures (e.g. deflecting and catching dams) aiming to protect people and infrastructure from hazardous natural granular flows. As an example, Benito et al. (2012) showed that presence of a forest of cylinder obstacles significantly increase the stability of a granular layer. Moreover, such kind of flows is also interesting from a fundamental point of view. Indeed they generate shock waves at which there are rapid changes in the flow properties (thickness, velocity etc.). Yet, they can be used as benchmarks to test theories, models or numerical simulations aiming to describe granular flows. It has been recently shown that a thinlayer depth-averaged model assuming isotropy of normal stresses and a constant friction coefficient (Cui and Gray (2013)) captures experimental results in a satisfactory way. A precise description of such flows is out of the scope of the paper and the interested reader should refer to the aforementioned references for more details.

Table 3. Scaling laws in experiments on horizontal dam-break granular flows.

\begin{tabular}{lll}
\hline $\begin{array}{l}\text { Configuration } \\
\text { Reference }\end{array}$ & $x^{*}=\left(x_{f}-x_{0}\right) / x_{0}$ & $h^{*}=h_{f} / x_{0}$ \\
\hline
\end{tabular}

\section{Unidirectional}

Balmforth and Kerswell (2005)

Lajeunesse et al. (2005)

Lube et al. (2005)

Lacaze et al. (2008) ${ }^{\text {a }}$

Mériaux (2006) ${ }^{\mathrm{b}}$ $\propto R^{0.55 \pm 0.05}(w=1 \mathrm{~cm}, R>2)$

$\propto R^{0.9 \pm 0.1}(w=20 \mathrm{~cm}, R>2)$

$\propto R(R<3)$

$\propto R^{2 / 3}(R>3)$

$1.6 R(R<1.8)$

$2.2 R^{2 / 3}(R>2.7)$

$1.2 R(R<1.8$, symmetric $)$

$1.9 R^{2 / 3}(R>2.7$, symmetric)

$\propto R(R<2.2)$
$\propto R^{0.72 \pm 0.03}(d=2.5 \mathrm{~mm}, R>2.7)$
$\propto R^{0.83 \pm 0.03}(d=5 \mathrm{~mm}, R>2.7)$

$0.8 R$ (sand, $R<2$ )

$R$ (glass beads, $R<2$ ) $\propto R^{0.45 \pm 0.05}(R>1.5)$

$\propto R(R<0.7)$

$\propto R^{1 / 3}(R>0.7)$

$\propto R^{2 / 5}(R>1.15)$

$\propto R^{0.45 \pm 0.02}(d=2.5 \mathrm{~mm}, R>1)$

$\propto R^{0.39 \pm 0.02}(d=5 \mathrm{~mm}, R>1)$

$R(R<2)^{\mathrm{C}}$

$1.1 R^{0.45 \pm 0.05}$ (sand, $R>2$ ) 


$$
\begin{aligned}
& \propto R^{0.70 \pm 0.05} \text { (sand, } R>2 \text { ) } \\
& \propto 1.3 R^{0.70 \pm 0.05} \text { (glass beads, } \\
& R>2 \text { ) }
\end{aligned}
$$

\section{Axisymmetric}

Lajeunesse et al. (2004)

Lube et al. (2004)

Roche et al. (2011)

$$
\propto R(R<3)
$$$$
\propto R^{1 / 2}(R>3)
$$

$$
1.24 R(R<1.7)
$$$$
1.6 R^{1 / 2}(R>1.7)
$$

$$
\begin{aligned}
d= & 330 \mu \mathrm{m} \\
& 1.40 R(R<1.6) \\
& 1.82 R^{1 / 2}(R>1.6) \\
& 1.48 R(\text { fluidized, } R<1.6) \\
& \left.1.99 R^{1 / 2} \text { (fluidized, } R>1.6\right) \\
d= & 80 \mu \mathrm{m} \\
& 1.61 R(R<1.6) \\
& 1.84 R^{2 / 3}(R>1.6) \\
& 2.49 R(\text { fluidized, } R<1.1) \\
& \left.2.53 R^{1 / 2} \text { (fluidized, } R>1.1\right)
\end{aligned}
$$

$R^{0.45 \pm 0.05}$ (glass beads, $R>2$ )

$R(R<0.74)$

$0.74(R>0.74)$

$$
\begin{aligned}
& R(R<1) \\
& 0.88 R^{1 / 6}(1.7<R<10) \\
& \propto R^{-y}(R>10)^{\mathrm{d}} \\
& d=330 \mu \mathrm{m} \\
& \quad R(R<0.7) \\
& \quad 0.7(0.7<R<7) \\
& \propto R^{-1 / 2}(R>7) \\
& 0.26 R^{1 / 3}(\text { fluidized, } R<5) \\
& R^{-1 / 2}(\text { fluidized, } R>5) \\
& d=80 \mu \mathrm{m} \\
& \quad \propto R(R<1) \\
& \sim 0.95(1<R<6) \\
& \propto R^{-1 / 2}(R>6) \\
& \sim 0.1-0.2 \text { (fluidized) }
\end{aligned}
$$

Experiments involved coarse particles of typical grain size $d>\sim 0.3 \mathrm{~mm}$, unless specified. In unidirectional experiments, $w$ is the width of the channel. ${ }^{\mathrm{a}} w=1.2 d$, ${ }^{\mathrm{b}}$ slow gate withdrawal, ${ }^{\mathrm{c}}$ the exponent $n$ is unknown, ${ }^{\mathrm{d}}$ recalculated from $h_{0} / h_{\mathrm{f}}$.

\section{Reconciling laboratory and field observations}

\subsection{Geophysical flows: field measurements}

Field observations provide important constraints on the initial and final conditions (volume and nature of the material involved, type and duration of the mass release, triggering process, deposit morphology...), on the material properties, on the boundary conditions (nature of the ambient fluid, underlying topography, type of substrate...), and on the mechanisms that control the dynamics and deposition of geophysical flows.

Field data mostly consist of measurements of the morphology and extent of the deposits, provided surface erosion was not significant since the time of deposition (Table 4). Very high resolution Digital Elevation Models (DEM), i. e. topography data, with precision down to 1 meter are obtained through the increasing precision of satellite imagery on Earth and on the planet Mars (e.g. Huggel et al. (2005), Lucas et al. (2011, 2014)). In fact, free satellite images of the surface of Mars that has a small erosion rate and no vegetation provide unique data on landslide deposits that are hardly obtained on Earth (Quantin et al. (2004), Mangold et al. (2010), Lucas et al. (2011, 2014), Jouannic et al. (2012)). In subaerial environment on Earth, the shape of the front deposit as well as lateral levées may be measured using terrestrial or airborn scanner laser measurements (LiDAR) reaching a precision of about $2 \mathrm{~cm}$ at about $1 \mathrm{~km}$ of distance (Sovilla et al. (2010), Conway et al. (2010), Jessop et al. (2012), Jaboyedoff et al. (2012)). In submarine environment, bathymetry data with a resolution down to $50 \mathrm{~cm}$ have been obtained locally with a multi-beam echo sounder mounted on a Remotely Operated Vehicle (ROV) and have revealed detailed morphological features (Figure 2d and Cannat et al. (2013)). Sidescan sonar is well suited to identify subaqueous morphological features of deposits of turbidity currents (Piper et al. (1985)). On planetary surfaces, thermal infrared imagery is used to discriminate landslide deposits from the surrounding substrate, especially at night time where the temperature largely depends on the thermal inertia of the surface that is mainly related to grain size and degree of induration. The volume of material released is determined readily if the deposit is well preserved and 
if the underlying topography can be constrained accurately. In cases of severe erosion and/or the topography is unknown, the volume can be inferred from the geometry of the source zone from where the flow initiated. Accurate determination of this geometry, however, is commonly challenging because of multiple collapse events (e.g. Lucas et al. (2011)). Successive topography or bathymetry measurements before and after specific events makes it possible to better constrain the volume involved and to precisely measure the amount and spatial distribution of erosion and deposition (Conway et al. (2010), Le Friant et al. (2010)).

Direct measurement of the flow properties during propagation, however, is very difficult because of the unpredictability and destructive power of the natural phenomena. Yet, parameters such as flow velocity and basal stresses have been quantified from direct measurements (e. g. Arattano and Marchi (2008), McCoy et al. (2013)) or inversion of seismic data (e. g. Brodsky et al. (2003), Favreau et al. (2010)) as discussed in the following. Subaerial flows are obviously more investigated than their subaquous equivalent. Debris flows and snow avalanches are the most documented events (e.g. Marchi et al. (2002), McArdell et al. (2007), Vriend et al. (2013)). However, the number of studies on rockfalls, rock avalanches and pyroclastic density currents involving acoustic or seismic signals is increasing (e. g. Deparis et al. (2007), Ripepe et al. (2009), Hibert et al. (2011, 2014a, 2014b), Levy et al. (2015), Farin et al. (2015)). National observatories record series of complementary data (video, seismic, acoustic) on gravitational flows coupled with pluviometry, ground deformation, and soil properties measurements that are made freely available (e. g. the Observatoire Volcanologique du Piton de la Fournaise (OVPF) where more than 20 years of data are available, or the Observatoire Muldisciplinaire des Instabilités de Versants (OMIV)). Table 4 summarizes the parameters that can be measured in the field and the techniques used for such measurements. Examples of field measurement of parameters are presented below, and the principles and main limitations of the methods employed are highlighted.

Table 4. Parameters of geophysical flows and their deposits, and methods used for measurement. Modified from Arattano and Marchi (2008).

\begin{tabular}{|c|c|}
\hline Parameter & Method \\
\hline \multicolumn{2}{|l|}{ Flow } \\
\hline $\begin{array}{l}\text { Mean }^{(a)} \text { and surface } \\
\text { velocity and flow duration }\end{array}$ & $\begin{array}{l}\text { (a) Ultrasonic sensors, geophones, pressure sensors, infrasound } \\
\text { sensors, seismometers ; }{ }^{(b)} \text { Doppler speedometers, video recordings }\end{array}$ \\
\hline Flow depth & Radar sensors, wire sensors, ultrasonic sensors, laser \\
\hline $\begin{array}{l}\text { Granulometry, particle } \\
\text { concentration }\end{array}$ & Direct sampling \\
\hline Substrate erosion & Scour sensors, buried radars \\
\hline $\begin{array}{l}\text { Basal (normal or shear) and } \\
\text { impact force }\end{array}$ & Load cells, piezoelectric sensors, seismometers \\
\hline Pore fluid pressure & Pressure sensors \\
\hline $\begin{array}{l}\text { Ground vibration } \\
\text { sound }^{(\mathrm{d})} \text { and }\end{array}$ & $\begin{array}{l}\text { (c) Seismometers, geophones (velocimeters, accelerometers) ; }{ }^{\text {(d) }} \\
\text { microphones }\end{array}$ \\
\hline Particle collisions & Geophone \\
\hline \multicolumn{2}{|l|}{ Deposit } \\
\hline Granulometry, particle density & Sampling \\
\hline $\begin{array}{l}\text { Area, volume, flow runout, } \\
\text { morphology }\end{array}$ & $\begin{array}{l}\text { Aerial and satellite images, stereo-photogrammetry, GPS, theodolite, } \\
\text { LiDAR, sidescan sonar }\end{array}$ \\
\hline
\end{tabular}

${ }^{\mathrm{a}-\mathrm{d}}$ Corresponding parameters and methods.

The flow velocity is measured by various means. A direct approach to determine the flow surface and front velocity consists of analysis of videos (Turnbull and McElwaine (2007), Doyle et al. (2011)). 
However, dense basal pyroclastic flows and snow avalanches, for instance, are often obscured by an upper dilute ash or powder cloud, respectively, which prevents measurement of the underlying dense flow velocity. Doppler speedometers are used to measure the surface velocity whereas the mean front velocity can be calculated from pairs of ultrasonic sensors or geophones at a known distance along the pathway, as done for instance for debris flows (see Arattano and Marchi (2008) and references therein). The velocity of snow avalanches can be deduced from pressure data obtained with pitot-type devices (Nishimura and Ito (1997)). In that case, however, a correction must be made according to the size of the piezometer tube. New high resolution radar measurements permit to penetrate through the powder cloud and measure the velocity of the dense snow avalanche front over its entire path with spatial resolution smaller than $1 \mathrm{~m}$ (Vriend et al. (2013)). Correlation of the signals from an array of infrasound sensors or of seismometers makes it possible to localize the source of radiated energy as a function of time and to deduce the flow velocity of pyroclastic density currents, snow avalanches, and rockfalls or individual blocks (Vilajosana et al. (2007), Ripepe et al. (2009), Lacroix et al. (2012)). As strong winds may generate noise when using infrasound sensors, these have to be buried and equipped with pipes connected to the atmosphere (Ripepe et al. (2009)). Flow duration can be deduced from seismic data. Indeed, combined photogrammetric and local seismic data (source-station distances of $\sim 1 \mathrm{~km}$ ) have shown that the flow duration of rockfalls of volume of $10^{1}-10^{4} \mathrm{~m}^{3}$ almost corresponds to the duration of the generated seismic signal (Hibert et al. (2011, 2014a). Similar observations have been made at larger scale (source-station distances of 30 to $300 \mathrm{~km})$ for bigger events $\left(1-5010^{6} \mathrm{~m}^{3}\right.$, Favreau et al. (2010)). Various techniques have been elaborated to measure the flow depth. These include particularly radar and wire sensors (Arattano and Marchi (2008)) as well as laser devices for debris flows generated in large-scale experimental facility (Major and Iverson (1999), Iverson et al. (2010)).

Basal forces, related to normal and shear stresses as well as particle impacts, are measured with load cells or piezoelectric sensors in debris flows (Major and Iverson (1999), McArdell et al. (2007), Iverson et al. (2010), McCoy et al. (2013)) or in snow avalanches (Nishimura and Ito (1997), McElwaine and Turnbull (2005)), respectively. The basal normal stress permits to infer the flow depth or the bulk density if the other parameter is known, whereas the ratio of the normal to the shear stress gives the Coulomb basal friction while the impact force indicates the flow speed if a flow density is assumed. Note, however, that inertial forces related to topography curvature, if present, can significantly affect the basal stresses making it more difficult to relate them to the flow depth or bulk density (Favreau et al. (2010), Moretti et al. (2015)). The interstitial fluid has a fundamental influence on the dynamics of many geophysical flows. For instance pore pressure measurements reveal a stagnation pressure in front of snow avalanches while the flow head generates a relative underpressure, less than that of the ambient atmosphere (Nishimura et al. (1995), McElwaine and Turnbull (2005)). Basal excess pore fluid pressure measured with appropriate sensors indicates either the amount of the weight of the particles supported in flows close to maximum packing or the particle concentration in more dilute mixtures. Excess pore pressure generated by soil contraction (Iverson et al. (2000)) and/or heavy rain falls (Montgomery et al. (2009)) can be responsible of flow initiation as frictional forces are severely reduced. High pore pressure is also measured during emplacement of debris flows, hence providing an explanation for their high mobility (Major and Iverson (1999), McArdell et al. (2007), Iverson et al. (2010), Doyle et al. (2011)). The temporal evolution of the basal forces can also be recovered from seismic data. Indeed, as subaerial or submarine geophysical flows travel down slope they apply stresses to the ground, which generate seismic waves that can be recorded by regional seismic networks and even global networks for very big events (e. g., Kanamori and Given (1982), Kawakatsu (1989), La Rocca et al. (2004), Brodsky et al. (2003), Favreau et al. (2010), Lin et al. (2010)). As an example, the $50 \times 10^{6} \mathrm{~m}^{3}$ Mount Steller landslide, Alaska, generated a vertical ground velocity of about $10^{-4} \mathrm{~m} / \mathrm{s}$ at $37 \mathrm{~km}$ from the source and was recorded by stations located more than $1500 \mathrm{~km}$ away (Moretti et al. (2012), Zhao et al. (2015)). The low frequency (0.01-0.05 Hz) basal force can be calculated by inversion of seismic data using the Green's function of the Earth between the source and the seismometer (e. g. Moretti et al. (2012, 2015), Yamada et al. (2012, 2015), Allstadt (2013), Ekström and Stark (2013)). As an example, the maximum force associated with the MountSteller landslide is $2 \times 10^{11} \mathrm{~N}$. Note that broadband seismometers are required for source inversion because short-period instruments only measure frequencies higher than $1 \mathrm{~Hz}$. 
When a sufficient number of events can be recorded at a same site, the ratio between the loss of potential energy and the high frequency $(>1 \mathrm{~Hz})$ radiated seismic energy can be estimated (Hibert, et al. (2011), Levy et al. (2015)). This energy ratio typically ranges in between $10^{-3}$ and $10^{-5}$, in agreement with experimental results of acoustic emission generated by block impacts (Farin (2015), Farin et al. (2015, 2016)). As a result, estimates of the rockfall volume can be calculated from the radiated seismic energy using simple formula derived from granular flow or impact modeling (Hibert et al. (2011) for granular flows and Farin et al. (2015) for rock impacts). For a given site, purely empirical relations can be also derived from rockfalls seismic signals and independent measurements of the volume involved (Deparis et al. (2007), Dammaeir et al. (2011)). The parameters of these relations depend on the material involved and on the local topography and ground structure. Attempts have also been made to relate the seismic signal frequency to interparticle and flow-substrate interactions. The frequency of the seismic signals associated to debris flows is assumed to reflect their solid concentration so that sliding frictional dense flows should produce lower frequency signals than more dilute flows with more collisions (see Doyle et al. (2011) and references therein). For debris flows, McArdell et al. (2007) have inferred the number of impacts of particles with a diameter typically larger than a few centimetres. This method, however, is very difficult to apply to dense dry granular flows as suggested by the experiments of Farin (2015).

Other parameters can be measured in the field. Substrate erosion by the flows is investigated using buried radars (Sovilla et al. (2006, 2010)), scour sensors consisting of an array of erodible elements (Berger et al. (2011)) or stress and pore fluid pressure sensors (McCoy et al. (2012)), whereas sampling in debris flows and powder snow avalanches is a direct way to know the local particle concentration and/or the grain size distribution (Doyle et al. (2011), Rastello et al. (2011)). Scanner laser measurements may also provide the size and shape of individual grains as well as rugosity of the surface deposit (Haas et al. (2012)). Grain size, particle density and morphology, componentry and textural analysis of the deposits in aerial environment allow to investigate flow physical processes such as particle segregation, fragmentation and abrasion, as well as substrate erosion and melting due to friction (e.g. Calder et al. (2000), Goren et al. (2010), Imre et al. (2010), Manga et al. (2011)). Petrographic analysis at both macroscopic and microscopic (e.g. X-ray diffractometry, Mössbauer spectroscopy on thin sections) scales makes it possible to quantify the conditions and characteristics of frictional melting of the material involved (Weidinger et al. (2014)).

Further field measurements are required to better understand, for instance, the rate of entrainment of the surrounding fluid, the rate of mass exchange between a dense underflow and its upper dilute parts, the amount of erosion of the substrate, and the onset of flow deposition. Substrate erosion is particularly critical in controlling the flow runout distance, as shown by recent experimental studies on either dry or water-saturated flows (Mangeney et al. (2010), Iverson et al. (2011), Farin et al. (2014)), and this issue should become the focus of more studies in the next future. Measurement of particle fragmentation in natural flows is also an open and key issue. Beyond the measurements of the properties of individual landslides, seismic data provide a unique tool to detect, localize and characterize gravitational flows. This provides a new way to quantify and understand the potential link between gravitational activity and external forcing. As an example, recent analysis of rockfall events together with volcanic seismicity at Piton de la Fournaise (La Réunion island) suggests that the volume of rockfalls increases just before magma intrusions reach the surface, hence providing an indicator for eruptions (Hibert et al. (2014a)). Satellite imagery of series of landslides provide complementary data that makes it possible, for example, to quantify links between gravitational instabilities and large earthquakes (Meunier et al. (2007)).

\subsection{From laboratory modelling to field observations}

Before illustrating how laboratory experiments can be used to interpret field observation, let us recall the fundamental issues related to this approach. First, landslide dynamics and deposits are strongly affected by the natural topography, which is generally complex and variable from site to site. On the contrary, laboratory experiments are generally performed in simple configurations. As a result, quantitative interpretation of natural flows in terms of physical processes and rheological behaviour based on laboratory experiments is commonly very difficult. One way to make the link between laboratory experiments and field data is to perform numerical models. The aim is to design models that reproduce quantitatively laboratory experiments and then to apply these models to natural flows 
by taking into account the real local topography. This approach is illustrated in section 4.2.1 below through the challenging question of the high mobility of large landslides. However, both laboratory and numerical models always represent a huge simplification of natural processes. Indeed, natural flows result from a complex interplay between the effects of external forcing, heterogeneity and polydispersity of the material involved, presence of fluids, initial and boundary conditions, nature and entrainment of the substrate, etc. Even when the physical laws describing these processes may be estimated, real field conditions defining the parameters involved in these laws are rarely known and may also change with time during one landslide (e. g. fluid content or grain size distribution of the particles). As an example of the natural complexity, during landslide initiation stage, the mass generally consists of a cohesive rock that destabilizes due to different processes such as the increase of pore pressure due to rain falls or changes in the surrounding stresses due to seismic or volcanic events or groundwater table variations, erosion processes, aging, etc. (e. g. Chigira et al. (2003), Dunning et al. (2007), Helmstetter and Garambois (2010), Kean et al. (2013), Cappa et al. (2014)). Once destabilized the mass gets progressively fragmented in a way that is essentially not understood nor modelled experimentally or numerically (e. g. De Blasio and Crosta (2014)). Finally, the respective contribution of the physical processes (e. g. pore-fluid viscosity, capillarity forces, etc.) may be very different at small and large scale (e. g. Iverson (2015)). The use of appropriate parameters for each scale makes it however possible for the same model to describe both laboratory and field flows (i. e. the terms in the equations will be the same but their relative magnitude will be different).

Despite this natural complexity, 'simple' laboratory experiments can be used to test if precise field observation (deposit extent, morphological features such as levees or fronts) and associated scaling laws may be qualitatively or quantitatively reproduced with or without specific physical processes (e. g. presence of fluid, particle segregation). Combining these experiments with analytical and numerical models makes it possible to understand the origin of these field data and scaling laws and to relate the parameters involved to the properties of the flowing material. Experimental and numerical models may also highlight specific behaviours, suggesting to look for specific features in the field or to revisit field data interpretation as illustrated in the following.

4.2.1 Effective friction and mobility of small to large landslides. The initial aspect ratio of the granular mass released to create natural landslides is generally smaller or of the order of one $(R \leq 1)$ (e. g. Legros (2002), Lucas et al. (2014)). Experimental dam-break experiments and their analytical and numerical modelling described in section 3.4 show that the scaling law $x^{*}=\left(x_{\mathrm{f}}-x_{0}\right) / x_{0}=\lambda R$ is observed on horizontal to moderate slopes (smaller than about half the repose angle of the material involved) and that the parameter $\lambda$ could be approximated by $\mathrm{k} /(\tan \delta$ - $\tan \theta)$, where $\delta$ is the effective friction angle, $\theta$ the slope angle and $k$ a constant (equation (3.5)). For higher slopes, this parameter depends on the volume involved (Farin et al. (2014)). This scaling law is recovered by numerical thinlayer depth-averaged models for different shapes of the initial released mass (Figure 10C and Lucas et al. (2011)). The effective friction experienced by the grains $\mu_{\text {eff }}=\tan \delta_{\text {eff }}$ can be deduced from (3.5) as a function of the slope and geometrical characteristics of the initial mass and deposit:

$$
\mu_{\text {eff }}=\tan \theta+\frac{h_{0}}{x_{\mathrm{f}}-x_{0}}
$$

As shown in Figure 10B, $\lambda$ increases for flows with interstitial pore fluid pressure (obtained by fluidizing fine particles in laboratory experiments), reflecting the lower effective friction experienced by the grains (Roche et al. (2011)). Using (4.1) gives values of $\delta_{\text {eff }}=25^{\circ}-28^{\circ}$ for flows of coarse particles that cannot retain pore pressure, which is of the order of the repose angle of the particles involved, and $\delta_{\text {eff }}=14^{\circ}-17^{\circ}$ for flows of fine particles in which pore pressure decreases slowly (Figure 10B and Roche et al. (2011)).

When trying to recover this scaling law for a series of about 40 well constrained landslides of different sizes in various environments and flowing over different slopes on Earth and on other planets, Lucas et al. (2014) show that there is a very large dispersion of the data (Figure 10A). Using (4.1) with the real values of $h_{0}, x_{\mathrm{f}}, x_{0}$ and $\theta$ for each landslide leads to a wide range of values for $\mu_{\text {eff }}$. This may explain the dispersion of the data shown in Figure 10A as detailed below. On the contrary, when cxonsidering only very large landslides that occurred in the same environment (Valles Marineris 
on Mars) over gentle slopes of about $2^{\circ}$, the data obey to the scaling law given by (3.4) (Figure 10B). The difference between the two data sets of Valles Marineris landslides represented in Figure 10B highlights the difficulty of obtaining field measurements. Indeed, the data are different because Lucas et al. (2014) only considered landslides that were not stopped by the opposite valley wall and used more precise satellite data than those available up to 2006. Furthermore, each point in Lucas et al. (2014) represents a landslide while the points of Lajeunesse et al. (2006) result from the averaging of several landslides. However, whatever the data set, the coefficient $\lambda$ of the scaling law is much smaller than that of lab-scale dry granular flows. This suggests a much higher mobility of large Martian landslides than experimental granular flows in contrary to the conclusion of Lajeunesse et al. (2006). Simulating these landslides based on precise Digital Elevation Model deduced from satellite data with a thin-layer depth-averaged model with only one free parameter, the effective friction coefficient, leads to very small friction coefficients between 0.1 and 0.16 (friction angles of about $6^{\circ} \leq \delta \leq 9^{\circ}$ ) (Lucas et al. (2014), Table 1 of the Supplementary), which are similar to those in experiments on granular flows with reduced friction caused by pore fluid pressure (Roche et al. 2011). Interestingly, the friction angles making it possible to reproduce numerically the extension of these Martian landslides occurring in the same environment and with a similar range of volumes $\left(10^{10} \mathrm{~m}^{3} \leq V \leq 2.5\right.$ $10^{12} \mathrm{~m}^{3}$ ) are almost the same, i. e. varying by less than $3^{\circ}$ (Lucas and Mangeney (2007), Lucas et al. (2011)).
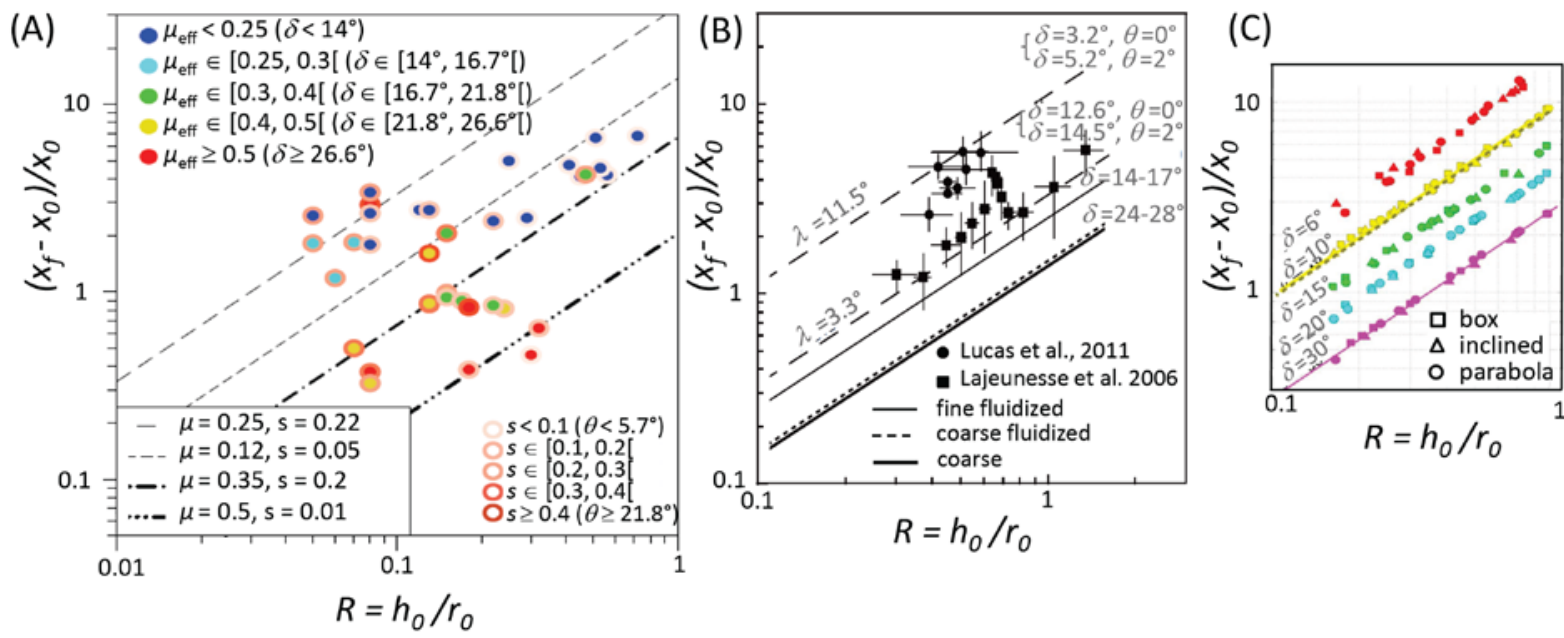

Figure 10: Normalized runout $x^{*}=\left(x_{\mathrm{f}}-x_{0}\right) / x_{0}$ as a function of the initial aspect ratio $R=h_{0} / x_{0}$. (A) Data from real landslides obtained by field observation and satellite imagery (adapted from Lucas et al. (2014)). Each circle represents a landslide where the colour inside the circle scales with $\mu_{\text {eff }}$ calculated with (4.1) while the colour of its contour scales with the mean slope $s=\tan \theta$. The plain lines represent the theoretical curves calculated with (4.1) with some chosen values of $\mu=\tan \delta$ and $s=\tan \theta$. Error bars are approximately twice the size of each symbol. (B) Data from Valles Marineris landslides on Mars (Lucas et al. (2011) and Lajeunesse et al. (2006)) and from laboratory experiments of Roche et al. (2011) for dry and initially fluidized coarse and fine particles (modified form Roche et al. (2011)). The fiction and slope angles correspond to the possible fit of observation and experiments using (4.1). (C) Numerical simulation of the collapse of granular masses with different initial shapes of the released mass (rectangular box, inclined, parabolic scar) over 2D topography performed with different friction angles (modified from Lucas et al. (2011)).

Calculating the effective friction from field data using (4.1) or using numerical simulation based on thin-layer depth-averaged model shows a clear decrease of the effective friction with increasing volume of the flowing mass (Figure 11 and Lucas et al. (2014)). A distinct trend is observed despite the very different nature and location of the investigated landslides that occur in wet or dry environments on planets with or without atmosphere. Most of the numerical analyses of individual landslides in the literature lead to calibrated friction coefficient in agreement with Figure 11 (e. g. Hungr and Evans (1996); Kelfoun and Druitt (2005), Pirulli and Mangeney (2008)). The numerical models can be viewed here as an empirical tool making it possible to recover the effective friction while taking into account the geometry of the released mass, the dynamics of the flow (spreading, 
sliding, etc.) and the topography effects. It represents a much better estimate of the effective friction than the H/L Heim's ratio widely discussed in the literature (e. g. Legros (2002), Lucas et al. (2014) and references therein). Even though the origins of this friction weakening with volume (or with other related properties such as velocity, shear rate, granular temperature, fluids, flash-heating, etc.) are still open issues, the proposed physical processes (e. g. Campbell et al. (1995), Brodu et al. (2015), Johnson et al. (2016)) should be quantitatively compatible with these field observations.

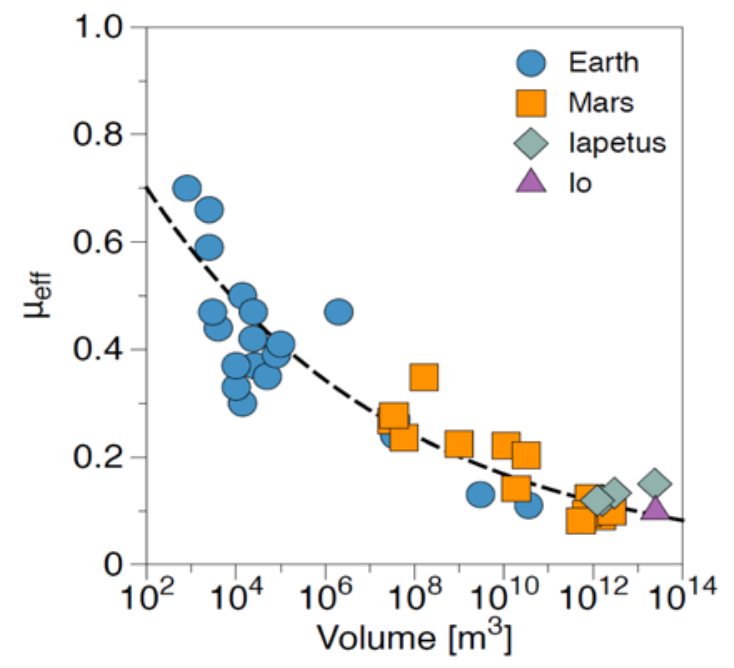

Figure 11: Effective friction $\mu_{\text {eff }}$ calculated from (4.1) for well constrained landslide on Earth and on the planets Mars, Iapetus and Io as a function of their volume $V$ for the well constrained landslides. The dashed black curve represents the empirical fit $\mu_{\text {eff }}=V^{-0.0774}$. The scatter of the data for $\mu_{\text {eff }}(V)$ is significantly smaller than when considering the Heim's ratio for the same data (adapted from Lucas et al. (2014)). A similar trend is observed when fitting the effective friction in numerical modelling of these landslides over 3D topography in order to reproduce the observed deposit extend (Lucas et al. (2014)).

4.2.2 Physical processes involved. Going further than just comparing experimental or numerical deposit extent with field observation is very challenging. In particular, all the results described in the previous section are based on comparison between characteristics of experimental, simulated and observed deposits that represent only the final flow stage. However, deposits can be reproduced with models that do not accurately describe the flow dynamics. The effect of friction weakening laws or of other physical processes such as pore fluid pressure or substrate erosion that may significantly increase the flow runout or generate surges should be constrained with 'dynamic' flow data such as stress and pressure gages or seismic data as discussed in section 4.1. Figure 12 illustrates how seismic records in different frequency ranges can be used to extract information on the interaction between the flow and the topography. This interaction is strongly related to the flow rheology and to the physical processes involved (presence of fluid, entrainment, etc.). As an example, combined seismic data analysis and numerical modelling of 200 rockfalls and pyroclastic flows in the same volcanic environment showed that taking into account friction weakening with increasing volume is necessary to reproduce seismic observations (Levy et al. (2015)). Recent laboratory experiments on acoustic waves generated by granular column collapses also show how the seismic energy reflects the different flow regimes. Such experiments permit to quantify the balance between loss of potential energy, frictional work and seismic energy (Farin et al. (2015, 2016), Farin (2015)). Another interesting example is the dynamic measurement of the position of the static-flowing interface within an erodible bed for snow avalanches (Sovilla et al. (2006, 2010)). The temporal variation of this interface is closely related to the rheology of the flow and substrate material involved (e. g. Lusso et al. (2015a)). Quantitative comparison with such measurements would make it possible to identify the difference between erosion rate with and without the presence of interstitial fluids. Indeed, laboratory experiments on dry granular flows showed that even without fluids, the presence of an erodible bed can significantly affect the flow dynamics and deposit (e. g. Mangeney et al. (2010), Farin et al. (2014)). 
(A) Inverted and simulated force filtered $20 \mathrm{~s}-80 \mathrm{~s}$
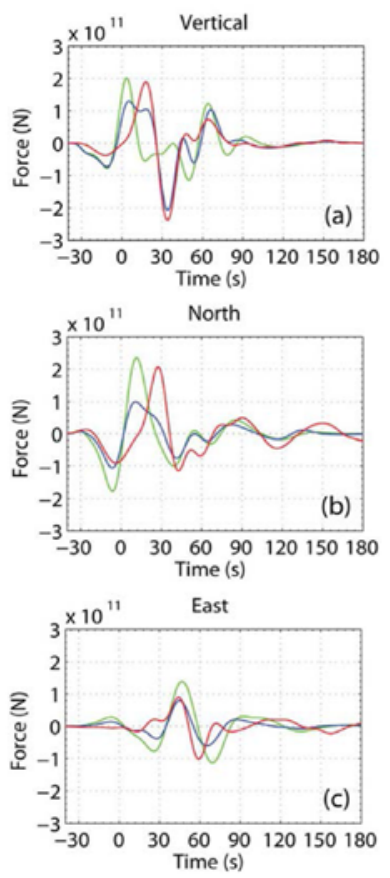

(B) Seismic amplitude filtered below $10-20 \mathrm{~s}$ and simulated momentum and work rate

(a)

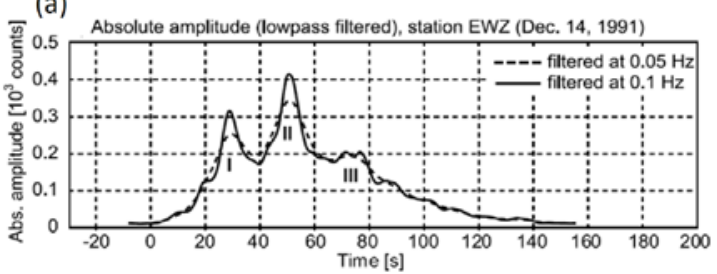

(b)

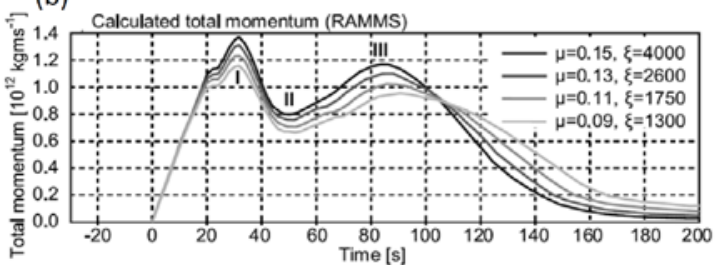

(c)

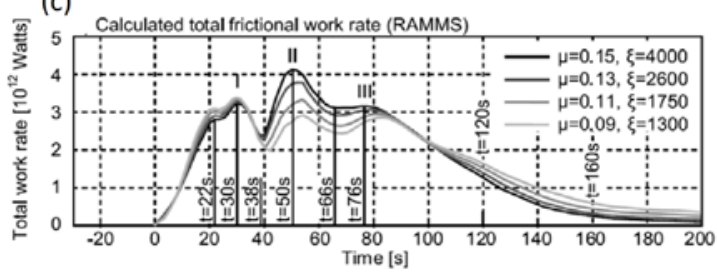

(C) Seismic energy filtered above $1 \mathrm{~Hz}$ and simulated force and work rate
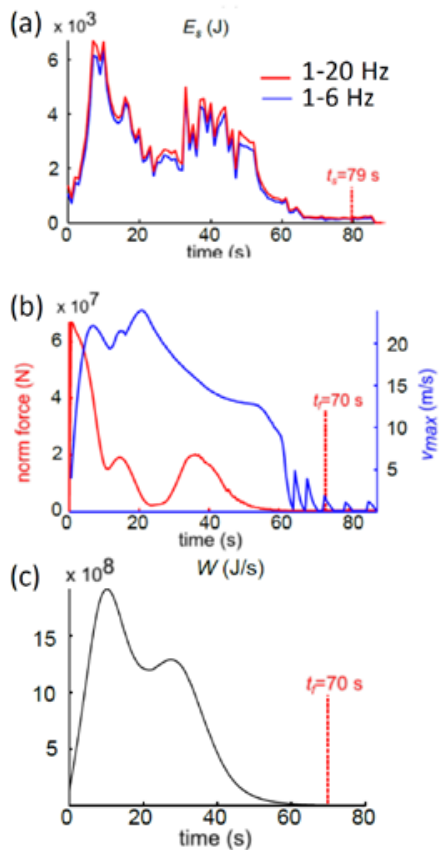

Figure 12: Comparison between low and high frequency seismic data and numerical modelling of landslides makes it possible to constrain the flow dynamics and the rheological parameters involved. (A) Comparison between the three components (a-c) of the force inverted from the low frequency (20 s-80 s) seismic signal (red line) and the simulated force constrains landslide scenarios for the 40-60 $\mathrm{Mm}^{3}$ Mount Steller rock-ice avalanche, Alaska; The blue line, representing the simulated force when erosion is taken into account, better fits the inverted force than the green line for which erosion is neglected (modified from Moretti et al. (2012)). (B) The seismic amplitude at long period (> $10 \mathrm{~s}$ ) correlates well with the frictional work rate simulated with the model RAMMS for the $6.2 \mathrm{Mm}^{3}$ Iliamna Red Glacier 2003 Avalanche in Alaska (modified from Schneider et al. (2010)). (C) The high frequency seismic energy (above $1 \mathrm{~Hz}$ ) correlates well with the simulated force for rockfalls recorded on Soufriere Hills volcano, Montserrat (modified from Levy et al. (2015)); (a) shows the seismic energy $E_{\text {s }}$ recorded for a rockfall of duration $\sim 80 \mathrm{~s}$, (b) presents the force and the maximum velocity $v_{\max }$ simulated with the SHALTOP model and (c) shows the simulated work rate $W$. For all the cases $\mathrm{A}, \mathrm{B}$, C, the time variations in the seismic data are strongly related to the interaction of the flow with topography changes along its path.

As described in section 4.1, a lot of detailed data on natural deposits (morphometric characteristics, size, shape and nature of the grains, etc.) are commonly available. Let us discuss below a few examples of the use of laboratory experiments to interpret these field data and highlight the related issues. As described in section 3, laboratory experiments and their simulation have shown that neither grains polydispersity nor fluid effects are necessary to create self-channeling flows and levees-channel deposit, while these processes change the detailed shape of the flow and deposit. This has strong implication for planetary science and in particular on Mars (e. g. Mangold et al. (2003, 2010)) where the levee-channel landslide morphologies were first interpreted as indicating the presence of water during emplacement (Malin and Edgett (2000)). Application to natural pyroclastic or submarine flows of the scaling laws described in section 3 relating the morphometric characteristics of the leveechannel deposit to the flow velocity and rheological properties may help to constrain the flow dynamics during emplacement (e. g. Jessop et al. (2012), Cannat et al. (2013)). Based on this approach, Jessop et al. (2012) suggest that the quasi-static lateral borders of the Lascar pyroclastic flows behaved like dry granular material whereas within the central channel the flow had a high pore fluid pressure. The physical processes (particle segregation, pore fluid pressure, etc.) that may lead to these differential behaviours are well explained by laboratory experiments and by their simulations as described in section 3 . There is however a strong scattering in the scaling laws derived from natural data because the slope of the topography changes from more than $20^{\circ}$ to less than $10^{\circ}$ along the path of each pyroclastic flow, the flux that generated these flows is expected to change in time, and erosion 
of the substrate made of former deposits probably occurred. Even in the same geological setting, it is difficult to compare the detailed morphological features or composition of a flow with another to infer general flow behaviour because flows generally travel on different paths and were probably generated by different upslope conditions (e. g. Brand et al. (2014)). Other morphological features could be interpreted using laboratory experiments and/or numerical modelling. As an example, the large scale abrupt change in deposit thickness observed in the Socompa debris avalanche deposit in Chile (Kelfoun and Druitt (2005)) or on the Skollahvilft snow avalanche in Iceland (Cui et al. (2007)) were shown to be related to the deflection of the flow by the local topography that generated a wave that froze when the avalanche stopped. These studies improve the understanding and prediction of maximum runup height on topographies or dams, with strong implication for hazard assessment and design of deflecting dams.

An open issue is the origin of the hummocky surface of large debris avalanches (Figure 1c). The question as to whether they may result from flow instability or waves such as those described in section 3.5 has not been investigated in detail yet. Laboratory experiments, in particular, can provide fundamental insights into flow-substrate interaction processes. Experiments by Roche et al. (2013b) have reproduced the counterintuitive modes of interaction between pyroclastic flows and horizontal granular substrates. Field observations show that pyroclastic flows whose matrix consists of ash of size less than $2 \mathrm{~mm}$ are commonly unable to rework unconsolidated substrates of fine particles of similar grain size whereas they can entrain large blocks commonly larger than $10 \mathrm{~cm}$ in diameter (Roche et al. (2016)). The experiments reveal that a flow actually slides on a finely grained substrate. In contrast in case of a substrate of coarse grains the flow particles first penetrate into the substrate interstices and basal shear promotes extraction of the grains. Substrate-derived particles are then first dragged slowly at flow base before being uplifted because of a dynamic pressure gradient at the flowsubstrate interface that scales with the square of the flow velocity. In case a granular substrate is inclined, experiments by Rowley (2011) and Farin et al. (2014) show that shear imposed by the flow on the underlying material generates features that resemble Kelvin-Helmholtz instabilities typical of pure fluids. The material entrained from the substrate can then be mixed thoroughly with the flow particles and may represent a significant proportion of the basal part of the deposits.

\section{Conclusions and outlook}

There are essentially four ways to capture the physics of granular and biphasic geophysical flows: field studies, large-scale experiments, laboratory experiments and numerical simulations. Some of these routes have experienced significant progress due in particular to improved measurement techniques and imaging, as well as increased computing capabilities. However, progress remains limited due to difficulties in modeling and understanding the mechanisms that govern these flows. We end this review by highlighting some issues where improvements can be expected.

Starting with the most basic aspects, a problem arises as soon as one has to specify the material properties of the grains, including particularly restitution and friction coefficients. They are of great importance for numerical simulations using discrete elements, for example. These coefficients are intended to account for complex behaviors at the nanometer scale. It is well known that the restitution coefficient is actually not an intrinsic material property, since it also depends on the grain speed at impact (Goldsmith (1960)). The friction coefficient also has a complex behavior: we know that the static friction coefficient is subject to aging effects and that it usually takes a slightly larger value than the dynamic friction coefficient. It is perhaps less known that the friction coefficient measured in a binary collision often takes much smaller values (Louge and Keast (2001)). The friction coefficient is thus not a trivial physical characteristic and its dependence on the nature of the contact interaction between grains is poorly documented. In the absence of a detailed understanding and description of contact interaction, it is difficult to take these dependences into account in DEM type simulations. The pertinent choice of the values of these coefficients in simulations therefore remains an important issue. Other problems concerning the modeling of contact interactions arise also from the shape of the grains (e.g., grains with angular edges), from cohesion forces induced by electromagnetic interactions (e.g., electrostatic and Van der Waals forces) or by capillarity when grains are surrounded by both air and water, and from interstitial pore fluid pressure that damps interactions. Fragmentation of the grains may also, of course, change their impact properties. 
The description of the interactions between the grains and the fluid that surrounds them also raises important issues. Unsteady effects (such as the "added mass" for example) are not taken properly into account yet. Similarly, at a larger scale, in continuum descriptions, some collective effects are not always well considered. For example, the damping of the fluid turbulence by the presence of the particle is poorly understood and documented.

In general, change of scales (e.g., the passage from the grain scale to the meso-scale - e.g., laboratory scale- or from the meso-scale to the large spatial scale of the natural phenomena) is another important issue. The kinetic theory provides an ab initio framework to move from a discrete to a continuum description, but it is relevant only for flows dominated by collisional interactions, which are generally dilute. For flow regimes in which persistent contacts prevail - generally dense flows only continuum phenomenological models are available and are often restricted to unidirectional flows. There are only very few attempts to connect these two types of models for flows in which quasi-static and flowing regions coexist and where a transition between these two "states" is possible. We do not know either how to describe the transition from motion to rest (unjamming/jamming transition). Nevertheless, this description is crucial for particle-laden flows, since most of the information we have about the flow is its deposit.

These issues on transition between static and dynamic regimes raise the problem of boundary conditions that are often difficult to specify, either in terms of geometry or energy. We do not have a model capable of describing all flow regimes and of taking into account properly the boundary conditions, even in the simple case of mono-disperse and unidirectional flows. Moreover, if we consider (i) the natural size dispersion (often very large in natural flows) which induces important changes in local size distribution by segregation effect, (ii) the evolution of the particle size distribution due to fragmentation (which also have an impact on the energy of the flow), (iii) the effects of cohesion between grains and (iv) the three-dimensional nature of the flows (partly related to changes in slope and terrain), it appears that we are very far from being able to model a natural flow in all its complexity.

For progressing in this modeling work, experimental studies are extremely valuable. The ideal approach would be to document in situ natural phenomena in real time. Unfortunately, this is rarely possible due to the unpredictability (in time and space) of these events. When there is a high probability of occurrence in a given place (e.g. snow avalanches), there is also the difficulty of measuring in unstable places with hostile conditions for life and material. For these reasons, it is essential to conduct experiments in the laboratory but also at larger scale by either constructing large devices or triggering flows in natural areas previously instrumented. The use of such large-scale experiments is necessary when there is no known similarity (or scaling) that allows extrapolating the results at the laboratory scale to the field scale. Some phenomena may also appear only beyond a threshold that may be impossible to achieve in the laboratory (e.g., very high flow rate). The development of measurement methods for accessing the flow properties such as the grain speed, the grain concentration, or the stresses that develop within the flow or at the boundaries is a key element for improving the knowledge on natural flows. Currently efficient sensors for measuring strain and new methods are emerging to measure the particle concentration in dense suspensions. Other promising technologies are under development: acoustic methods as well as capacitive, gravity or seismic measurements. Some of these methods (seismic methods for example) can also be used in the field as the sensors receive the signal from long distance and therefore do not need to be installed in advance in dangerous and unpredictable places. However, these methods require a lot of development to interpret the signal and to link it with the underlying physical phenomena.

All these considerations show that measurements and modeling are also closely related. Quantitative evaluation of the one or the other, as well as the change of scale (from laboratory to field scale), require detailed comparison using numerical simulations. A qualitative comparison is insufficient. A quantitative comparison is the only way to emphasize the limitations of the models and to identify their missing physical processes. This requires the development of innovative tools such as the inverse methods for example, since measurements usually report incomplete data on properties of the natural phenomena. This also calls for the collection of field and laboratory data acquired all over the world to perform comparisons with numerical simulations in an open and collaborative manner between different scientific communities (e.g., earth science, physics, applied mathematics and computing science). This sharing of knowledge would perhaps also raise the interest of these studies 
and models among institutions responsible for the evaluation of natural hazards and risks posed by geophysical mass flows.

\section{References}

Aguirre M A, Nerone N, Calvo A, Ippolito I and Bideau D 2000 Influence of the number of layers on the equilibrium of a granular packing Phys. Rev. E 62738

Aguirre M A, Nerone N, Ippolito I, Calvo A and Bideau D 2001 Granular packing: influence of different parameters on its stability Granular Matter $\mathbf{3} 75$

Ahn H, Brennen C E and Sabersky R H 1991 Measurements of Velocity, Velocity Fluctuation, Density, and Stresses in Chute Flows of Granular Materials Journal of Applied Mechanics 58 792-803

Allstadt K 2013 Extracting Source Characteristics and Dynamics of the August 2010 Mount Meager Landslide from Broadband Seismograms J. Geophys. Res. Earth Surface 118 1472-1490.

Ancey C 2001 Dry granular flows down an inclined channel: Experimental investigations on the frictionalcollisional regime Physical Review E 65011304.

Ancey C and Heyman J 2014 A microstructural approach to bed load transport: mean behaviour and fluctuations of particle transport rates Journal of Fluid Mechanics 744 129-168

Anderson T B and Jackson R 1967 A fluid mechanical description of fluidized beds: equations of motion Ind. Eng. Chem. Fundam. 6 527-539

Anderson K, Sundaresan S and Jackson R 1995 Instabilities and the formation of bubbles in fluidized beds $J$. Fluid Mech. 303 327-366

Aradian A, Raphaël E and de Gennes P-G 2002 Surface flows of granular materials: a short introduction to some recent model C.R. Physique 3 187-196

Aranson I S and Tsimring L S 2002 Continuum theory of partially fluidized granular flows Phys. Rev. E 65 061303

Aranson I S, Malloggi F and Clément E 2006 Transverse instability of avalanches in granular flows down an incline Phys. Rev. E 73050302

Aranson I S, Tsimring L S, Malloggi F and Clement E 2008 Nonlocal rheological properties of granular flows near a jamming limit Phys. Rev. E 78031303

Arattano M and Marchi L 2008 Systems and sensors for debris-flow monitoring and warning Sensors 8 24362452

Armanini A, Capart H, Fraccarollo L and Larcher M 2005 Rheological stratification in experimental free-surface flows of granular-liquid mixtures J. Fluid Mech 532 269-319

Armanini A, Larcher M and Fraccarollo L 2009 Intermittency of rheological regimes in uniform liquid-granular flows Physical Review E 79051306

Artoni R, Santomaso A and Canu P 2009 Effective boundary conditions for dense granular flows Physical Review E 79031304.

Artoni R, Santomaso A C and Canu P 2012 Scaling Laws for the Slip Velocity in Dense Granular Flows Phys. Rev. Lett.108 238002

Aussilous P, Chauchat J, Pailha M, Médale M and Guazzelli E 2013 Investigation of the mobile granular layer in bedload transport by laminar shearing flows J. Fluid Mech. 736 594-615.

Azanza E, Chevoir F and Moucheront P 1999 Experimental Study of Collisional Granular Flows Down an Inclined Plane Journal of Fluid Mechanics 400 199-227

Bagnold R A 1954 Experiments on a gravity-free dispersion of large solid spheres in a Newtonian fluid under shear Proc. R. Soc. Lond. A. 225 49-63

Balmforth N J and Kerswell R R 2005 Granular collapse in two dimensions Journal of Fluid Mechanics 538 $399-428$

Baker J L, Barker T and Gray J M N T 2016 A two-dimensional depth-averaged-rheology for dense granular avalanches Journal of Fluid Mechanics 787 367-395

Barker T, Schaeffer D G, Bohorquez P and Gray J M N T 2015 Well-posed and ill-posed behaviour of the mu(I)rheology for granular flow Journal of Fluid Mechanics 779 794-818

Bedford A and Drumheller D S 1983 Recent advances: theories of immiscible and structured mixtures. Int. J. Eng. Sci. 21 863-960

Benito J, Bertho Y, Ippolito I and Gondret P 2012 Stability of a granular layer on an inclined "fakir plane" EuroPhys. Lett. 10034004

Berger C, McArdell BW and Schlunegger F 2011 Direct measurement of channel erosion by debris flows, Illgraben, Switzerland J. Geophys. Res. Earth Surface 116 F1 2156-2002

Berzi D and Jenkins J T 2008 A theoretical analysis of free-surface flows of saturated granular-liquid mixtures J Fluid Mech. 608 393-410 
Berzi D, Jenkins J T and Larcher M 2010 Debris flows: Recent advances in experiments and modeling Advances in Geophysics 52 103-138

Bi W, Delannay R, Richard P, Taberlet N and Valance A 2005 Two-and three-dimensional confined granular chute flows: experimental and numerical results Journal of Physics: Condensed Matter 17 S2457-S2480

Bi W, Delannay R, Richard P and Valance A 2006 Experimental study of two-dimensional, monodisperse, frictional-collisional granular flows down an inclined chute Physics of Fluids 18123302

Bingham E C 1922 Fluidity and Plasticity (New York, McGraw-Hill)

Bolthenhagen P 1999 Boundary effects on the maximal angle of stability of a granular packing Eur. Phys. J. B 1275

Börzsönyi T, Halsey T C and Ecke R E 2005 Two scenarios for avalanche dynamics in inclined granular layers Physical review letters 94208001.

Börzsönyi T, Halsey T C and Ecke R E 2008 Avalanche dynamics on a rough inclined plane Physical Review E 78011306

Börzsönyi T, Ecke R E and McElwaine J N 2009 Patterns in flowing sand: Understanding the physics of granular flow Phys. Rev. Lett. 103178302

Bouchaud J-P, Cates M E, Prakash J R and Edwards S F 1994 A model for the dynamics of sandpile surfaces Journal de Physique I 4 1383-1410

Bouchut F, Mangeney-Castelnau A, Perthame B and Vilotte J-P 2003 A new model of Saint-Venant and SavageHutter type for gravity driven shallow water flows Comptes Rendus Mathematique 336 531-536

Bouchut F and Westdickenberg M 2004 Gravity driven shallow water models for arbitrary topography Commun. Math. Sci. 2 359-389

Bouchut F, Fernàndez-Nieto E, Mangeney A and Lagrée P-Y 2008 On new erosion models of savage-hutter type for avalanches Acta Mechanica 199 181-208

Bouchut F, Fernandez-Nieto E D, Mangeney A and Narbona-Reina G 2015a A two-phase shallow debris flow model with energy balance Math. Model. Num. Analy. 49 101-140.

Bouchut F, Fernandez-Nieto E D, Mangeney A and Narbona-Reina G 2015b A two-phase two-layer model for fluidized granular flows with dilatancy effects J. Fluid Mech. 199 181-208

Bouchut F, Ionescu I and Mangeney A 2016 An analytic approach for the evolution of the static-flowing interface in viscoplastic granular flows Comm. Math. Sci. in press

Boutreux T, Raphaël E and de Gennes P G 1998 Surface flows of granular materials: a modified picture for thick avalanches Physical Review E 584692.

Boyer F, Guazzelli E and Pouliquen O 2011 Unifying Suspension and Granular Rheology Physical Review Letter 107188301

Branney M J and Kokelaar B P 2002 Pyroclastic density currents and the sedimentation of ignimbrites Geological Society of London, Memoir 27

Brand B D, Mackaman-Lofland C, Pollock N M, Bendaña S, Dawson B and Wichgers P 2014 Dynamics of pyroclastic density currents: Conditions that promote substrate erosion and self-channelization - Mount St Helens, Washington (USA) J. Volcano. Geotherm. Res. 276 189-214.

Brodu N, Delannay R and Richard P 2013 Shallow granular flows down flat frictional channels: Steady flows and longitudinal vortices Physical Review E 87022202

Brodu N, Delannay R, Valance A and Richard P 2015 Evidence for new regimes in channeled granular flows Journal of Fluid Mechanics 769 218-228

Brodsky E E, Gordeev E and Kanamori H 2003 Landslide basal friction as measured by seismic waves Geophys. Res. Lett. 302236.

Brufau P, Garcia-Navarro P, Ghilardi P, and Natale P 2000 1-d mathematical modelling of debris flow $J$. Hydraul. Res. 38 435-446

Calder E S, Sparks R S J and Gardeweg M C 2000 Erosion, transport and segregation of pumice and lithic clasts in pyroclastic flows inferred from ignimbrite at Lascar Volcano, Chile Journal of Volcanology and Geothermal Research 104 201-235

Calder E S, Luckett R, Sparks R S J and Voight B 2002 Mechanisms of lava dome instability and generation of rockfalls and pyroclastic flows at Soufriere Hills Volcano, Montserrat Geological Society, London, Memoirs 200221 173-190

Campbell C, Clearly P W and Hopkins M 1995 Large-scale landslide simulations: Global deformation, velocities and basal friction J. Geophys. Res. $1008267-8283$

Cannat M, Mangeney A, Ondréas H, Fouquet Y and Normand A 2013 High- resolution bathymetry reveals contrasting landslide activity shaping the walls of the Mid-Atlantic Ridge axial valley Geochemistry, Geophysics, Geosystems 14 996-1011

Cantero M I, Cantelli A, Pirmez C, Balachandar S, Mohrig D, Hickson T A and Parker G 2012 Emplacement of massive turbidites linked to extinction of turbulence in turbidity currents Nature Geoscience 5 42-45 
Capart H, Hung C-Y and Stark C P 2015 Depth-integrated equations for entraining granular flows in narrow channels Journal of Fluid Mechanics 765 R4

Cappa F, Guglielmi Y, Viseur S and Garambois S 2014 Deep fluids can facilitate rupture of slow-moving giant landslides as a result of stress transfer and frictional weakening Geophysical Research Letters 41 1-6

Carneiro M V, Pähtz T, and Herrmann H J 2011 Jump at the onset of saltation Phys. Rev. Lett. 107098001

Cassar C, Nicolas M and Pouliquen O 2005 Submarine granular flows down inclines planes Physics of Fluids 17 103301

Chen C L and Ling C H 1998 Rheological equations in asymptotic regimes of granular flows Journal of engineering mechanics 124 301-310

Chigira M, Wang W N, Furuya T and Kamai T 2003 Geological causes and geomorphological precursors of the Tsaoling landslide triggered by the 1999 Chi-Chi earthquake, Taiwan Engineering Geology 68 259-273

Conway S J, Decaulne A, Balme M R, Murray J B and Towner M C 2010 A new approach to estimating hazard posed by debris flows in the Westfjords of Iceland Geomorphology 114 556-572

Cortet P-P, Bonamy D, Daviaud F, Dauchot O, Dubrulle B and Renouf M 2009 Relevance of visco-plastic theory in a multi-directional inhomogeneous granular flow Europhysics Letters 8814001

Courrech du Pont S, Gondret P, Perrin B and Rabaud M 2003a Granular avalanches in fluids Phys. Rev. Lett. 90 044301

Courrech du Pont S, Gondret P, Perrin B and Rabaud M 2003b Wall effect on granular heap stability Europhysics Letters 61 492-498

Coussot P 1994 Steady laminar flow of concentrated mud suspensions in open channel J. Hydraul. Res. 32 535559

Coussot P and Meunier M 1996 Recognition, classification and mechanical description of debris flows EarthScience Reviews 40 209-227

Crosta G B, Imposimato S, Roddeman Wright D, Yang R Y, Xu B H and Yu A B 2009 Numerical modeling of 2-D granular step collapse on erodible and non-erodible surface J. Geophys. Res. 114 F03020

Cui X, Gray J M N T and Johannesson T 2007 Deflecting dams and the formation of oblique shocks in snow avalanches at Flateyri, Iceland Journal of Geophysical Research 112 F04012

Cui X and Gray J M N T 2013 Gravity-driven granular free-surface flow around a circular cylinder Journal of Fluid Mechanics 720, 314-337

Cundall P and Strack O 1979 A discrete numerical model for granular assemblies Geotechnique 29 47-65

Da Cruz F, Emam Prochnow S M, Roux J-N and Chevoir F 2005 Rheophysics of dense granular materials: discrete simulation of plane shear flows Phys. Rev. E 72021309

Daerr A 2001 Dynamical equilibrium of avalanches on a rough plane Physics of Fluids 13 2115-2124

Dallavalle J 1943 Micrometrics (New York: Pitman)

Dammeier F, Moore J R, Haslinger F and Loew S 2011 Characterization of alpine rockslides using statistical analysis of seismic signals J. Geophys. Res. 116 F04024

De Blasio F V and Elverhøi A 2008 A model for frictional melt production beneath large rock avalanches $J$. Geophys. Res. 113 F02014

De Blasio F V and Crosta G B 2014 Simple physical model for the fragmentation of rock avalanches Acta Mecha. 225 243-252

Deboeuf S, Dauchot O, Staron L, Mangeney A and Vilotte J P 2005 Memory of the Unjamming Transition during Cyclic Tiltings of a Granular Pile Phys. Rev. E 72051305

Deboeuf S, Lajeunesse E, Dauchot O and Andreotti B 2006 Flow Rule, Self-Channelization, and Levees in Unconfined Granular Flows Phys. Rev. Lett. 97158303

Delannay R, Louge M, Richard P, Taberlet N and Valance A 2007 Towards a theoretical picture of dense granular flows down inclines Nature Materials 6 99-108

Denlinger R P and Iverson R M 2001 Flow of variably fluidized granular masses across three-dimensional terrain 2. Numerical predictions and experimental tests J. Geophys. Res. 106 553-566

Denlinger R P and Iverson R M 2004 Granular avalanches across irregular three-dimensional terrain: 1. Theory and computation J. Geophys. Res. 109 F01014

Deparis J, Jongmans D, Cotton F, Baillet L, Thouvenot F and Hantz D 2007 Analysis of rock-fall seismograms in the western Alps Bull. Seismol. Soc. Am. 98 1781-1796

DeRoin N and McNutt S R 2012 Rockfalls at Augustine Volcano, Alaska: The influence of eruption precursors and seasonal factors on occurrence patterns 1997 J. Volcano. Geotherm. Res. 211 61-75

Doyle E E, Huppert H E, Lube G, Mader H M and Sparks R S J 2007 Static and flowing regions in granular collapses down channels: Insights from a sedimenting shallow water model Physics of Fluids 19106601

Doyle E E, Cronin S J and Thouret J-C 2011 Defining conditions for bulking and debulking in lahars Geological Society of America Bulletin 123 1234-1246

Drew D 1983 Mathematical modeling of two-phase flow Ann. Rev. fluid Mech. 15 261-291

Druitt T H 1998 Geological Society, London, Special Publications 145 145-182. 
Dunning S A, Mitchell W A, Rosser N J and Petley D N 2007 The Hattian Bala rock avalanche and associated landslides triggered by the Kashmir Earthquake of 8 October 2005 Engineering Geology 93 130-144

Duran O, Andreotti B and Claudin P 2012 Numerical simulation of turbulent sediment transport from bed load to saltation Phys. Fluids 24103306

Dufek J 2016 The fluid mechanics of pyroclastic density currents Annu. Rev. Fluid Mech.48 459-485

Edwards A N and Gray J M N T 2015 Erosion-deposition waves in shallow granular free-surface flows J. Fluid Mech. 762 35-67

Eggenhuisen J T, McCaffrey W D, Haughton P D W and Butler R W H 2011 Shallow erosion beneath turbidity currents and its impact on the architectural development of turbidite sheet systems. Sedimentology 58 936959

Ekström G and Stark C P 2013 Simple scaling of catastrophic landslide dynamics Science 339 1416-1419

Ertas D G. Grest S, Halsey T C, Levine D and Silbert L E 2001 Gravity-driven dense granular flows Europhys. Lett. 56 214-220

Faccanoni G and Mangeney A 2013 Exact solution for granular flows Int. J. Num. Analy. Meth. Geomech. 37 1408-1433

Farin M, Mangeney A and Roche O 2014 Dynamics, deposit and erosion processes in granular collapse over sloping beds J. Geophys. - Earth Surface 119 504-532

Farin M 2015 Etudes expérimentales de la dynamique et de l'émission sismique des instabilités gravitaires Ph.D. thesis IPGP Paris

Farin M, Mangeney A, Toussaint R, De Rosny J, Shapiro N, Dewez T, Hibert C, Mathon C, Sedan O and Berger F 2015 Characterization of rockfalls from seismic signal: insights from laboratory experiments J. Geophys. Res. Solid Earth 120 7102-7137

Farin M, Mangeney A, De Rosny J, Toussaint R, Sainte-Marie J and Shapiro N 2016 Experimental estimation of the elastic energy radiated during an impact J. Sound Vibration 362 176-202

Faug T, Gauer P, Lied K and Naaim M 2008 Overrun length of avalanches overtopping catching dams: crosscomparison of small-scale laboratory experiments and observations from full-scale avalanches J. Geophys. Res. 113 F03009

Favreau P, Mangeney A, Lucas A, Crosta G and Bouchut F 2010 Numerical modeling of landquakes Geophys. Res. Lett. 37 L15305

Félix G and Thomas N 2004 Relation between dry granular flow regimes and morphology of deposits: formation of levées in pyroclastic deposits Earth and Planetary Science Letters 221 197-213

Fernandez-Nieto E, Bouchut F, Bresch D, Castro-Diaz M J and Mangeney A 2008 A new Savage-Hutter type model for submarine avalanches and generated tsunami J. Comp. Phys. 227 7720-7754

Fernandez-Nieto E D, Garres-Diaz J, Mangeney A and Narbona-Reina G 2016 A multilayer shallow model for dry granular flows with the mu(I) rheology: Application to granular collapse on erodible beds J. Fluid Mech. accepted

Fischer R, Gondret P, Perrin B and Rabaud M 2008 Dynamics of dry granular avalanches Phys. Rev. E 78 021302

Forterre Y and Pouliquen O 2001Longitudinal vortices in granular fl ow Phys. Rev. Lett. 86 5886-5889

Forterre Y and Pouliquen O 2003Long-surface-wave instability in dense granular flows Journal of Fluid Mechanics 486 21-50

Forterre Y 2006 Kapiza waves as a test for three-dimensional granular flow rheology. Journal of Fluid Mechanics 563 123-132

Frette V, Christensen K, The-Sorenssen A M, Feder J, Jossang T and Meakin P 1996 Avalanche dynamics in a pile of rice Nature 379 49-52

Gauer P and Issler D 2004 Possible erosion mechanisms in snow avalanches Annals of Glaciology 38 384-392

GDR-MiDi 2004 On dense granular flows Eur. Phys. J. E 14 341-365

Girolami L, Druitt T H, Roche O and Khrabrykh Z 2008 Propagation and hindered settling of laboratory ash flows Journal of Geophysical Research 113 B02202

Girolami L, Roche O, Druitt T H and Corpetti T 2010 Velocity fields and depositional processes in laboratory ash flows Bulletin of Volcanology 72 747-759

Girolami L, Hergault V, Vinay G and Wachs A 2012 A three-dimensional discrete-grain model for the simulation of dam-break rectangular collapses: comparison between numerical results and experiments Granular Matter 14 381-392

Gladstone C and Sparks R S J 2002 The significance of grain-size breaks in turbidites and pyroclastic density current deposits Journal of Sedimentary Research 72 182-191

Goldsmith W 1960 Impact - The Theory and Behaviour of Colliding Solids (London: Edward Arnold Ltd.)

Gondret P, Lance M and Petit L 2002 Bouncing motion of spherical particles in fluids Physics of Fluids 14 643651 
Goren L and Aharonov E 2007 Long runout landslides: the role of frictional heating and hydraulic diffusivity Geophysical research letters 34 L07301

Goren L, Aharonov E and Anders M H 2010 The long runout of the Heart Mountain landslide: Heating, pressurization, and carbonate decomposition J. Geophys. Res. 115 B10210

Goujon C, Thomas N and Dalloz-Dubrujeaud B 2003 Monodisperse dry granular flows on inclined planes: Role of roughness The European Physical Journal E: Soft Matter and Biological Physics 11 147-157

Goujon C, Dalloz-Dubrujeaud B and Thomas N 2007 Bidisperse granular avalanches on inclined planes: a rich variety of behaviors Eur. Phys. J. E 23 199-215

Gray J M N T \& Hutter K 1997 Pattern formation in granular avalanches Continuum Mechanics and Thermodynamics 9 341-345

Gray J M N T, Wieland M and Hutter K 1999 Gravity driven free surface flow of granular avalanches over complex basal topography Proc. R. Soc. London Ser. A 455 1841-1874

Gray J M N T 2001 Granular flow in partially filled slowly rotating drums J. Fluid Mechanics 441 1-29

Gray J M N T, Tai Y C and Noelle S 2003 Shock waves, dead-zones and particle-free regions in rapid granular free-surface flows J. Fluid Mechanics 491 161-181

Gray J M N T and A R Thornton 2005 A theory for particle size segregation in shallow granular free-surface flows Proc. R. Soc. A 461 1447-1473

Gray J M N T and Cui X 2007 Weak, strong and detached oblique shocks in gravity driven granular free-surface flows J. of Fluid Mechanics 579 113-136

Gray J M N T and Kokelaar B P 2010 Large particle segregation, transport and accumulation in granular freesurface flows J Fluid Mech 652 105-137

Gray J M N T and Ancey C 2011 Multi-component particle-size segregation in shallow granular avalanches $J$. Fluid Mech. 678 535-588

Gray J M N T and Edwards A N 2014 A depth-averaged-rheology for shallow granular free-surface flows $J$. Fluid Mech. 755 503-534

Grigorian S S, Eglit M E, Iakimov I L 1967 New statement and solution of the problem of the motion of snow avalanche. Snow, Avalanches \& Glaciers Tr. Vysokogornogo Geofizich Inst. 12 104-13

Haas F, Heckmann T, Wichmann V and Becht M 2012 Runout analysis of a large rockfall in the Dolomites/Italian Alps using LIDAR derived particle sizes and shapes Earth Surf. Process. Landforms 37 1444-1455

Hákonardóttir K M and Hogg A J 2005 Oblique shocks in rapid granular flows Phys. Fluids 170077101

Hallworth M A, Phillips J C, Huppert H E and Sparks R S J 1993 Entrainment in turbulent gravity currents Nature 362 829-831

Hampton M A, Lee H J and Locat J 1996 Submarine landslides Reviews of Geophysics 34 33-59

Hayashi J N and Self S 1992 A comparison of pyroclastic flow and debris avalanche mobility Journal of Geophysical Research: Solid Earth 97 9063-9071

Helmstetter A and Garambois S 2010 Seismic monitoring of Séchilienne Rockslide (French Alps) : analysis of seismic signals and their correlation with rainfalls Journal of Geophysical Research: Earth Surface 115 F03016

Henann D. L. and Kamrin K 2013 A predictive size-dependent continuum model for dense granular flows PNAS 110 6730-6735

Hibert C, Mangeney A, Grandjean G and Shapiro N M 2011 Slope instabilities in Dolomieu crater, Réunion Island: From seismic signals to rockfall characteristics Journal of Geophysical Research: Earth Surface 116 F04032

Hibert C, Mangeney A, Grandjean G, Baillard C, Rivet D, Shapiro N M and Crawford W 2014a Automated identification, location, and volume estimation of rockfalls at Piton de la Fournaise volcano Journal of Geophysical Research: Earth Surface 119 1082-1105

Hibert C, Ekström G and Stark C P 2014b Dynamics of the Bingham Canyon Mine landslides from seismic signal analysis Geophysical Research Letters 41 4535-4541

Hill K M, and Yohannes B 2011 Rheology of dense granular mixtures: Boundary pressures Phys. Rev. Lett. 106 058302

Hill K M and Tan D S 2014 Segregation in Dense Sheared Flows: Gravity, Temperature Gradients, and Stress Partitioning J. Fluid Mech. 756 54-88

Hill K M and Fan Y 2016 Granular Temperature and Segregation in Dense Sheared Particulate Mixtures KONA Powder and Particle Journal 33 150-168

Hogg A J 2007 Two-dimensional granular slumps down slopes Physics of Fluids 19093301

Holyoake A J and McElwaine J N 2012 High-speed granular chute flows Journal of Fluid Mechanics 710 35-71

Hopfinger E J 1983 Snow avalanche motion and related phenomena Annual Review of Fluid Mechanics 15 4776 
Hsu T-J, Jenkins J T and Liu P L-F 2003 On two-phase sediment transport: dilute flow J. Geophys. Res. 108 3057

Hsu T-J, Jenkins J T and Liu P L-F 2004 On two-phase sediment transport: sheet flow of massive particles Proc. R. Soc. Lond. A 460 2223-2250

Hsü K J 1975 Catastrophis debris streams (Sturzstroms) generated by rockfalls Geological Society of America Bulletin 86 129-140

Huggel C, Zgraggen-Oswald S, Haeberli W, Kääb A, Polkvoj A, Galushkin I, Evans S G, Crosta G B, Schneider J-L and Strom A 2005 The 2002 rock/ice avalanche at Kolka/Karmadon, Russian Caucasus: assessment of extraordinary avalanche formation and mobility, and application of QuickBird satellite imagery Natural Hazards \& Earth System Sciences 5 173-187

Huggel C, Caplan-Auerbach J, Molnia B and Wessels R 2008 The 2005 Mt. Steller, Alaska, rock-ice avalanche: A large slope failure in cold permafrost Proc. of the Ninth Int. Conf. on Permafrost (Fairbanks, USA, 29 June-3 July 2008) vol. 29, ed D Kane and K M Hinkel pp. 747-752

Hungr O and Evans S G 1996 Rock avalanche runout prediction using a dynamic model Proc. of the 7th Int. Symp. on Landslides (Norway, Trondheim) (Rotterdam: Balkema) Vol. 1 pp. 233-238

Hungr O, Evans S G, Bovis M J and Hutchinson J N 2001 A review of the classification of landslides of the flow type Environmental and Engineering Geoscience 7221238.

Hungr O and Evans S G 2004 Entrainment of debris in rock avalanches: an analysis of a long run-out mechanism Geological Society of America Bulletin 116 1240-1252

Hungr O, Leroueil S and Picarelli L 2013 The Varnes classification of landslide types, an update Landslides 11 167-194

Imre B, Laue J and Springman S M 2010 Fractal fragmentation of rocks within sturzstroms: insight derived from physical experiments within the ETH geotechnical drum centrifuge Granular Matter 12 267-285

Ionescu I R, Mangeney A, Bouchut F and Roche O 2015 Viscoplastic modeling of granular column collapse with pressure-dependent rheology Journal of Non-Newtonian Fluid Mechanics 219 1-18.

Iverson R M 1997 The physics of debris flows Reviews of Geophysics 35 245-296

Iverson R M, Reid M E and LaHusen R G 1997 Debris-flow mobilization from landslides Annual Review of Earth and Planetary Sciences 25 85-138

Iverson R M, Reid M E, Iverson N R, LaHusen R G, Logan M, Mann J E and Brien D L 2000 Acute sensitivity of landslide rates to initial soil porosity Science 290 513-516

Iverson R M and Denlinger R P 2001 Flow of variably fluidized granular masses across three dimensional terrain 1. Coulomb mixture theory J. Geophys. Res. 106 537-552

Iverson R M and Vallance J W 2001 New views of granular mass flows Geology 29 115-118

Iverson R M, Logan M and Denlinger R P 2004 Granular avalanches across irregular three-dimensional terrain: 2. Experimental tests Journal of Geophysical Research 109 F01015

Iverson R M 2005 Regulation of landslide motion by dilatancy and pore pressure feedback J. Geophys. Rev. 110 F02015

Iverson R M, Logan M, LaHusen R G and Berti M 2010 The perfect debris flow? aggregated results from 28 large-scale experiments J. Geophys. Res. 115 F03005

Iverson R M, Reid M E, Logan M, LaHusen R G, Godt J W and Griswold J P 2011 Positive feedback and momentum growth during debris-flow entrainment of wet bed sediment Nature Geoscience 4 116-121

Iverson R M 2012 Elementary theory of bed-sediment entrainment by debris flows and avalanches J. Geophys.

Res. 117 F03006

Iverson R M and George D L 2014 A depth-averaged debris-flow model that includes the effects of evolving dilatancy. I. Physical basis Proc. R. Soc. A 47020130819

Iverson R M and Ouyang C 2015 Entrainment of bed material by Earth-surface mass flows: review and reformulation of depth-integrated theory Rev. Geophys. 53 27-58

Iverson R M 2015 Scaling and design of landslide and debris-flow experiments Geomorphology 244 9-20

Jaboyedoff M, Oppikofer T, Abellan A, Derron M H, Loye A, Metzger R and Pedrazzini A $2012 \quad$ Use of LIDAR in landslide investigations: a review Nat. Hazards 61 5-28

Jackson R 1997 Locally averaged equations of motion for a mixture of identical spherical particles and a newtonian fluid Ch. Engng Sci. 52 2457-2469

Jackson R 2000 The dynamics of fluidized particles (Cambridge: Cambridge University Press)

Jaeger H M, Liu C-H and Nagel S R 1989 Relaxation at the Angle of Repose Phys. Rev. Lett. 62 40-43

Jenkins J and Savage S 1983 A theory for the rapid flow of identical, smooth, nearly elastic, spherical particles. J. Fluid Mech. 130 187-202

Jenkins J T and Richman M W 1985 Grad's 13-moment system for a dense gas of inelastic spheres Archive for Rational Mechanics and Analysis, 87 355-377

Jenkins J T 1994 Rapid granular flow down inclines Appl. Mech. Rev. 47 S240-S244 
Jenkins J T and Hanes D M 1998 Collisional sheet flows of sediment driven by a turbulent fluid Journal of fluid Mech. 370 29-52

Jenkins J T 2006 Dense shearing flows of inelastic disks Physics of Fluids 18103307

Jenkins J T 2007 Dense inclined flows of inelastic spheres Granular Matter 10 47-52

Jenkins J T and Berzi D 2010 Dense inclined flows of inelastic spheres: tests of an extension of kinetic theory Granular Matter 12 151-158

Jessop D, Kelfoun K, Labazuy P, Mangeney A, Roche O, Tillier J-L, Trouillet M and Thibault G 2012 LiDAR derived morphology of the 1993 Lascar pyroclastic flow deposits, and implication for flow dynamics and rheology Journal of Volcanology and Geothermal Research 24581 - 97

Johnson P C, Nott P and Jackson R 1990 Frictional-collisional equations of motion for particulate flows and their application to chutes Journal of Fluid Mechanics 210 501-535

Johnson R W 1998 The handbook of fluid dynamics (Washington, DC : CRC Press)

Johnson C G, Kokelaar B P, Iverson R M, Logan M, LaHusen R G and Gray J M N T 2012 Grain-size segregation and levee formation in geophysical mass flows J. Geophys. Res. 117 F01032

Johnson B C, Campbell C S, Melosh H J 2016 The reduction of friction in long runout landslides as an emergent phenomenon J. Geophys. Res. Earth Surf. in press

Johnsson A, Reiss D, Hauber E, Hiesinger H and Zanetti M 2014 Evidence for very recent melt-water and debris flow activity in gullies in a young mid-latitude crater on Mars Icarus 235 37-54

Jop P, Forterre Y, and Pouliquen O 2005 Crucial role of sidewalls in granular surface flows: consequences for the rheology Journal of Fluid Mechanics 541 167-192

Jop P, Forterre Y and Pouliquen O 2006 A constitutive law for dense granular flows Nature 441 727-730

Jop P 2015 Rheological properties of dense granular flows C. R. Physique 16 62-72

Jouannic G, Gargani J, Costard F, Ori G G, Marmo C, Schmidt F and Lucas A 2012 Morphological and mechanical characterization of past and present flow activities in a periglacial environment: the case of the Russell dune (Mars) Planetary and Space Science 71 38-54

Kaitna R, Dietrich W E, Hsu L 2014 Surface slopes, velocity profiles and fluid pressure in coarse-grained debris flows saturated with water and mud Journal of Fluid Mechanics 741 277-403

Kanamori H and Given J W 1982 Analysis of long-period seismic waves excited by the May 18, 1980, eruption of Mount St. Helens - a terrestrial monopole J. Geophys. Res. 87 5422-5432

Kawakatsu H 1989 Centroid single force inversion of seismic-waves generated by landslides J. Geophys. Res. 94 12363-12374

Kawamura K, Laberg J S and Kanamatsu T 2014 Potential tsunamigenic submarine landslides in active margins Marine Geology 356 44-49

Kean J W, McCoy S W, Tucker G E, Staley D M and Coe J A 2013 Runoff-generated debris flows: Observations and modeling of surge initiation, magnitude, and frequency J. Geophys. Res.-Earth Surface 118 2190-2207

Kelfoun K and Druitt T H 2005 Numerical modeling of the emplacement of Socompa rock avalanche, Chile $J$. Geophys. Res. 110 B12202

Kerswell R R 2005 Dam break with Coulomb friction: A model for granular slumping? Physics of Fluids 17 057101

Kiesgen de Richter S, Le Caër G and Delannay R 2012 Dynamics of rearrangements during inclination of granular packings: the avalanche precursor regime Journal of Statistical Mechanics: Theory and Experiment 04 P04013.

Kokelaar B P, Graham R L, Gray J M N T and Vallance J W 2014 Fine-grained linings of leveed channels facilitate runout of granular flows Earth and Planetary Science Letters 385 172-180

Kumaran V and Maheshwari S 2012 Transition due to base roughness in a dense granular flow down an inclined plane Phys. Fluids 24, 053302

Kumaran V and Bharathraj S 2013 The effect of base roughness on the development of a dense granular flow down an inclined plane Phys. Fluids 25070604

Kumaran V 2015 Kinetic theory for sheared granular flows C. R. Physique 16 51-61

Lacaze L, Phillips J C and Kerswell R R 2008 Planar collapse of a granular column: experiments and discrete element simulations Physics of Fluids 20063302

Lacaze L and Kerswell R R 2009 Axisymmetric granular collapse: A transient 3D flow test of viscoplasticity Phys. Rev. Lett. 102108305

Lacroix P, Grasso J-R, Roulle J, Giraud G, Goetz D, Morin S and Helmstetter A 2012 Monitoring of snow avalanches using a seismic array: Location, speed estimation, and relationships to meteorological variables J. Geophys. Res. 117 F01034

Lagrée P-Y, Staron L and Popinet S 2011 The granular column collapse as a continuum: validity of a twodimensional Navier-Stokes model with a $\square$ (I)-rheology J. Fluid Mech. 686 378-408 
Lajeunesse E, Mangeney-Castelnau A and Vilotte J P 2004 Spreading of a granular mass on a horizontal plane Physics of Fluids $\mathbf{1 6}$ 2371-2381

Lajeunesse E, Monnier J B and Homsy G M 2005 Granular slumping on a horizontal surface Physics of Fluids 17103302

Lajeunesse E, Quantin C, Allemand P and Delacourt C 2006 New insights on the runout of large landslides in the Valles-Marineris canyons, Mars Geophysical Research Letters 33 L04403

Larcher M, Fraccarollo L, Armanini A and Capart H 2007 Journal Hydr. Res. 45 59-71

La Rocca M, Galluzzo D, Saccorotti G, Tinti S, Cimini G B and Pezzo E D 2004 Seismic signals associated with landslides and with a tsunami at Stromboli Volcano, Italy Bulletin of the Seismological Society of America 94 1850-1867

Le Friant A, Heinrich P, Deplus C and Boudon G 2003 Numerical simulation of the last flank collapse event of Montagne Pelée, Martinique, Lesser Antilles Geophys. Res. Lett. 301034

Le Friant A, Harford C L, Deplus C, Boudon G, Sparks R S J, Herd R A and Komorowski J C 2004 Geomorphological evolution of Montserrat (West Indies): importance of flank collapse and erosional processes Journal of the Geological Society, London 161 147-160

Le Friant A et al. 2010 Eruption of Soufrière Hills (1995-2009) from an offshore perspective: insights from repeated swath bathymetry surveys Geophys. Res. Lett. 37 L11307

Legros F, Cantagrel J M and Devouard B 2000 Pseudotachylyte (frictionite) at the base of the Arequipa volcanic land slide deposit (Peru): implications for emplacement mechanisms J. Geol. 108 601-611

Legros F 2002 The mobility of long-runout landslides Engineering Geology 63301 - 331

Levy C, Mangeney A, Bonilla F, Hibert C, Calder E and Smith P J 2015 Friction weakening in granular flows deduced from seismic records at the Soufrière Hills Volcano, Montserrat J. Geophys. Res. 120 7536-7557

Lin C H, Kumagai H, Ando M and Shin T. C 2010 Detection of landslides and submarine slumps using broadband seismic networks J. Geophys. Res. 37 L22309

Louge M Y and Keast S C 2001 On dense granular flows down flat frictional inclines Physics of Fluids 13 1213-1233

Louge M Y, Valance A, Lancelot P, Delannay R and Artieres O 2015 Granular flows on a dissipative base Phys. Rev. E under review

Lube G, Huppert H E, Sparks R S J and Hallworth M A 2004 Axisymmetric collapse of granular columns Journal of Fluid Mechanics Lube G, Huppert H E175-199

Lube G, Huppert H E, Sparks R S J and Freundt A 2005 Collapses of two dimensional granular columns Phys. Rev. E 72041301

Lube G, Huppert H E, Sparks R S J and Freundt A 2007 Static and flowing regions in granular collapses down channels Physics of Fluids 19043301

Lube G, Huppert HE, Sparks RSJ and Freundt A 2011 Granular column collapses down rough, inclined channels Journal of Fluid Mechanics 675 347-368

Lube G, Cronin S J, Manville V, Procter J N, Cole S E and Freundt A 2012 Energy growth in laharic mass flows Geology 40 475-478

Lucas A and Mangeney A 2007 Mobility and topographic effects for large Valles Marineris landslides on Mars Geophys. Res. Lett. 34 L10201

Lucas A 2010 Dynamique des instabilités gravitaires par modélisation et télédétection: Application aux exemples martiens Ph.D. thesis IPGP Paris

Lucas A, Mangeney A, Mège D and Bouchut F 2011 Influence of the scar geometry on landslide dynamics and deposits: Application to Martian landslides J. Geophys. Res. - Planets 116 E10001

Lucas A, Mangeney A and Ampuero J P 2014 Frictional weakening in landslides on Earth and on other planetary bodies Nature Communication 53417

Lusso C, Bouchut F, Ern A and Mangeney A 2015a A simplified model of thin layer static/flowing dynamics for granular materials with yield Preprint hal-00992309v2

Lusso C, Ern A, Bouchut F, Mangeney A, Farin M and Roche O 2015b Two-dimensional simulation by regularization of free surface viscoplastic flows with Drucker-Prager yield stress and application to granular collapse Preprint hal-01133786v1

Major J J and Iverson R M 1999 Debris-flow deposition: effects of pore-fluid pressure and friction concentrated at flow margins Geological Society of America Bulletin 111 1424-1434.

Malin M C and Edgett K S 2000 Evidence for recent groundwater seepage and surface runoff on Mars Science 228 2330-2335

Malloggi F, Lanuza J, Andreotti B and Clément E 2006 Erosion waves: Transverse instabilities and fingering Europhysics Letters 75825

Manga M, Patel A and Dufek J 2011 Rounding of pumice clasts during transport: filed measurements and laboratory studies Bulletin of Volcanology 73 321-333 
Mangeney-Castelnau A, Vilotte J-P, Bristeau M O, Perthame B, Bouchut F, Simeoni C and Yerneni S 2003 Numerical modeling of avalanches based on Saint Venant equations using a kinetic scheme Journal of Geophysical Research 1082527

Mangeney-Castelnau A, Bouchut F, Vilotte J-P, Lajeunesse E, Aubertin A and Pirulli M 2005 On the use of Saint Venant equations to simulate the spreading of a granular mass Journal of Geophysical Research 110 B09103

Mangeney A, Bouchut F, Thomas N, Vilotte J-P and Bristeau M O 2007a Numerical modeling of selfchanneling granular flows and of their levee-channel deposit Journal of Geophysical Research 112 F02017

Mangeney A, Tsimring L S, Volfson D, Aranson I S and Bouchut F 2007b Avalanche mobility induced by the presence of an erodible bed and associated entrainment Geophys. Res. Lett. 34 L22401

Mangeney A, Roche O, Hungr O, Mangold N, Faccanoni G and Lucas A 2010 Erosion and mobility in granular collapse over sloping beds Journal of Geophysical Research 115 F03040

Mangeney A 2011 Geomorphology: Landslide boost from entrainment Nature Geoscience 4 77-78

Mangold N, Costard F and Forget F 2003 Debris flows over sand dunes on Mars: Evidence for liquid water $J$. Geophys. Res. 1085027.

Mangold N, Mangeney A, Migeon V, Ansan V, Lucas A, Baratoux D and Bouchut F 2010 Sinuous gullies on Mars: Frequency, distribution, and implications for flow properties J. Geophys. Res.- Planets 115 E11001

Marchi L, Arattano M and Deganutti A M 2002 Ten years of debris-flow monitoring in the Moscardo Torrent (Italian Alps) Geomorphology 46 1-17

Martin N, Ionescu I , Mangeney A and Farin M 2015 Continuum viscoplastic simulation of a granular column collapse on large slopes : $\mu(I)$ rheology and lateral wall effects submitted

Masson D G, Harbitz C B, Wynn R B, Pedersen G and Løvholt F 2006 Submarine landslides: processes, triggers and hazard prediction Phil. Trans. R. Soc. A 364 2009-2039

McArdell B W, Bartelt P and Kowalski J 2007 Field observations of basal forces and fluid pore pressure in a debris flow Geophysical Research Letters 34 L07406

McCoy S W, Kean J W, Coe J A, Tucker G E and Staley D M 2012 Sediment entrainment by debris flows: In situ measurments from the headwaters of a steep catchment Journal of Geophysical Research 117 F03016

McCoy S W, Tucker G E, Kean J W and Coe J A 2013 Field measurement of basal forces generated by erosive debris flows Journal of Geophysical Research - Earth Surface 118 589-602

McDougall S and Hungr O 2004 A model for the analysis of rapid landslide motion across three-dimensional terrain Canadian Geotechnical Journal 41 1084-1097

McElwaine J N and Turnbull B 2005 Air pressure data from the Vallée de la Sionne avalanches of 2004 Journal of Geophysical Research 110 F03010

McElwaine J N, Takagi D and Huppert H E 2012 Surface curvature of steady granular flows Granular Matter 14 229-234

McEwen A S 1989 Mobility of large rock avalanches: Evidence from Valles Marineris, Mars Geology 17 11111114

Meiburg E and Kneller B 2010 Turbidity currents and their deposits Annual Review of Fluid Mechanics 42 135156

Mériaux C 2006 Two dimensional fall of granular columns controlled by slow horizontal withdrawal of a retaining wall Physics of Fluids 18093301

Mériaux C and Triantafiliou T 2008 Scaling the final deposits of dry cohesive granular columns after collapse and quasi-static fall Physics of Fluids 20033301

Meruane C, Tamburrino A and Roche O 2012 Dynamics of dense granular flows of small-and-large-grains mixtures in an ambient fluid Physical Review E 86026311

Meunier P, Hovius N and Haines A J 2007 Regional patterns of earthquake-triggered landslides and their relation to ground motion Geophys. Res. Lett. 34 L20408

Montgomery D R, Schmidt K M, Dietrich W E and McKean J 2009 Instrumental record of debris flow initiation during natural rainfall: Implications for modeling slope stability Journal of Geophysical Research 114 F01031

Montserrat S, Tamburrino A, Roche O and Niño Y 2012 Pore fluid pressure diffusion in defluidizing granular columns Journal of Geophysical Research-Earth Surface 117 F02034

Moretti L, Mangeney A, Capdeville Y, Stutzmann E, Huggel C, Schneider D and Bouchut F 2012 Numerical modeling of the Mount Steller landslide flow history and of the generated long period seismic waves Geophys. Res. Lett. 39 L16402

Moretti L, Allstadt K, Mangeney A, Capdeville Y, Stutzmann E and Bouchut F 2015 Numerical modeling of the Mount Meager landslide constrained by its force history derived from seismic data J. Geophys. Res. Solid Earth 120 2579-2599

Nichol K, Zanin A, Bastien R, Wandersman E and van Hecke M 2010 Flow-Induced Agitations Create a Granular Fluid Phys. Rev. Lett. 104078302 
Nishimura K, Sandersen F, Kristensen K and Lied K 1995 Measurements of powder snow avalanche Surveys in Geophysics 16 649-660

Nishimura K and Ito Y 1997 Velocity distribution in snow avalanches Journal of Geophysical Research 112 27297-27303

Normark W R, Posamentier H and Mutti E 1993 Turbidite systems: state of the art and future directions Reviews of Geophysics 31 91-116

Pailha M, Nicolas M and Pouliquen O 2008 Initiation of underwater granular avalanches: influence of the initial volume fraction Phys. Fluids 2011701

Pailha M and Pouliquen O 2009 A two-phase flow description of the initiation of underwater granular avalanches J. Fluid Mech. 633 115-135

Pelanti M, Bouchut F and Mangeney A 2008 A Roe-Type Scheme for Two-Phase Shallow Granular Flows over Variable Topography Math. Model. Num. Analy. (ESAIM :M2AN) 42 851-885

Phillips J C, Hogg A J, Kerswell R R and Thomas N H 2006 Enhanced mobility of granular mixtures of fine and coarse particles Earth and Planetary Science Letters 246 466-480

Pierson T 1986 Flow behavior of channelized debris flows, Mount St. Helens, Washington Hillslope Processes ed. A. D. Abrahams (Boston, Massachusets: Allen and Unwin) pp 269-296

Piper D J W, Shor A N, Farre J A, O'Connell S and Jacobi R 1985 Sediment slides and turbidity currents on the Laurentian Fan: sidescan sonar investigations near the epicenter of the 1929 Grand Banks earthquake Geology 13 528-541

Pirulli M, Bristeau M O, Mangeney A and Scavia C 2007 The effect of the earth pressure coefficients on the runout of granular material Environmental modelling \& software 22 1437-1454

Pirulli M and Sorbino G 2008 Assessing potential debris flow runout: a comparison of two simulation models Natural Hazards and Earth System Sciences 8 961-971

Pirulli M and Mangeney A 2008 Results of back-analysis of the propagation of rock avalanches as a function of the assumed rheology Rock Mechanics and Rock Engineering 41 59-84

Pitman E B and Lee L 2005 A two-fluid model for avalanche and debris flows Phil. Trans. R. Soc. A 3631573 1601

Pittari A and Cas R A F 2004 Sole marks at the base of the late Pleistocene Abrigo Ignimbrite, Tenerife: implications for transport and depositional processes at the base of pyroclastic flows. Bulletin of Volcanology 66 356-363

Pouliquen O, Delour J and Savage S B 1997 Fingering in granular flows Nature 386 816-81

Pouliquen O 1999 Scaling laws in granular flows down rough inclined planes Physics of Fluids 11 542-548

Pouliquen O and Vallance J W 1999 Segregation induced instabilities of granular fronts Chaos 9 621-630

Pouliquen O and Forterre Y 2002 Friction law for dense granular flows: application to the motion of a mass down a rough inclined plane Journal of fluid mechanics 453 133-151

Pouliquen O, Cassar C, Forterre Y, Jop P and Nicolas M 2005 How do grains flow: towards a simple rheology for dense granular flows Proc. Powder and Grains 2005 859-865

Pudasaini S P, Hutter K, Hsiau S-S, Tai S-C, Wang Y and Katzenbach 2007 Rapid flow of dry granular materials down inclined chutes impinging on rigid walls Physics of Fluids, AIP 19, 053302

Quantin C, Allemand P and Delacourt C 2004 Morphology and geometry of Valles Marineris landslides Planet. Space Sci. 52 1011-1022

Rajchenbach J 2003 Dense, Rapid Flows of Inelastic Grains under Gravity Phys. Rev. Lett. 90144302

Rondon L, Pouliquen O and Aussillous P 2011 Granular collapse in a fluid: role of the initial volume fraction Phys. Fluids 23073301

Rastello M, Rastello F, Bellot H, Ousset F, Dufour F and Meier L 2011 Size of snow particles in a powder-snow avalanche Journal of Glaciology 57 151-156

Reddy K A, Forterre Y and Pouliquen O 2011 Evidence of Mechanically Activated Processes in Slow Granular Flows Phys. Rev. Lett. 106108301

Reynolds 1886 Dilatancy Nature 33 429-430

Richard P, Valance A, Métayer J-F, Sanchez P, Louge M and Delannay R 2008 Rheology of Confined Granular Flows: Scale Invariance, Glass Transition, and Friction Weakening Physical Review Letters 101248002

Richardson J F and Zacki W N 1954 Sedimentation and fluidization: part 1. Trans. Inst. Chem. Eng. 32 35-53

Richman M 1988 Boundary conditions based on a modified Maxwellian velocity distribution for flows of identical, smooth, nearly elastic spheres. Acta Mech. 75 227-240

Richman M W and Marciniec R P 1990 Gravity-driven granular fl ows of smooth, inelastic spheres down bumpy inclines J. Appl. Mech. 57 1036-1043

Ripepe M, De Angelis S, Lacanna G, Poggi P, Williams C, Marchetti E, Delle Donne D and Ulivieri G 2009 Tracking pyroclastic flows at Soufrière Hills volcano EOS Transactions 90 229-236

Roche O, Gilbertson M A, Phillips J C and Sparks R S J 2005 Inviscid behaviour of fines-rich pyroclastic flows inferred from experiments on gas-particle mixtures Earth and Planetary Science Letters 240 401-414 
Roche O, Montserrat S, Niño Y and Tamburrino A 2008 Experimental observations of water-like behavior of initially fluidized, dam break granular flows and their relevance for the propagation of ash-rich pyroclastic flows Journal of Geophysical Research 113 B12203

Roche O, Atalli M, Mangeney A and Lucas A 2011 On the run-out distance of geophysical gravitational flows: insight from fluidized granular collapse experiments Earth and Planetary Science Letters 311 375-385

Roche O. 2012 Depositional processes and gas pore pressure in pyroclastic flows: an experimental perspective Bulletin of Volcanology 74 1807-1820

Roche O, Phillips J C and Kelfoun K 2013a Pyroclastic density currents Modeling Volcanic Processes: The Physics and Mathematics of Volcanism ed Fagents S A, Gregg T K P, Lopes R M C (Cambridge University Press) pp 203-229

Roche O, Niño Y, Mangeney A, Brand B, Pollock N and Valentine GA 2013b Dynamic pore-pressure variations induce substrate erosion by pyroclastic flows Geology 41 1107-1110

Roche O, Buesch D C and Valentine G A 2016 Slow-moving and far-travelled dense pyroclastic flows during the Peach Spring super-eruption Nat. Comm. 710890

Roux S and Radjai F 1998 Texture-dependent rigid plastic behavior (Kluwer)

Rowley P J 2011 Shear-derived mixing in dense granular flows Journal of Sedimentary Research 81 974-884

Savage S B 1979 Gravity flow of cohesionless granular materials in chutes and channels J. Fluid Mech. 92 53-96

Savage S B and Lun C K K 1988 Particle size segregation in inclined chute flow of dry cohesionless granular solids Journal of Fluid Mechanics 189 311- 335

Savage S B and Hutter K 1989 The motion of a finite mass of granular material down a rough incline Journal of Fluid Mechanics 199 177-215

Schneider D, Bartelt P, Caplan-Auerbach J, Christen M, Huggel C and McArdell B W 2010 Insights into rockice avalanche dynamics by combined analysis of seismic recordings and a numerical avalanche model $J$. Geophys. Res. 115 F04026

Schneider D, Kaitna R, Dietrich W E, Hsu L, Huggel C, McArdell B W 2011 Frictional behavior of granular gravel-ice mixtures in vertically rotating drum experiments and implications for rock-ice avalanches, Cold Reg. Sci. Technol. 69 (1) 70-90

Schweizer J, Jamieson J B and Schneebeli M 2003 Snow avalanche formation Reviews of Geophysics 411016

Siavoshi S and Kudrolli A 2005 Failure of a granular step Phys. Rev. E 71051302

Silbert L E, Ertas D, Grest G S, Halsey T C, Levine D and Plimpton S J 2001 Granular flow down an inclined plane: Bagnold scaling and rheology Phys. Rev. E 64051302

Silbert L E, Grest G S, Plimpton S J and Levine D 2002 Boundary effects and self-organization in dense granular flows Phys. Fluids 14, 2637

Sovilla B, Burlando P and Bartelt P 2006 Field experiments and numerical modeling of mass entrainment in snow avalanches Journal of Geophysical Research 111 F03007

Sovilla B, McElwaine J N, Schaer M and Vallet J 2010 Variation of deposition depth with slope angle in snow avalanches: Measurements from Vallée de la Sionne Journal of Geophysical Research: Earth Surface 115 F02016

Sparks R S J, Bonnecaze R T, Huppert H E, Lister J R, Hallworth M A, Mader H and Phillips J 1993 Sedimentladen gravity currents with reversing buoyancy Earth and Planetary Science Letters 114 243-257

Sparks R, Gardeweg M, Calder E and Matthews S 1997 Erosion by pyroclastic flows on Lascar Volcano, Chile Bull. Volcanol. 58 557-565

Staron L and Hinch E J 2005 Study of the collapse of granular columns using 2D discrete-grains simulation Journal of Fluid Mechanics 545, 1-27

Staron L and Hinch E J 2007 The spreading of a granular mass: role of grain properties and initial conditions Granular Matter 9 205-217

Staron L, Lagrée P Y, Josserand C and Lhuillier D 2010 Flow and jamming of a two-dimensional granular bed: Toward a nonlocal rheology? Physics of Fluids 22113303

Tai Y C, Gray J M N T, Hutter K and Noelle S 2001 Flow of dense avalanches past obstructions annals of glaciology 32 281-284

Taberlet N, Richard P, Valance A, Losert W, Pasini J M, Jenkins J T and Delannay R 2003 Superstable Granular Heap in a Thin Channel Phys. Rev. Lett. 91264301

Taberlet N, Richard P, Henry E and Delannay R 2004 The growth of a Super Stable Heap: An experimental and numerical study Europhysics Letters 68 515-521

Taberlet N, Richard P and Delannay R 2008 The effect of sidewall friction on dense granular flows Computers \& Mathematics with Applications 55230 - 234

Takagi D, McElwaine J N and Huppert H E 2011 Shallow granular flows Phys. Rev. E 83031306

Takahashi T 1991 Debris flow (Rotterdam: Balkema)

Thompson E L and Huppert H E 2007 Granular column collapses: further experimental results Journal of Fluid Mechanics 575 177-186 
Tripathi A and Khakhar D V 2011 Rheology of binary granular mixtures in the dense flow regime Physics of Fluids 23113302

Turnbull B and McElwaine J N 2007 A comparison of powder snow avalanches at Vallée de la Sionne with plume theories Journal of Glaciology 53 30-40

Valentine G A 1987 Stratified flow in pyroclastic surges. Bulletin of Volcanology 49 616-630

Valverde J M and Soria-Hoyo C 2015 Vibration-induced dynamical weakening of pyroclastic flows: Insights from rotating drum experiments Journal of Geophysical Research, Solid Earth 120 6182-6190

van der Vaart K, Gajjar P, Epely-Chauvin G, Andreini N, Gray J M N T and Ancey C 2015 Underlying Asymmetry within Particle Size Segregation Physical Review Letters 114238001

Vreman A W, Al-Tarazi M, Kuipers J A M, Van Sint Annaland M and Bokhove O 2007 Supercritical shallow granular flow through a contraction: experiment, theory and simulation J. Fluid Mechanics 578 233-269

Vilajosana I, Khazaradze G, Suriñach E, Lied E and Kristensen K 2007 Snow avalanche speed determination using seismic methods Cold regions science and technology 49 2-10

Voellmy A 1955 Über die Zerstörungskraft von Lawinen Tech. rep., Schweiz. Bauzeitung 73

Vriend N M, McElwaine J N, Sovilla B, Keylock C J, Ash M and Brennan P V 2013 High Resolution Radar Measurements of Snow Avalanches Geophysical Research Letters 40 727-731

Weidinger J T, Korup O, Munack H, Altenberger U, Dunning S A, Tippelt G and Lottermosere W 2014 Giant rock slides from the inside Earth and Planetary Science Letters 389 62-73

Wiederseiner S, Andreini N, Épely-Chauvin G, Moser G, Monnereau M, Gray J M N T and Ancey C 2011 Experimental investigation into segregating granular flows down chutes Physics of Fluids 23013301

Woodhouse M J, Thornton A R, Johnson C G, Kokelaar B P and Gray J M N T 2012 Segregation-induced fingering instabilities in granular free-surface flows Journal of Fluid Mechanics 709 543-580

Woods A and Wohletz K 1991 Dimensions and dynamics of co-ignimbrite eruption columns Nature 350225 227

Xu H, Louge M and Reeves A 2003 Solutions of the kinetic theory for bounded collisional granular flows Continuum Mech. Thermodyn. 15 321-349

Yamada M, Matsushi Y, Chigira M and Mori J 2012 Seismic recordings of landslides caused by Typhoon Talas (2011), Japan Geophys. Res. Lett. 39 L13301

Yamada M, Mangeney A, Matsushi Y and Moretti L 2015 Estimation of dynamic friction process of the Akatani landslide based on the waveform inversion and numerical simulation Geophys. Res. Lett. submitted

Yang F L and Hunt M L 2006 Dynamics of particle-particle collisions in a viscous liquid Physics of Fluids 18 121506.

Zanuttigh B and Lamberti A 2007 Instability and surge development in debris flows Reviews of Geophysics 45 RG3006

Zanuttigh B and Ghilardi P 2010 Segregation process of water-granular mixtures released down a steep chute $J$. Hydrology 391 175-187

Zhao J, Moretti L, Mangeney A, Stutzmann E, Kanamori H, Capdeville Y, Calder E S, Hibert C, Smith P J, Cole $\mathrm{P}$ and LeFriant A 2015 Model space exploration for determining landslide source history from long period seismic data Pure Appl. Geophys. 172 389-413 